\title{
The Evolutionary Status of Protostellar Clumps Hosting Class II Methanol Masers
}

DOI:

10.1093/mnras/staa233

\section{Document Version}

Accepted author manuscript

Link to publication record in Manchester Research Explorer

\section{Citation for published version (APA):}

Jones, B. M., Fuller, G. A., Breen, S. L., Avison, A., Green, J. A., Traficante, A., Elia, D., \{Ellingsen\}, S. P., Voronkov, M. A., Merello, M., Molinari, S., \& Schisano, E. (2020). The Evolutionary Status of Protostellar Clumps Hosting Class II Methanol Masers. Monthly Notices of the Royal Astronomical Society. https://doi.org/10.1093/mnras/staa233

Published in:

Monthly Notices of the Royal Astronomical Society

\section{Citing this paper}

Please note that where the full-text provided on Manchester Research Explorer is the Author Accepted Manuscript or Proof version this may differ from the final Published version. If citing, it is advised that you check and use the publisher's definitive version.

\section{General rights}

Copyright and moral rights for the publications made accessible in the Research Explorer are retained by the authors and/or other copyright owners and it is a condition of accessing publications that users recognise and abide by the legal requirements associated with these rights.

\section{Takedown policy}

If you believe that this document breaches copyright please refer to the University of Manchester's Takedown Procedures [http://man.ac.uk/04Y6Bo] or contact uml.scholarlycommunications@manchester.ac.uk providing relevant details, so we can investigate your claim.

\section{OPEN ACCESS}




\title{
The Evolutionary Status of Protostellar Clumps Hosting Class II Methanol Masers
}

B. M. Jones ${ }^{1}{ }^{\star}$ G. A. Fuller ${ }^{1,2}$, S. L. Breen ${ }^{3}$, A. Avison ${ }^{1,2}$, J. A. Green ${ }^{4}$, A. Traficante ${ }^{5}$, D. Elia ${ }^{5}$, S. P. Ellingsen ${ }^{6}$, M. A. Voronkov ${ }^{7}$, M. Merello ${ }^{5,8}$, S. Molinari ${ }^{5}$, E. Schisano .

${ }^{1}$ Jodrell Bank Centre for Astrophysics, Department of Physics and Astronomy, The School of Natural Sciences, The University of Manchester, Manchester M13 9PL, UK

${ }^{2}$ UK ALMA Regional Centre Node, M13 9PL, UK

${ }^{3}$ Sydney Institute for Astronomy (SIfA), School of Physics, University of Sydney, NSW 2006, Australia

${ }^{4}$ CSIRO Astronomy and Space Science, 26 Dick Perry Avenue, Kensington, WA 6151, Australia

${ }^{5}$ IAPS-INAF, Via Fosso del Cavaliere 100, 00133 Rome, Italy

${ }^{6}$ School of Natural Sciences, University of Tasmania, Private Bag 37, Hobart, Tasmania 7001, Australia

${ }^{7}$ CSIRO Astronomy and Space Science, Australia Telescope National Facility, Box 76, Epping, NSW 1710, Australia

${ }^{8}$ Universidade de São Paulo, IAG Rua do Matão, 1226, Cidade Universitária, 05508-090, São Paulo, Brazil

Accepted XXX. Received YYY; in original form ZZZ

\begin{abstract}
The Methanol MultiBeam survey (MMB) provides the most complete sample of Galactic massive young stellar objects (MYSOs) hosting $6.7 \mathrm{GHz}$ class II methanol masers. We characterise the properties of these maser sources using dust emission detected by the Herschel Infrared Galactic Plane Survey (Hi-GAL) to assess their evolutionary state. Associating 731 (73\%) of MMB sources with compact emission at four Hi-GAL wavelengths, we derive clump properties and define the requirements of a MYSO to host a $6.7 \mathrm{GHz}$ maser. The median far-infrared (FIR) mass and luminosity are $630 \mathrm{M}_{\odot}$ and $2500 \mathrm{~L}_{\odot}$ for sources on the near side of Galactic centre and $3200 \mathrm{M}_{\odot}$ and $10000 \mathrm{~L}_{\odot}$ for more distant sources. The median luminosity-to-mass ratio is similar for both at $\sim 4.2 \mathrm{~L}_{\odot} / \mathrm{M}_{\odot}$. We identify an apparent minimum $70 \mu \mathrm{m}$ luminosity required to sustain a methanol maser of a given luminosity (with $L_{70} \propto L_{6.7}{ }^{0.6}$ ). The maser host clumps have higher mass and higher FIR luminosities than the general Galactic population of protostellar MYSOs. Using principal component analysis, we find 896 protostellar clumps satisfy the requirements to host a methanol maser but lack a detection in the MMB. Finding a $70 \mu \mathrm{m}$ flux density deficiency in these objects, we favour the scenario in which these objects are evolved beyond the age where a luminous $6.7 \mathrm{GHz}$ maser can be sustained. Finally, segregation by association with secondary maser species identifies evolutionary differences within the population of $6.7 \mathrm{GHz}$ sources.
\end{abstract}

Key words: masers - stars: formation - stars: massive

\section{INTRODUCTION}

The formation of massive stars within our galaxy is currently a poorly constrained process relative to our understanding of the mechanisms which produce stars of a similar mass to the Sun (Krumholz 2014). For stars of $>8 \mathrm{M}_{\odot}$, the onset of fusion in a protostellar core before it has finished accreting material from its surroundings leads to a complicated interplay between the strong outwards feedback and the in-

^ E-mail: bethany.jones@manchester.ac.uk (BMJ) falling material (Kudritzki 2002; Zinnecker \& Yorke 2007). Further complexity is added by the tendency for parsec-scale clumps within molecular clouds to form clusters of stars with a range of masses, rather than isolated massive stars (Lada \& Lada 2003). The evolution of a single, rare, massive protostar is therefore difficult to follow as the accretion of mass and subsequent evolution may be influenced by other cluster members. The feedback from multiple high-mass protostars also rapidly processes their natal cloud, both kinematically and chemically, to quickly erase any of the initial conditions of the environments in which they form. 
Although a framework comparable to the Class $0 / \mathrm{I} / \mathrm{II} / \mathrm{III}$ classification of low-mass protostars is currently lacking in the high-mass regime, several distinct evolutionary phases of a young high-mass protostar have been identified (e.g. Zinnecker \& Yorke 2007; Svoboda et al. 2016). This includes the formation of cold starless (or very young protostellar) clumps (e.g. Traficante et al. 2017), hot cores (e.g. Cesaroni 2005) and finally Hiı regions (e.g. Kurtz 2005). To fully understand the mechanisms governing the evolution of massive young stellar objects (MYSOs), snapshots along the entire evolutionary path are required. Due to the relatively short timescales between the onset of fusion and the dispersal of the parent cloud, the point at which an MYSO attains its final mass is not well characterised. So far, the deeply embedded nature of MYSOs within the star forming clumps makes it challenging to identify a population of sources at this point in evolution, often requiring interferometric resolutions to probe cores of $\sim 0.1 \mathrm{pc}$ size forming individual stars (Tan et al. 2014).

Aside from directly observing the emission from a protostar itself, other features of high-mass star forming regions have been tied to the evolutionary status of protostellar objects. Examples of this include extended green emission (EGOs), which previous authors have found to be associated with MYSOs in the earliest stages of evolution with ongoing outflow activity (e.g. Cyganowski et al. 2008; Chen et al. 2013), and various maser species (Forster \& Caswell 1989; Ellingsen et al. 2007; Breen et al. 2010). Found only in the hot, dusty inner circumstellar environments of highmass protostars, class II methanol masers allow us to isolate individual sources within a clump and have been proposed as a marker of a particular, although poorly constrained, stage of massive star formation that is expected to last between $2.5 \times 10^{4}$ and $4.5 \times 10^{4}$ years (van der Walt 2005).

Class II methanol masers are found in MYSOs prior to the destruction of methanol within the immediate environment of the protostar and emit strongly at a main line frequency of $6.7 \mathrm{GHz}$, (Minier et al. 2003; Ellingsen 2006). Unlike class I methanol masers which are collisionally pumped, class II methanol masers are radiatively pumped by strong infrared radiation at $\sim 70 \mu \mathrm{m}$ (Cragg et al. 1992). Sufficient flux at this wavelength is provided by the thermal reemission of the strong UV emission of a massive protostar by surrounding dust (Breen et al. 2013). In addition to the main $6.7 \mathrm{GHz}$ line, masing of a second methanol line at $12.2 \mathrm{GHz}$ also occurs under similar physical conditions (Cragg et al. 2001). The focus of this paper is to comprehensively characterise the Galactic population of MYSOs hosting class II methanol masers, so that the evolutionary status of these sources can be constrained.

Other maser species in a clump may also trace various evolutionary stages of a host protostar. Collisional masers such as water and class I methanol masers are often associated with the shocks from molecular outflows (Slysh et al. 1994; Walsh et al. 2011; Cyganowski et al. 2009), both in low and high-mass star formation, but are also found in other environments such as evolved stars (Deacon et al. 2007). Hydroxyl masers are found outside HiI regions including those created as the protostar begins to ionize its surrounding in the late stages of its evolution. Observations of excited-state hydroxyl masers can also be used to measure the magnetic field in star forming regions through their hyperfine split- ting (Caswell \& Vaile 1995; Caswell 2003, Avison et al. in prep.). Unlike the $6.7 \mathrm{GHz}$ methanol masers, each of these maser species may be diagnostic of a range of astrophysical scenarios that share common physical conditions, and can trace multiple stages of evolution even when associated with an MYSO. Class II methanol masers are therefore the most appropriate for isolating a population of protostars in a given evolutionary state, and additional masers can offer further insight into the characteristics of a massive protostar.

Catalogues from large surveys of Galactic masers have recently been released, with the Methanol MultiBeam Survey (MMB) providing a complete sample of luminous 6.7 GHz class II methanol masers for the southern Galactic plane (Green et al. 2009). Only observed as a secondary line to $6.7 \mathrm{GHz}$, the targeted follow-up study of all MMB sources to detect the second class II methanol line at $12.2 \mathrm{GHz}$ has also been completed (Breen et al. 2016). An untargeted survey of hydroxyl masers for the inner Galactic plane is provided by Southern Parkes Large-Area Survey in Hydroxyl (SPLASH, Dawson et al. 2014), and the rarer excitedstate hydroxyl (ex-OH) masers were co-observed during the MMB. Avison et al. (2016) released a catalogue of the ex$\mathrm{OH}$ masers for the entire MMB survey range, with their magnetic field properties studied further in Avison et al. (in prep.). An untargeted survey of the $22 \mathrm{GHz}$ maser line of water has also been observed by the $\mathrm{H}_{2} \mathrm{O}$ Southern Galactic Plane Survey (HOPS, Walsh et al. 2011) for the inner Galactic Plane, giving a large sample of water maser detections to supplement the higher sensitivity follow-up towards a small number of MMB sources by Titmarsh et al. (2014, 2016). Breen et al. (2018) recently investigated the Galactic populations of these masers and the associations between them, finding further evidence to support an evolutionary sequence for the common maser species found in the vicinity of young stars.

To complement the large coverage of the maser studies, the Herschel Infrared Galactic Plane Survey (Hi-GAL) has mapped the entire Galactic plane at high-resolution in the far-infrared (Molinari et al. 2010b,a). Peaking in this wavelength regime and visible over several of the 5 wavelengths observed, the thermal dust emission from MYSOs visible as compact objects with Hi-GAL can be used to reconstruct the spectral energy distribution (SED) and derive properties for each source such as temperature and mass surface density, in addition to luminosity and mass if the distance to a source is known. Counterparts at wavelengths $\leq 70 \mu \mathrm{m}$ may also be used as indicators that a clump is no longer starless but hosts at least one protostar (Dunham et al. 2008). In addition to the $70 \mu \mathrm{m}$ coverage of Hi-GAL, compact source catalogues for surveys in the mid-infrared (MIR), such as the GLIMPSE (Benjamin et al. 2003) and MIPSGAL (Carey et al. 2009) surveys with the Spitzer (Churchwell et al. 2009) satellite, are now available for the inner Galactic plane. Previous studies have already been performed to determine the properties of clumps associated with known star formation sites, such as infrared dark clouds (IRDCs) (Traficante et al. 2015) and MALT90 clumps (Guzmán et al. 2015), as well as generally over the inner Galactic plane (Elia et al. 2017).

As the most appropriate masers to exclusively select high-mass protostars in a constrained range of evolutionary states, we identify a large sample of protostellar clumps in a 
similar stage of evolution through association with a class II methanol maser. The availability and Galactic scale of the catalogues from both Hi-GAL and the MMB survey allows us to select a sample of sufficient size to fully characterise the properties of clumps hosting $6.7 \mathrm{GHz}$ methanol masers. As this is a large sample of objects (several hundred), we adopt analysis techniques to compare populations of objects and identify statistically significant differences in the underlying distributions of properties, rather than compare individual sources. The first portion of this work (Sections 3-5) details the identification and infrared characterisation of this population of objects. We then address the question of whether the presence of a methanol maser in a protostellar clump has any significance, making use of mid-infrared data principal component analysis to identify a sample of objects with identical properties to a methanol maser host clump but lacking a strong 6.7 GHz maser (Sections 6-7).

Section 8 includes additional star formation masers to identify differences in populations of sources within the sample of $6.7 \mathrm{GHz}$ host clumps. Previous work has found correlation between the appearance of and properties of masers during the progression of the star formation process in a clump (e.g. Breen et al. 2010; Titmarsh et al. 2014, 2016). This work does not aim to characterise the Galactic populations of star formation masers themselves, but presents an investigation into evolutionary differences between clumps with a variety of other maser species secondary to the $6.7 \mathrm{GHz}$ line. The relationship between clump and maser properties is also discussed.

\section{DATA SETS}

\subsection{Methanol MultiBeam survey}

The MMB survey completely searched Galactic longitudes from $-174^{\circ}$ to $60^{\circ}$ through the Galactic centre, and latitudes $|b| \leq 2^{\circ}$ for $6.7 \mathrm{GHz}$ methanol masers (Green et al. 2009). A total of $9726.7 \mathrm{GHz}$ methanol masers were detected above the $3-\sigma$ detection limit of $0.51 \mathrm{Jy}$ and are presented in a series of five catalogue papers (Caswell et al. 2010; Green et al. 2010; Caswell et al. 2011; Green et al. 2012; Breen et al. 2015). The catalogue papers provide precise maser positions derived from targeted interferometric observations accurate to 0.4 arcsec, as well as peak flux density information. The integrated flux densities are provided for the full catalogue in Breen et al. (2015).

In this paper, we adopt the distances for MMB sources published in Green \& McClure-Griffiths (2011) and Green et al. (2017). These are primarily kinematic distances, with near-far ambiguities resolved through $\mathrm{HI}$ self absorption, and utilise the kinematic parameters of Reid et al. (2016). As reported in Green et al. (2017), the near-far kinematic distance ambiguity is resolved for 778 of the MMB sources, and we exclude the remaining sources with near-far ambiguities from any distance-dependent analysis.

\subsection{Additional maser studies}

A targeted follow-up towards each MMB source to search for a second class II methanol line at $12.2 \mathrm{GHz}$ has been completed, with the catalogues published in Breen et al. (2012a,b, 2014, 2016). A total of $43812.2 \mathrm{GHz}$ masers were detected (45.3 per cent of sources). The MMB survey also covered the $6035 \mathrm{MHz}$ excited-state maser transition of $\mathrm{OH}$, with 127 ex-OH masers detected over the southern Galactic plane (Avison et al. 2016). The most recent follow-up to the MMB survey is the search for water masers associated with the $6.7 \mathrm{GHz}$ masers by Titmarsh et al. (2014, 2016, hereafter the Titmarsh et al. sample). This survey targeted all 217 MMB masers between Galactic longitudes $-19^{\circ}$ and $20^{\circ}$ with the Australia Telescope Compact Array (ATCA), finding 110 to be associated with a $22 \mathrm{GHz}$ water maser.

Additional water masers have been detected by HOPS (Walsh et al. 2011), which surveyed $100 \mathrm{deg}^{2}$ of Galactic plane between $-70^{\circ}<l<30^{\circ}$ with the Mopra Radio Telescope between 19.5 and $27.5 \mathrm{GHz}$ at lower sensitivity than the Titmarsh et al. studies. As water masers are less localised than methanol masers, precise positions were obtained from follow-up observations with ATCA (Walsh et al. 2014). These were used to group detected masers into sites, 435 of which are associated with star formation. For this work, we use the association of these masers with MMB masers given in Breen et al. (2018).

The final maser survey that we make use of is the SPLASH survey (Dawson et al. 2014) of ground-state hydroxyl masers, with the initial survey with the Parkes telescope between $-28^{\circ}<l<10^{\circ}$ through the Galactic centre and Galactic latitudes $-2^{\circ}$ and $+2^{\circ}$ detecting $\sim 600 \mathrm{OH}$ maser sites. Qiao et al. (2016, 2018) obtained accurate interferometric positions with the ATCA for $\mathrm{OH}$ masers in two regions of $334^{\circ}<l<344^{\circ}$ and $-5^{\circ}<l<5^{\circ}$, and also identify associations between the $\mathrm{OH}$ masers and MMB masers in these ranges. Only the main line transitions of 1665 and $1667 \mathrm{MHz}$ are associated with high-mass star forming regions.

\subsection{Hi-GAL compact source catalogues}

The Hi-GAL survey mapped the entire Galactic plane in a $2^{\circ}$ strip following the Galactic warp in five wavelengths in the far-infrared (Molinari et al. 2010b,a). Observations at $70,160,250,350$ and $500 \mu \mathrm{m}$ were carried out simultaneously, with angular resolutions of 10.0, 13.6, 18.0, 24.0 and 34.5 arcsec in each band respectively (Traficante et al. 2011). Following map creation, photometric catalogues of compact sources extracted using the CuTEx algorithm (Molinari et al. 2011) have been published by Molinari et al. (2016a) for each wavelength for the inner Galactic plane. These catalogues contain 123210, 308 509, 280685, 160972 and 85460 sources at 70, 160250,350 and $500 \mu \mathrm{m}$ respectively between Galactic longitudes $-71^{\circ}$ and $67^{\circ}$ through the Galactic centre. The Hi-GAL compact source catalogues made use of in this work are similarly extracted with $\mathrm{Cu}-$ TEx but cover the entire Galactic plane (S. Molinari et al., priv. comm.). For the full Galactic plane, the numbers of objects in the compact source catalogues for each wavelength increases to 158092 at $70 \mu \mathrm{m}, 580295$ at $160 \mu \mathrm{m}, 468394$ at $250 \mu \mathrm{m}, 251679$ at $350 \mu \mathrm{m}$ and 129489 at $500 \mu \mathrm{m}$.

In this work, we carry out a multi-wavelength analysis using the CuTEx catalogues to derive the physical properties of massive clumps hosting methanol masers. Elia et al. (2017) have derived the properties of the population of massive clumps visible with Hi-GAL and we use their results 
to provide a sample of objects against which to compare maser-hosting clumps.

\subsection{Mid-infrared data}

The FIR observations are complemented by data sets in the MIR from the Wide-field Infrared Survey Explorer (WISE) and Spitzer satellites. The GLIMPSE and MIPSGAL surveys carried out with the Spitzer satellite provide coverage of the inner Galactic plane at high resolution (Churchwell et al. 2009; Benjamin et al. 2003; Carey et al. 2009), with the two surveys covering $295^{\circ}<l<65^{\circ}$ and $298^{\circ}<l<63^{\circ}$ respectively for at least $|b|<1^{\circ}$ over the survey range. For this study, we make use of the GLIMPSE I, II and 3D catalgoues at $8 \mu \mathrm{m}$ and MIPSGAL $24 \mu \mathrm{m}$ point source catalogues produced by Gutermuth \& Heyer (2015).

At a lower resolution, the WISE satellite has surveyed the entire Galactic plane, with the most recent data set AllWISE combining both the initial WISE and NEOWISE data sets (Wright et al. 2010; Mainzer et al. 2011). To supplement the inner Galactic plane coverage of the MIPSGAL catalogue, the AllWISE point source catalogue is also used to identify counterparts at $22 \mu \mathrm{m}$.

In addition to the point-like counterparts identified in the mid-infrared, we also make use of the catalogue of Extended Green Objects (EGOs) of Cyganowski et al. (2008). These are defined as regions of excess extended emission in the $4.5 \mu \mathrm{m}$ band of the original Spitzer GLIMPSE survey. As only the GLIMPSE I data was used for the (Cyganowski et al. 2008) catalogue, the Galactic plane coverage is limited to the regions of $295^{\circ}<l<350^{\circ}$ and $10^{\circ}<l<65^{\circ}$ for $|b|<1^{\circ}$. Cyganowski et al. (2009) and Cyganowski et al. (2011) find class I methanol masers, commonly associated with outflow activity, to spatially coincide with EGO emission towards high-mass star forming regions. Predominantly tracing emission from species shocked through outflow interactions, EGOs provide another possible signpost of the evolutionary state of a $6.7 \mathrm{GHz}$ host clump alongside the secondary masers (Reach et al. 2006; De Buizer \& Vacca 2010).

\section{IDENTIFICATION OF COUNTERPARTS}

\subsection{Hi-GAL compact sources}

The thermal dust emission associated with the clump hosting each maser may be identified in the catalogues of compact sources visible in each of the wavelengths in the HiGAL survey. Association with a methanol maser is determined through on-sky position. Due to the degradation of resolution with increasing wavelength present in the Hi-GAL data, the maximum allowed distance between the centroid of a clump and the maser position is varied with wavelength when identifying maser counterparts.

This reduces the number of false associations at short wavelengths and avoids missed counterparts at long wavelengths due to the positional uncertainty. For each of the Hi-GAL catalogues, a counterpart is assigned to a maser if the source centroid is separated by less than half of the corresponding Hi-GAL beam widths from the maser position. The maximum angular separations are 5.1, 6.8, 9.0, 12.0 and
Table 1. Infrared counterparts to the $972 \mathrm{MMB}$ masers found in each of the single wavelength Hi-GAL source extractions. The number of sources with a counterpart identified within the maximum counterpart angular distance $\theta$ in Column 2 is reported in Column 3 for each band. The number is also given as a percentage of the maser sample in Column 4 . The bottom row gives the number of MMB sources with a counterpart identified in $\geq 1$ Hi-GAL band.

\begin{tabular}{lccc}
\hline$\lambda[\mu \mathrm{m}]$ & $\theta\left[{ }^{\prime \prime}\right]$ & No. with counterpart & $\%$ \\
\hline 70 & 5.1 & 859 & 88.4 \\
160 & 6.8 & 873 & 89.8 \\
250 & 9.0 & 823 & 84.7 \\
350 & 12.0 & 826 & 85.0 \\
500 & 17.3 & 822 & 84.6 \\
Any & - & 930 & 95.7 \\
\hline
\end{tabular}

17.25 arcsec at $70,160,250,350$ and $500 \mu \mathrm{m}$ respectively. In the instance that several counterparts are identified, the source with the smallest angular separation from the maser coordinates is selected. Table 1 shows the number of masers assigned a counterpart in each wavelength, with $>84 \%$ of masers detected with a counterpart in any single band. Overall, nearly all sources $(95.7 \%)$ are associated with a Hi-GAL compact source in at least one band.

For the longest wavelength Hi-GAL catalogues, a greater number of false associations between masers and sources in this catalogue will be returned due to the increased beam size. The overall percentage associated at $500 \mu \mathrm{m}$ is however less than at other wavelengths, most likely due to the comparatively poor resolution resulting in missed detections. For clumps hosting evolved protostellar sources, such as those capable of sustaining a class II methanol maser, the $160 \mu \mathrm{m}$ emission is expected to be close to the peak of the thermal SED. In line with this, we find the number of sources with a counterpart recovered to be greatest for $160 \mu \mathrm{m}$.

\subsection{Multi-wavelength sample selection}

The sample of masers studied further in this work is selected based on the visibility of compact emission over several Hi-GAL wavelengths. Since Class II methanol masers are pumped by radiation at $\sim 70 \mu \mathrm{m}$ (Sobolev et al. 2005), their host clumps are expected to be 'protostellar' in nature, visible as both compact emission from the cool dust envelope at wavelengths $\geq 160 \mu \mathrm{m}$ and at $70 \mu \mathrm{m}$ from a warmer inner component (Motte et al. 2010). In order to fit to the spectral energy distribution (SED) of the dust envelope of a protostellar clump, a detection in at least 3 wavelengths $\geq 160 \mu \mathrm{m}$ is required, as the emission at $70 \mu \mathrm{m}$ is neither optically thin nor tracing the same cold material.

Although a high percentage of sources are associated with a $500 \mu \mathrm{m}$ counterpart, this is artificially increased due to the larger beam size including false associations with sources that may not truly host the methanol maser. In these cases, the $500 \mu \mathrm{m}$ counterparts may belong to cold dust condensations that do not show any evidence of emission at $\leq 160 \mu \mathrm{m}$ associated with an embedded protostar. Therefore, requiring a catalogued $500 \mu \mathrm{m}$ detection severely limits our multi-wavelength sample size as all associations that are less likely to be true associations are removed 
through shorter wavelength requirements, and the percentage of maser sources with a $500 \mu \mathrm{m}$ counterpart falls below that of the other bands. So, the sample analysed further are defined as masers associated with a counterpart in 70,160 , 250 and $350 \mu \mathrm{m}$ only.

Subject to these constraints, $7316.7 \mathrm{GHz}$ methanol masers (72.5 per cent) are identified with infrared emission in all bands between 70 and $350 \mu \mathrm{m}$ In addition to these sources, we also note that 22 masers (2.2 per cent) are identified with emission at wavelengths between 160, 250 and $350 \mu \mathrm{m}$ but lack a detected $70 \mu \mathrm{m}$ counterpart. Elia et al. (2017) show that a deeper targeted extraction at $70 \mu \mathrm{m}$ towards such sources is likely to reveal a counterpart or provide an upper limit on the flux from any warm component present. By visual inspection of the Hi-GAL maps, we confirm that these maser sources do all have a counterpart at $70 \mu \mathrm{m}$ but are not recovered by CuTEx.

Methanol maser sources detected in the FIR in previous studies may also be missing from the sample in this work due to the saturation of the Hi-GAL maps at 250 and/or $350 \mu \mathrm{m}$. An example of this are the two maser-hosting protostellar clumps in the infrared dark cloud SDC335, with Avison et al. (2015) making use of the 70 and $160 \mu \mathrm{m}$ Hi-GAL data only. In total, 80 sources are removed due to unreliable fluxes reported in the Hi-GAL catalogues.

The sample is further refined to exclude sources displaying irregular FIR SEDs as it will not be possible to fit these with the simple model chosen to describe the objects. As the CuTEx algorithm used to produce the Hi-GAL catalogues performs source deblending, such sources are likely to be false associations giving rise to the unphysical shape rather than blending within a Hi-GAL beam. For prestellar and protostellar cores, the dust envelope of a source is expected to have a SED peaking at $\leq 250 \mu \mathrm{m}$. Defining the [250-350] $\mu \mathrm{m}$ colour of a source as the logarithmic ratio of 250 to $350 \mu \mathrm{m}$ flux, we use this property to assess the validity of the infrared fluxes assigned to maser.

Prior to the calculation of the [250-350] $\mu \mathrm{m}$ colour of a source, the fluxes at long wavelengths require scaling to ensure that the flux from the same volume of material is considered at all wavelengths, as excess gas is included if a source is unresolved. The size at $250 \mu \mathrm{m}$ is taken to most reliably trace the size of the cold clump envelope, the fluxes at $350 \mu \mathrm{m}$ are scaled according to the relation

$F_{v}^{S E D}=F_{v} \times \frac{\mathrm{FWHM}_{250}^{d e c}}{\mathrm{FWHM}_{v}^{d e c}}$

where the $\mathrm{FWHM}^{\text {dec }}$ values are the deconvolved circularised FWHM source sizes (Nguyên Luong et al. 2011) given in the Hi-GAL catalogues. The sources are assumed to be resolved at 70 and $160 \mu \mathrm{m}$ and no scaling is applied. On average, the scaled flux at $350 \mu \mathrm{m}$ is 73 per cent of the original counterpart flux, with a standard deviation of 13 per cent. The overall effect is to steepen the SED of each source between $250 \mu \mathrm{m}$ and $350 \mu \mathrm{m}$, i.e. shifting the [250-350] $\mu \mathrm{m}$ colour towards more positive values, with an example shown in Figure 1.

We exclude sources of negative [250-350] $\mu \mathrm{m}$ colour (more emission at $350 \mu \mathrm{m}$ than $250 \mu \mathrm{m}$ ), as these are irregular SEDs given the expected peak emission wavelength. Imposing further constraints based on the source colours is not possible, as the temperature dependence of the peak position may lead to either increasing or decreasing SEDs in all other consecutive wavelength bands. Initially 24 of the 731 sources fall below the colour limit at 0 , reducing to 4 following flux scaling. In conjunction with the removal of sources with unreliable fluxes, a total of 84 sources are removed from the sample, giving a sample size of 647 for further analysis.

\subsection{Mid-infrared counterparts}

To extend the spectral energy distribution, positional association was similarly used to search the mid-infrared point source catalogues described in Section 2.4 for all 647 maser sources appearing in the four required Hi-GAL bands with reliable fluxes. The $24 \mu \mathrm{m}$ MIPSGAL catalogue was initially searched for counterparts to the sample of masers identified above using a half-beam association radius of $3.0 \mathrm{arcsec}$ (Gutermuth \& Heyer 2015). Of the 627 sources falling within the MIPSGAL survey range, only 40.8 per cent of the sample (304 sources) were found to have an associated MIPSGAL $24 \mu \mathrm{m}$ point source. As for the $70 \mu \mathrm{m}$ catalogues, Elia et al. (2017) also performed a deeper targeted extraction in the MIPSGAL data towards their protostellar objects to recover faint sources to either identify missing counterparts, or provide an upper limit on the $24 \mu \mathrm{m}$ flux.

However, the masers lacking $24 \mu \mathrm{m}$ counterparts show a trend towards higher FIR luminosities (see Section 5.3). As the FIR luminosity derived from the Hi-GAL data correlates with the luminosity at $24 \mu \mathrm{m}$, this implies that saturation of the MIPSGAL images is a plausible cause for the small fraction of sources associated with a counterpart, rather than the failing to detect faint $24 \mu \mathrm{m}$ sources (Dunham et al. 2008). Visual inspection of the MIPSGAL images confirms that this is the case towards a majority of the sources.

The AllWISE point source catalogue at $22 \mu \mathrm{m}$ was also searched for counterparts as a substitute for those lacking $24 \mu \mathrm{m}$ sources. This data is at much lower resolution, with a beam size of 24.0 arcsec, but does not suffer from the same saturation effects. Further to this, AllWISE covers the full range of coordinates of the sample. Although the lower resolution AllWISE data will not detect the small and faint counterparts detectable in the MIPSGAL data, the detection of bright sources and extended survey range recovers additional counterparts. A total of 451 counterpart sources are found in the $22 \mu \mathrm{m}$ AllWISE catalogue, 280 of which were not assigned a MIPSGAL counterpart previously.

For the purpose of determining the existence of a $\sim 20 \mu \mathrm{m}$ counterpart and estimating the flux by tabulated integration, the $22 \mu \mathrm{m}$ WISE and $24 \mu \mathrm{m}$ MIPSGAL data points are used interchangeably. Combining the WISE and MIPSGAL detections finds a majority (518, 80.1 per cent) of maser host sources to be associated with a counterpart at $\sim 20 \mu \mathrm{m}$. For the masers with a counterpart identified in both the MIPSGAL and AllWISE catalogues, the MIPSGAL counterpart is used preferentially. For sources with both a MIPSGAL and AllWISE counterpart found, a comparison of the two flux values finds no systematic differences.

The Spitzer GLIMPSE I, II and 3D point source catalogues were similarly used to assign 304 counterparts at $8 \mu \mathrm{m}$ within 2.0 arcsec of each maser coordinate. The association of $6.7 \mathrm{GHz}$ methanol masers with $8 \mu \mathrm{m}$ counterparts has previously been investigated by Gallaway et al. (2013). 
However, these associations are only performed for the subset of MMB masers with interferometric positions available at the time of publication. We do not use these associations but match to the GLIMPSE catalogues to cover the full MMB range in addition to the Hi-GAL protostellar objects, and ensure that the matching criteria are consistent for the two samples. The numbers of sources with an MIR counterpart in each of these surveys is shown in Table 2, alongside the number of the methanol maser hosts within each survey region.

Approximately half (52.1 per cent) of the maser sources bright at $\sim 20 \mu \mathrm{m}$ also have an $8 \mu \mathrm{m}$ GLIMPSE object assigned to them and therefore the presence of an $24 \mu \mathrm{m}$ source does not necessarily indicate that emission is expected at $8 \mu \mathrm{m}$. Conversely, most sources ( 88.8 percent) visible at $8 \mu \mathrm{m}$ also have a MIPSGAL or AllWISE counterpart.

Gallaway et al. (2013) previously reported that 83 per cent of methanol masers are associated with emission in at least one of the 4 GLIMPSE bands (67 per cent in all bands). When performing the same matching against the GLIMPSE point source catalogues, the authors recovered a counterpart in any GLIMPSE band for only 55 per cent of the MMB masers. This difference is attributed to the lack of extended or slightly extended emission included in the point source catalogues, as is common towards maser sources, and report that the number of MIR counterparts to the MMB masers increases by a factor of approximately 2 if such sources are included alongside point-like counterparts. Performing targeted source extraction and inspection towards both the maser and protostellar samples to recover extended emission is beyond the scope of this paper. Our result that 48.3 per cent of methanol masers are associated with an $8 \mu \mathrm{m}$ counterpart is therefore an underestimate of the true number of sources bright at $8 \mu \mathrm{m}$.

\section{INFRARED CLUMPS ANALYSIS}

\subsection{SED fitting}

Within the far infrared regime, each methanol maser host clump is taken to emit thermally at the dust temperature as a single temperature modified blackbody, or greybody. This approximation describes the emission well for wavelengths $\geq 160 \mu \mathrm{m}$, but the $70 \mu \mathrm{m}$ flux is found to trace the warm embedded protostellar component and not the emission from the envelope (Motte et al. 2010; Dunham et al. 2008). Additionally, the emission at $70 \mu \mathrm{m}$ is not necessarily optically thin and cannot be reliably fitted without also modelling optical depth effects.

At wavelengths $\geq 160 \mu \mathrm{m}$, the emission is optically thin and the observed intensity of emission from a clump is given by

$S_{v}=\frac{M \kappa_{v}}{d^{2}} B_{v}(T)$.

where $B_{v}(T)$ is the Planck function evaluated at the observation frequency $v$ for clump temperature $T, M$ is the total gas+dust mass of the envelope and $d$ is the distance to the clump. The dust mass opacity coefficient $\kappa_{v}$ given in units of $\mathrm{cm}^{2} \mathrm{~g}^{-1}$ is frequency dependent and assumed to follow the

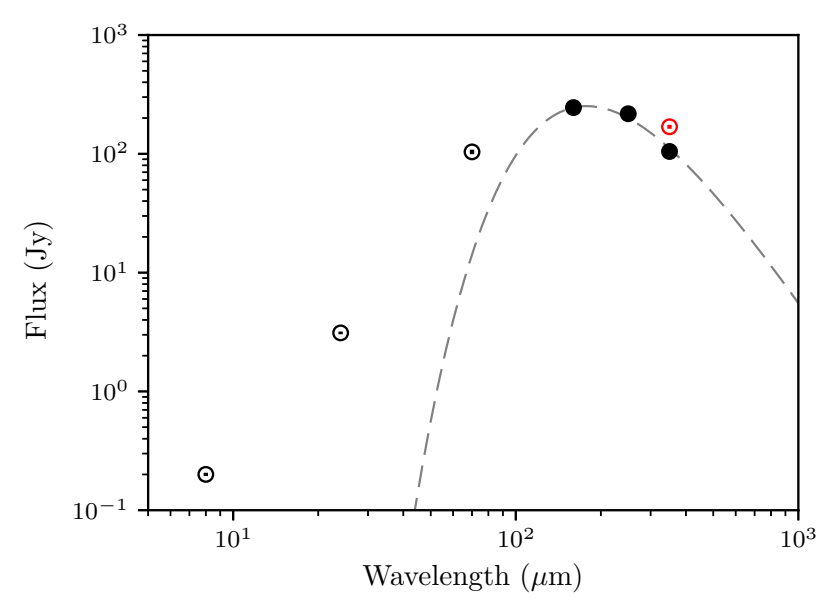

Figure 1. The fluxes integrated over the source size in the HiGAL maps, for a typical maser hosting clump in the sample. The filled circles are the data points used for fitting to the SED at 160, 250 and $350 \mu \mathrm{m}$, with the unscaled flux at $350 \mu \mathrm{m}$ shown in red. The data points likely originating from a warmer inner component at 8,24 and $70 \mu \mathrm{m}$ are marked as open circles and are not used for fitting. The grey dashed line is the fitted greybody for this source, returning a temperature of $17.0 \mathrm{~K}$ and a clump envelope mass of $806 \mathrm{M}_{\odot}$, given that the distance to the host maser is $2.7 \mathrm{kpc}$.

scaling relation

$\kappa=\kappa_{0}\left(\frac{v}{v_{0}}\right)^{\beta}$

where the values of $\kappa_{0}$ and $v_{0}$ are set to $0.005 \mathrm{~cm}^{2} \mathrm{~g}^{-1}$ and $230 \mathrm{GHz}$ respectively for a gas-to-dust ratio of 100 (Preibisch et al. 1993). The spectral index $\beta$ is fixed rather than fitted as the limited number of data points for each source does not allow reliable determination and the degeneracy between $\beta$ and $T$ cannot be accounted for. A value of 1.8 is chosen as this was found to best characterise the shape of the observed SED of the clumps, consistent with previous work (e.g. Juvela et al. 2015, 2018; Guzmán et al. 2015). A greybody is fitted to each constructed SED by least-squares minimisation using the Levenberg-Marquardt algorithm to return best fit values of $T$ and $\frac{M}{d^{2}}$. For masers with a known distance from the data described in Section 2.1, values of physical parameters such as total mass and radius are also obtained for a clump. An example of a the FIR SED of a typical maser host source and the fitted greybody is shown in Figure 1.

\subsection{Calculating luminosity}

For this analysis, the FIR luminosity of a source is defined as the luminosity in the range $70-500 \mu \mathrm{m}$. We do not include the mid-infrared fluxes in this luminosity calculation to ensure that the integral is evaluated over the same interval for all sources in the sample. Although distance-dependent quantities themselves, parameters such as the ratio of $L$ to $M$ may still be evaluated for sources lacking a distance value.

Taking the total FIR luminosity as the area under the fitted greybody SED returns an estimate of the luminosity originating from the dust envelope of the clump. This does 
Table 2. The single wavelength searches for maser counterparts in GLIMPSE $8 \mu \mathrm{m}$, MIPSGAL $24 \mu \mathrm{m}$ and AllWISE $22 \mu \mathrm{m}$ point source catalogues. The intersection of each survey coverage with the MMB survey is given in the second column. The number of FIR detected masers falling within the range of each survey is stated, alongside the number with a counterpart assigned. Counterparts were assigned using a maximum distance of one half-beam size for each survey. The last two rows indicate the total number of the maser sources with a visible counterpart in either of the $\sim 20 \mu \mathrm{m}$ catalogues and the subset of these also associated with $8 \mu \mathrm{m}$ emission.

\begin{tabular}{|c|c|c|c|}
\hline Survey (wavelength $[\mu \mathrm{m}]$ ) & Common coverage & Masers in range & No. with counterpart (\%) \\
\hline GLIMPSE I, II, 3D (8) & $295^{\circ}<l<60^{\circ},|b| \leq 1$ & 629 & $304(48.3)$ \\
\hline MIPSGAL (24) & $298^{\circ}<l<60^{\circ},|b| \leq 1$ & 627 & $238(40.0)$ \\
\hline AllWISE (22) & $186^{\circ}<l<60^{\circ},|b| \leq 2$ & 647 & $451(69.7)$ \\
\hline AllWISE (22) or MIPSGAL (24) & $186^{\circ}<l<60^{\circ},|b| \leq 2$ & 647 & $518(80.1)$ \\
\hline AllWISE (22)/MIPSGAL (24) \& GLIMPSE (8) & $295^{\circ}<l<60^{\circ},|b| \leq 1$ & 629 & $270(42.9)$ \\
\hline
\end{tabular}

not reliably describe the total far-infrared luminosity of a source as an excess of energy is likely to be emitted by a warmer inner component due to the embedded protostar at shorter wavelengths, including $70 \mu \mathrm{m}$ (Dunham et al. 2008). Despite this, 26 sources show a deficiency in $70 \mu \mathrm{m}$ flux relative to the greybody estimate, which can be attributed to unreliable recovery of the true flux at $70 \mu \mathrm{m}$ due to complex environments and source blending (e.g. Elia et al. 2013; Molinari et al. 2016a; Persi et al. 2016). We therefore define the FIR luminosity of a source $L_{\mathrm{FIR}}$ as the integral under the tabulated data points in this range calculated, supplementing the four required Hi-GAL fluxes with a $500 \mu \mathrm{m}$ flux returned by extrapolating the best-fit greybody, as the envelope described by the single temperature greybody is responsible for emission at this wavelength. We perform tabulated integration using a 5-point Newton-Cotes formula to allow for deviation from ideal greybody behaviour at $70 \mu \mathrm{m}$ and better characterise the total energy emitted in the FIR by the clump (see Section 5.3).

Throughout the following sections the properties of this sample of maser host clumps are shown alongside the same distributions for the general Galactic protostellar clump population visible with Hi-GAL, defined by Elia et al. (2017). Prior to comparison with the protostellar sample, it should be noted that there is a small difference in how the luminosity of the two samples (masers and protostars) is calculated. For this work, the maser clump luminosity is strictly defined as the integral given by the tabulated HiGAL data between 70 and $500 \mu \mathrm{m}$, and so is a measure of the far-infrared luminosity. On the other hand, Elia et al. (2017) calculate the bolometric luminosity of each source by including the flux at wavelengths outside the Hi-GAL wavelength range. For the sources in the protostellar sample with a counterpart detected in either the MIPSGAL, $22 \mu \mathrm{m}$ WISE or $21 \mu \mathrm{m} M S X$ (Egan et al. 2003) data, the range of fluxes used to calculate source luminosity have been extended to have a lower limit of $\sim 20 \mu \mathrm{m}$. For all sources, Elia et al. (2017) calculate the luminosity at wavelengths $\geq 160 \mu \mathrm{m}$ from the greybody fit and use the tabulated fluxes to calculate the luminosity contribution at shorter wavelengths. For the maser-bearing sources associated with $24 \mu \mathrm{m}$ emission, we find an average increase in luminosity by a factor of $2.0 \pm 0.78$ compared to the far-infrared luminosity used here. As Elia et al. (2017) find only $10 \%$ of sources to be MIR dark (i.e. lacking a counterpart at $\sim 20 \mu \mathrm{m}$ ), this correction factor is likely to apply to most sources. Given that the infrared emission is responsible for the pumping of the class II methanol maser line, this luminosity is the most appropri-

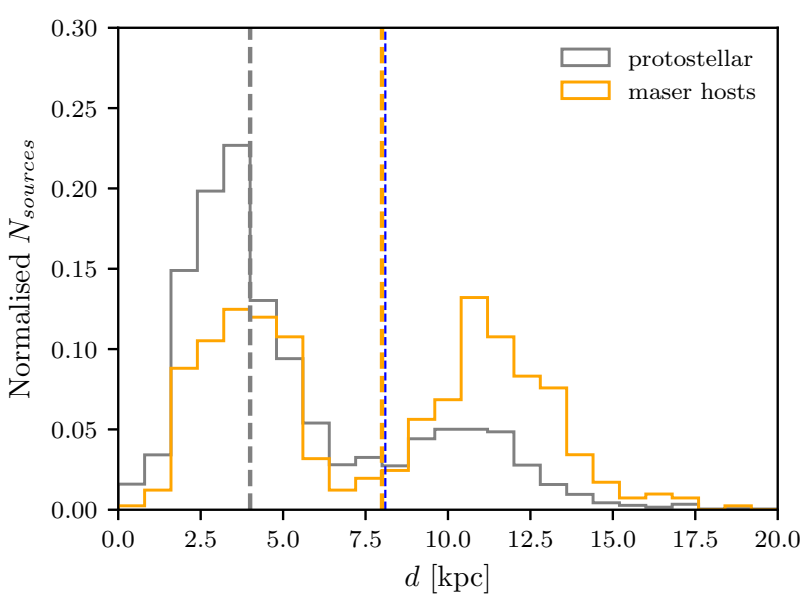

Figure 2. Heliocentric distance distributions of the protostellar (grey) and maser (orange) samples, with the median distances of $4.0 \mathrm{kpc}$ and $8.0 \mathrm{kpc}$ marked in respective colours. The assumed distance to Galactic centre at $8 \mathrm{kpc}$ is marked in blue.

ate for this work, although typically evolutionary diagnostics such as $L / M$ are designed for use with the bolometric luminosity.

\section{FIR RESULTS}

The derivation of clump properties for the $6.7 \mathrm{GHz}$ hosts from their FIR fluxes allows us to compare this sample against existing catalogues of high-mass star forming clumps. Within the 22,426 protostellar sources reported by Elia et al. (2017), we select the 22,293 sources satisfying the same multi-wavelength (70 to $350 \mu \mathrm{m})$ visibility criteria as the maser sample, and restrict to the Galactic longitude and latitude range of the MMB survey. Additionally, we only consider the 25 per cent of the above sources with 'good' distances (i.e. resolved between near and far through the use of other tracers) as the assumption of far kinematic distance applied to the remaining sources may artificially increase in the number of sources at high luminosities and masses. The distance distributions of the two samples are shown in Figure 2. Each displays bimodality around the Galactic centre, which is unsurprising as IR observations and kinematic distance resolution is challenging in this direction, resulting in a dip at the assumed distance to Galactic cen- 


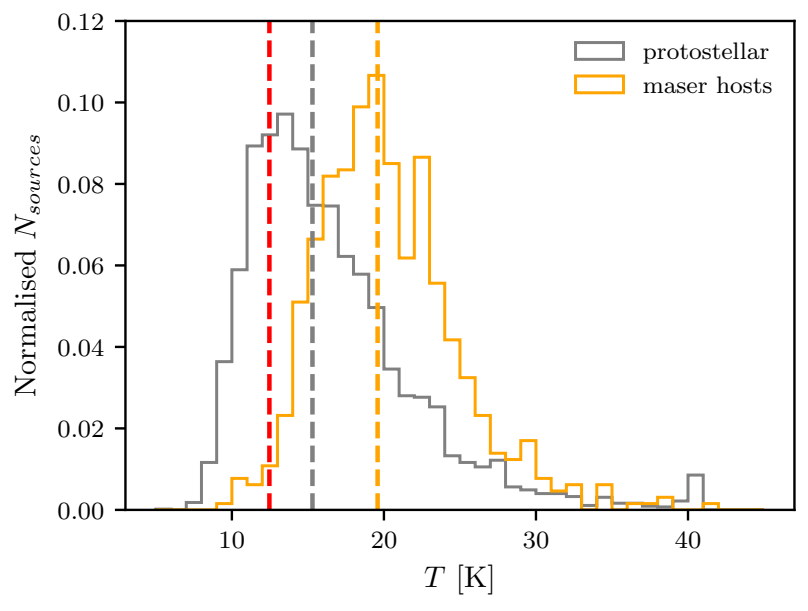

Figure 3. Distribution of temperatures determined by greybody fitting to the SED of clumps for the protostellar (grey) and maser (orange) samples. The sample medians of $19.5 \mathrm{~K}$ and $15.3 \mathrm{~K}$ are also marked for the maser and protostellar sample respectively. The threshold clump temperature taken to be required to sustain a class II methanol maser is marked in red at $12.5 \mathrm{~K}$.

tre of $8.0 \mathrm{kpc}$. The symmetry in the distances of the MMB sources between the near and far side of the Galaxy supports that the MMB survey has recovered the majority of Galactic methanol masers. However, a significant shift towards distances on the near side of the Galactic plane is seen for the protostellar sample relative to the maser hosts. This reflects the ability of Hi-GAL to detect sources of a given angular size, corresponding to a larger physical radius at further distances, and the primary methods used to confirm distances or resolve kinematic ambiguities, such as association with absorption features (Elia et al. 2017). The effects of the bimodality in distance are discussed in Section 5.4.

We select this sample of 5497 protostellar objects for comparison, hereafter the 'protostellar sample', with the aim to determine whether the subset of protostellar sources hosting a visible methanol maser occupies a constrained space within the total population of protostellar objects in the inner Galactic plane.

The following sections detail the results for the individual properties calculated for the maser host clumps, compared against the protostellar sample in each case. Table 4 presents the distribution medians and standard deviations for all properties. Approximate thresholds on the clump properties required to host a methanol maser are given in each section, and also included in this table. In each case, these represent requirements of a protostellar environment on the clump scale, rather than the conditions in the circumstellar region of an individual massive protostar, to sustain maser activity.

\subsection{Temperature and mass surface density}

For clumps in the sample, the distance independent quantities of temperature and mass surface density $\Sigma$ are obtained for all sources following SED fitting. The methods adopted by Elia et al. (2017) to characterise their protostellar sources are similar to our own, both using the $250 \mu \mathrm{m}$ angular clump size to define physical size and modelling each SED as a modified blackbody. A key difference in our methods is the choice of spectral index $\beta$ and reference dust mass opacity $\kappa$.

To correct for differences in $\beta$ when considering FIR SEDs fitted with a modified blackbody covering the peak of emission, Guzmán et al. (2015) give a relationship between spectral index and temperature of

$\frac{h v_{\text {peak }}}{k_{\mathrm{B}} T_{\mathrm{d}}} \simeq \beta+3$

where $T_{\mathrm{d}}$ is the dust temperature, $v_{\text {peak }}$ is the wavelength at which the SED peaks, and $k_{\mathrm{B}}$ and $h$ are the Boltzmann and Planck constants respectively, with this relationship accurate to 10 per cent for values of $\beta>1$. Following the method used by Guzmán et al. (2015) to compare temperatures derived from the far-infrared with different values of $\beta$, we obtain a scaling of $T_{\beta=2.0}=0.96 T_{\beta=1.8}$. The median temperature of the maser sample decreases to $18.7 \mathrm{~K}$ under this correction, and does not significantly affect the conclusion that the maser sources are significantly warmer. Assuming a worst-case of 10 per cent uncertainty on this scaling, the largest possible decrease in median temperature from the use of a different $\beta$ is $2.7 \mathrm{~K}$ which, although not completely negligible, is also significantly less than the $4.2 \mathrm{~K}$ offset in temperature seen between the protostellar and maser clumps. We therefore do not consider the choice of $\beta$ to significantly impact the results obtained throughout this work.

When comparing median $\Sigma$, masses or thresholds between different works, it is important to be aware of the offset factor which the adoption of different values and forms for the dust mass opacity can introduce. For the dust mass opacity, Elia et al. (2017) use a reference value of $0.1 \mathrm{~cm}^{2} \mathrm{~g}^{-1}$ at $300 \mu \mathrm{m}$. Rescaling our reference value to $300 \mu \mathrm{m}$ with Equation 3 gives a value of $0.07 \mathrm{~cm}^{2} \mathrm{~g}^{-1}$ and we find that the $\Sigma$ (and mass) presented for a clump in this work offset to higher $\Sigma$ and mass by a factor of 1.4 relative to the protostellar sample due to this difference.

Since all the objects with irregular SEDs and unreliable fluxes in the Hi-GAL compact source catalogues have already been excluded from the sample, the remaining maser sources are well fitted with temperatures up to a maximum of $\sim 42 \mathrm{~K}$. The distribution of fitted $T$ values is shown in Figure 3 which has a median of $19.5 \mathrm{~K}$ for the maser hosts. The protostellar sample has a lower median temperature of $15.3 \mathrm{~K}$. To set thresholds on the properties required for a source to host a methanol maser, we adopt 2-standard deviation lower limits on the distributions of properties of the maser sources, unless otherwise stated. As the presence of a high temperature tail in the temperature distribution causes significant asymmetry, we only use points below the median to calculate the standard deviation and obtain a $2 \sigma$ lower threshold. This gives a minimum temperature for a clump to host a methanol maser of $12.5 \mathrm{~K}$, and is marked in red in Figure 3.

Also making use of the Hi-GAL data, Guzmán et al. (2015) find an average temperature of $18.6 \mathrm{~K}$ for protostellar objects, and the study of Breen et al. (2018) also makes use of these temperature maps to derive a temperature of $\sim 24 \mathrm{~K}$ towards $6.7 \mathrm{GHz}$ methanol masers. Both of these are significantly warmer than the respective temperatures of $15.3 \mathrm{~K}$ and $19.5 \mathrm{~K}$ for the protostellar and maser objects considered 


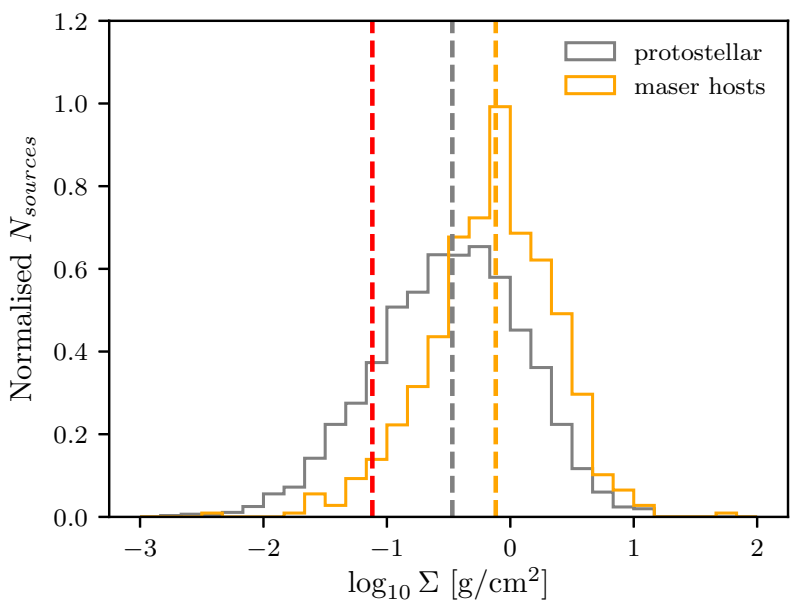

Figure 4. Distribution of mass surface densities calculated from the prefactor returned following greybody fitting and the clump size at $250 \mu \mathrm{m}$. The protostellar sample (grey) has a median of $0.34 \mathrm{~g} \mathrm{~cm}^{-2}$ and the maser sample (orange) has a median of $0.76 \mathrm{~g} \mathrm{~cm}^{-2}$, with a lower limit on the mass surface density required to host a methanol maser (red) set at $0.08 \mathrm{~g} \mathrm{~cm}^{-2}$.

in this work. This offset arises from the difference in aperture that is integrated over to obtain the flux of a source in each Hi-GAL wavelength. Guzmán et al. (2015) assume a fixed aperture at all wavelengths, similar to the clump size at $250 \mu \mathrm{m}$, whereas this work and Elia et al. (2017) allow this size to vary according to the appearance in each map. The size reported in the Hi-GAL compact source catalogues is often significantly smaller at $160 \mu \mathrm{m}$ relative to the size at $250 \mu \mathrm{m}$ for a source. This is therefore a difference in definition rather than a true inconsistency between our two studies. As this work is primarily focused on identifying differences between objects with methanol masers and the protostellar population of Elia et al. (2017), both of which have been characterised using the same flux extraction method, this does not affect the conclusions we draw from this analysis.

Figure 4 shows the distributions of mass surface density $\Sigma$ in logarithmic bins, where $\Sigma=\frac{M}{d^{2}} \frac{1}{\Omega}$ for solid angle $\Omega$ subtended by the clump (calculated from the source FWHM angular size at $250 \mu \mathrm{m}), M$ is the clump mass and $d$ the distance. The median $\Sigma$ for the maser host sample is $0.76 \mathrm{~g} \mathrm{~cm}^{-2}$ with 248 (38 per cent) sources above the approximate threshold of $\Sigma \geq 1 \mathrm{~g} \mathrm{~cm}^{-2}$ for massive star formation proposed by Krumholz \& McKee (2008). Only 21.0 per cent of the protostellar population fall above the same threshold, with a sample median of $0.34 \mathrm{~g} \mathrm{~cm}^{-2}$. Including a correction factor of 1.4 to account for the systematic offset due to the choice of dust mass opacity reduces the maser median value to $0.54 \mathrm{~g} \mathrm{~cm}^{-2}$, and this cannot account for the difference between the two samples.

This threshold is assumed for non-magnetised regions whereas other studies support a lower threshold (e.g. Butler \& Tan 2012; Urquhart et al. 2015; Traficante et al. 2015, 2018), with Tan et al. (2014) suggesting a threshold of $\Sigma \sim 0.1 \mathrm{~g} \mathrm{~cm}^{-2}$ below which massive stars cannot form in the presence of magnetic fields. For the maser and protostellar

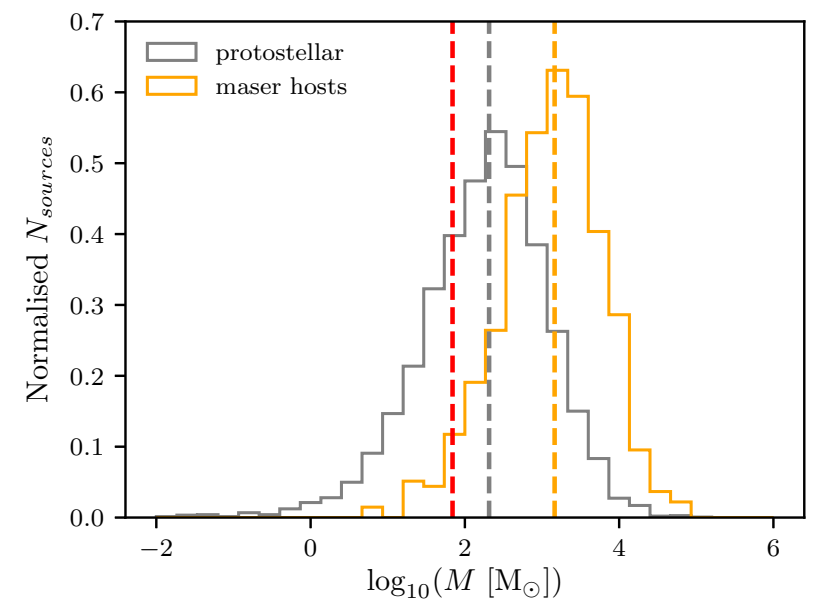

Figure 5. Mass distributions for the protostellar (grey) and maser hosting (orange) samples, with medians of $206 \mathrm{M}_{\odot}$ and $1457 \mathrm{M}_{\odot}$ respectively and a minimum threshold (red) to host a methanol maser set at $69 \mathrm{M}_{\odot}$.

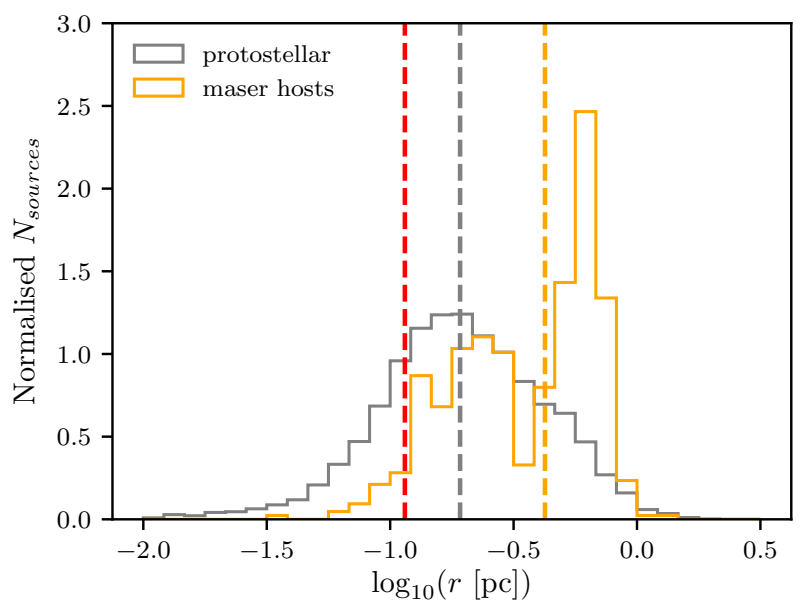

Figure 6. Distributions of physical radii derived from the source size at $250 \mu \mathrm{m}$ for the maser host clumps (orange) and the Galactic protostellar sample (grey), with medians of $0.42 \mathrm{pc}$ and $0.19 \mathrm{pc}$ respectively. The bimodality in radius of the maser sources is due to a bimodality in the distance distribution. The minimum size required for a methanol maser to be present is taken to be $0.11 \mathrm{pc}$ (red).

objects, the percentages of sources above this threshold are 94 and 80 per cent respectively. The maser hosting sources are therefore found to be at a higher median mass surface density than the protostellar sample and a lower limit of $0.08 \mathrm{~g} \mathrm{~cm}^{-2}$ is taken as the requirement for a clump to host a methanol maser.

\subsection{Mass and radius}

For the 511 sources with a reliable distance value and a luminosity $>100 \mathrm{~L}_{\odot}($ see Section 5.3), the mass of a clump may be calculated from the $M / d^{2}$ prefactor previously used 


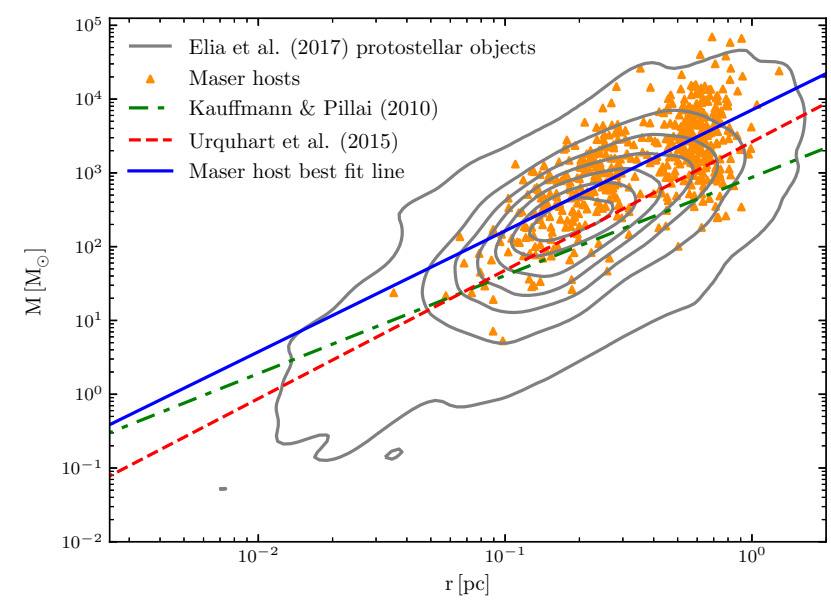

Figure 7. Mass-radius diagram of the maser sources (orange) with the protostellar sources shown as grey contours, with the levels at intervals of $15 \%$ between $1 \%$ and $90 \%$ of the peak density in $M-r$ space. The approximate threshold for star formation to occur in a clump of Kauffmann \& Pillai (2010) is shown as the dash-dotted green line, given by $M(r) \leq 870 \mathrm{M}_{\odot}(r / \mathrm{pc})^{1.33}$. The red dashed line is the power law fit of observed properties towards 6.7 GHz methanol maser clumps by Urquhart et al. (2015), given by $\log \left(\frac{M}{1 \mathrm{M}_{\odot}}\right)=1.74\left(\frac{r}{1 \mathrm{pc}}\right)+3.42$. For comparison, the best fit line to our data is shown as a blue solid line of $\log \left(\frac{M}{1 \mathrm{M}_{\odot}}\right)=(1.64 \pm$ $0.07)\left(\frac{r}{1 \mathrm{pc}}\right)+(3.85 \pm 0.04)$

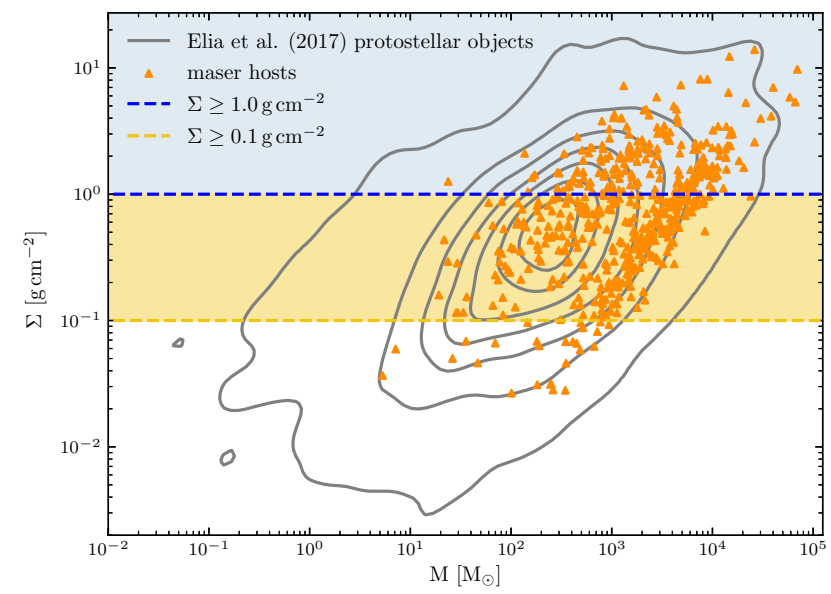

Figure 8. $\Sigma$-M diagram for the protostellar (grey contours, levels at $1 \%, 15 \%, 30 \%, 45 \%, 60 \%, 75 \%$ and $90 \%$ of peak density in $\Sigma$ $\mathrm{M}$ space) and maser hosting (orange) sources. The approximate surface density criterion for star formation to proceed of $\Sigma \geq$ $1.0 \mathrm{~g} \mathrm{~cm}^{-2}$ is marked in blue.

to calculate mass surface density, as well as conversion of the angular source size at $250 \mu \mathrm{m}$ to a physical clump radius. The distributions of the mass and radius are plotted in Figures 5 and 6 respectively. The median mass of the maser hosting clumps is $1457 \mathrm{M}_{\odot}$ with a median radius of $0.42 \mathrm{pc}$, whilst the protostellar sample is less massive and smaller on average, with median values of $206 \mathrm{M}_{\odot}$ and 0.19 pc. Again, we note that the difference in choice of dust mass opacity may bias our results towards higher masses by a factor of 1.4 , although this is a small correction relative to the difference in median mass. The lower limit on mass and radius to host a methanol maser are taken to be $69 \mathrm{M}_{\odot}$ and $0.11 \mathrm{pc}$. We note here that the bimodality in radius seen in Figure 6 , and later separation into two clusters in Figures 7 and 8 , is caused by the underlying bimodal distance distribution shown in Figure 2 and the limited range of angular sizes (1-3 times the PSF in each band, Molinari et al. 2016a) for objects to be included in the Hi-GAL compact source catalogues.

Figure 7 shows the mass of the clumps as a function of the radius, with the Kauffmann \& Pillai (2010) scaling relation shown in green for comparison, with only 6 per cent of the maser hosts falling below this limit. The best fit line of $\log \left(\frac{M}{1 \mathrm{M}_{\odot}}\right)=1.74\left(\frac{r}{1 \mathrm{pc}}\right)+3.42$ obtained by Urquhart et al. (2015) for objects hosting methanol masers is shown in red. For comparison, the line of best fit through our maser hosts is shown in blue and is given by $\log \left(\frac{M}{1 \mathrm{M}_{\odot}}\right)=$ $(1.64 \pm 0.07)\left(\frac{r}{1 \mathrm{pc}}\right)+(3.85 \pm 0.04)$. The differences between the results of Urquhart et al. (2015) and this work are discussed further in Section 5.7.

Mass and radius are both derived using the distance to a source, and this may introduce artificial correlation in $M-R$ space. As mass is proportional to $d^{2}$ and radius to $d$, we would expect to find a relationship of $M \propto r^{2}$ if the correlation in Figure 7 was purely due to the underlying distribution of source distances. We obtain a power-law index of 1.6 from our line of best fit in $M-R$ space, indicating that this is not the case.

The derivation of mass for each source also allows the maser sources to be placed in $M-\Sigma$ space. The locations of protostellar sample and the maser sources are plotted in Figure 8 , and the thresholds of $\Sigma=0.1$ and $1.0 \mathrm{~g} \mathrm{~cm}^{-2}$ are also shown. As stated in Section 5.1, nearly all maser sources (94 per cent) and the majority of protostellar sources (80 per cent) fall above the revised threshold of $0.1 \mathrm{~g} \mathrm{~cm}^{-2}$ for magnetised regions to form massive stars proposed by Tan et al. (2014). Traficante et al. (2018) find an approximate threshold of $0.12 \mathrm{~g} \mathrm{~cm}^{-2}$ from studies of MYSOs dark at $70 \mu \mathrm{m}$, and therefore mostly starless. Our approximate lower limit of $0.08 \mathrm{~g} \mathrm{~cm}^{-2}$ for a clump to host a class II methanol maser, and a massive protostar to sustain it, is remarkably similar to these estimates despite vast differences in our methods.

\subsection{Luminosity}

Calculated from the tabulated fluxes from each source rather than fitting (Section 4.2), the FIR luminosity distributions are shown in Figure 9, in addition to the specific luminosities at 70,160 and $250 \mu \mathrm{m}$ in Figure 10. These luminosities are given by $L_{\lambda}=4 \pi f_{\lambda} d^{2}$ for sources with distance, with no correction for bandwidth as we are only making a comparison between Hi-GAL fluxes. As described in Section 2.1, the distances assigned to each MMB source are compiled from HI self-absorption studies and supplemented with values from previous literature using techniques such as astrometry (Green \& McClure-Griffiths 2011; Green et al. 2017; Reid et al. 2016). 


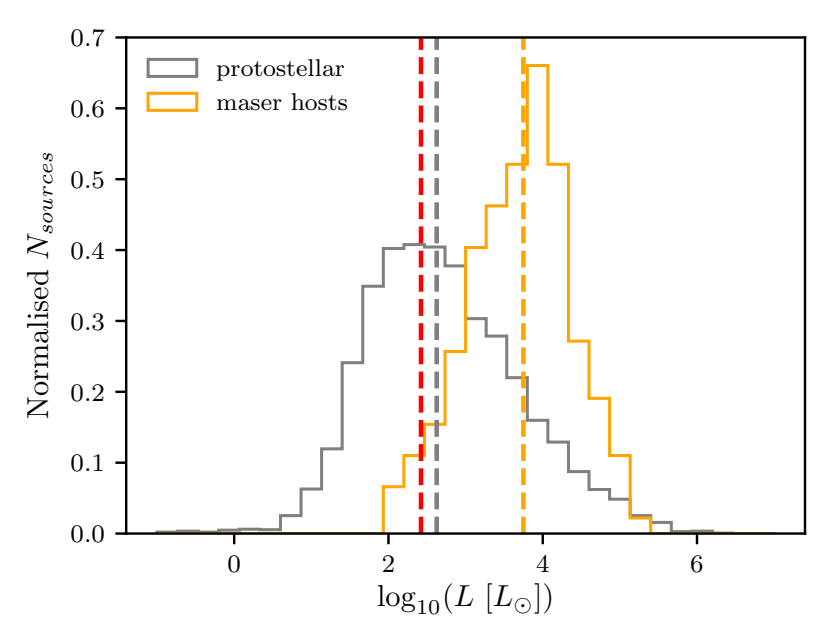

Figure 9. The far-infrared luminosity distributions of sources that host a methanol maser (orange) and the total Galactic population of clumps with high-mass protostars (grey). The maser sources have a median luminosity of $5617 \mathrm{~L}_{\odot}$, with a $2 \sigma$ lower bound of $264 \mathrm{~L}_{\odot}$ (red). The median luminosity of the protostellar sources is $422 \mathrm{~L}_{\odot}$. It should be noted that for the maser sources, the lowest luminosity sources have large distance uncertainties.

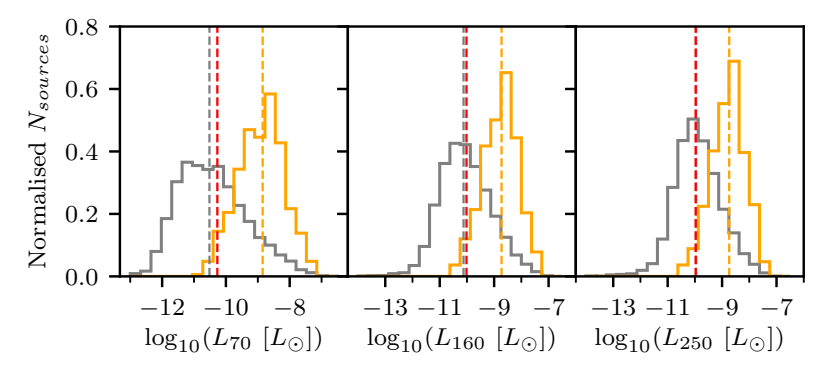

Figure 10. The luminosities at $70 \mu \mathrm{m}, 160 \mu \mathrm{m}$ and $250 \mu \mathrm{m}$ for the maser (orange) and general population of protostellar MYSOs in the Galaxy (grey), calculated from the flux in a given Hi-GAL band multiplied by $4 \pi d^{2}$ where $d$ is the distance to the source. From left to right: the maser sources have medians of $L_{70}, L_{160}$ and $L_{250}$ of $1.42 \times 10^{-9} \mathrm{~L}_{\odot}, 1.87 \times 10^{-9} \mathrm{~L}_{\odot}$ and $1.83 \times 10^{-9} \mathrm{~L}_{\odot}$ respectively. The corresponding medians for the protostellar sources are $3.05 \times 10^{-11} \mathrm{~L}_{\odot}, 7.50 \times 10^{-11} \mathrm{~L}_{\odot}$ and $1.11 \times 10^{-10} \mathrm{~L}_{\odot}$, and the lower thresholds on each luminosity for a source to host a methanol maser (red) are $5.39 \times 10^{-11} \mathrm{~L}_{\odot}, 9.64 \times 10^{-11} \mathrm{~L}_{\odot}$ and $1.07 \times 10^{-10} \mathrm{~L}_{\odot}$.

As high-mass protostellar objects are not expected to have far-infrared luminosities $\lesssim 100 \mathrm{~L}_{\odot}$, all 16 maser-hosting clumps with a derived luminosity below this threshold have been checked for reliability. Sources occupying the lowest luminosity region of the sample tend to have small heliocentric distances, and therefore a small component of their observed velocity relative to the local standard of rest is due to Galactic rotation. This results in a large percentage uncertainty on the inferred kinematic distance for these sources relative to the majority of the sample, in addition to as yet untested accuracy of the applied Reid et al. (2016) method for calculating distances in the Southern Galactic plane due to the lack of parallax measurements for comparison. The lu- minosities of these sources are therefore poorly constrained, with the distance distribution of low-luminosity objects in the sample discussed further in Section 6.2.

Twelve of the 16 sources are found at near distances $(\sim 1-2 \mathrm{kpc})$. The uncertainty in the distance to the maser is sufficient to account for the low luminosity, with each source consistent with a luminosity of $>100 \mathrm{~L}_{\odot}$ within $3 \sigma$. These 12 sources are therefore excluded from further distancedependent analysis. The remaining 4 sources have very low derived luminosities of $<20 \mathrm{~L}_{\odot}$, and in conjunction with other unusual derived properties. These sources are also excluded as the SEDs are unrealistic given the expected approximate range of source properties (false associations, unreliable flux measurement etc.) despite being physically valid.

The median value of $L_{\mathrm{FIR}}$ is found to be $5617 \mathrm{~L}_{\odot}$ for the maser sources, with a $2 \sigma$ lower bound of $264 \mathrm{~L}_{\odot}$ used to define the minimum maser-hosting clump luminosity. The corresponding medians for $L_{70}, L_{160}$ and $L_{250}$ are $1.42 \times 10^{-9} \mathrm{~L}_{\odot}$, $1.87 \times 10^{-9} \mathrm{~L}_{\odot}$ and $1.83 \times 10^{-9} \mathrm{~L}_{\odot}$, with thresholds set at $5.39 \times 10^{-11} \mathrm{~L}_{\odot}, 9.64 \times 10^{-11} \mathrm{~L}_{\odot}$ and $1.07 \times 10^{-10} \mathrm{~L}_{\odot}$ respectively.

The protostellar sample has a median bolometric luminosity of $422 \mathrm{~L}_{\odot}$ with only 58 per cent of the sample falling above the $264 \mathrm{~L}_{\odot}$ threshold set from the FIR estimate for the maser sample. Although the factor of 2 over-estimation in luminosity for the protostellar sources relative to the maser sources is small when compared to the offset in the median values, this further enhances the difference in average luminosity between the two populations. In order of increasing wavelength, the matching median values of specific luminosity are $3.05 \times 10^{-11}, 7.50 \times 10^{-11}$ and $1.11 \times 10^{-10} \mathrm{~L}_{\odot}$ and the thresholds set on specific luminosities are similarly strong, with 41,46 and 51 per cent of the protostellar sample meeting the assumed requirement to host a methanol maser in $L_{70}, L_{160}$ and $L_{250}$ respectively.

\subsection{Distance dependence and luminosity-to-mass ratio}

As shown in Figure 2, the separation between sources at the near and far heliocentric distances about Galactic centre is relatively distinct, and significant bimodality is observed in subsequent parameters such as radius. The catalogued compact sources visible with Hi-GAL are selected on their angular scale, which corresponds to different selection criteria on physical radius for the populations of objects at the near and far sides of the Galaxy. We are therefore selecting two different populations of objects as the sources at greater distances are selected to be larger. Comparable objects at nearby distances may be resolved into several objects, and similarly the population of small clumps hosting methanol masers on the far side of Galaxy will also not be detected. Although nearly all masers have a counterpart detected in at least one wavelength, we do not detect the full population of $6.7 \mathrm{GHz}$ as multiwavelength sources with $\mathrm{Hi}-\mathrm{GAL}$ as 270 lack a detection all required bands.

Separating the sources into 'near' and 'far' distances about the Galactic centre at $8.0 \mathrm{kpc}$, we select 256 and 255 maser sources at the near and far distances respectively. For the protostellar objects, the split is skewed towards near distances, finding 4229 at $<8 \mathrm{kpc}$ and 1268 at $>8 \mathrm{kpc}$. Ta- 

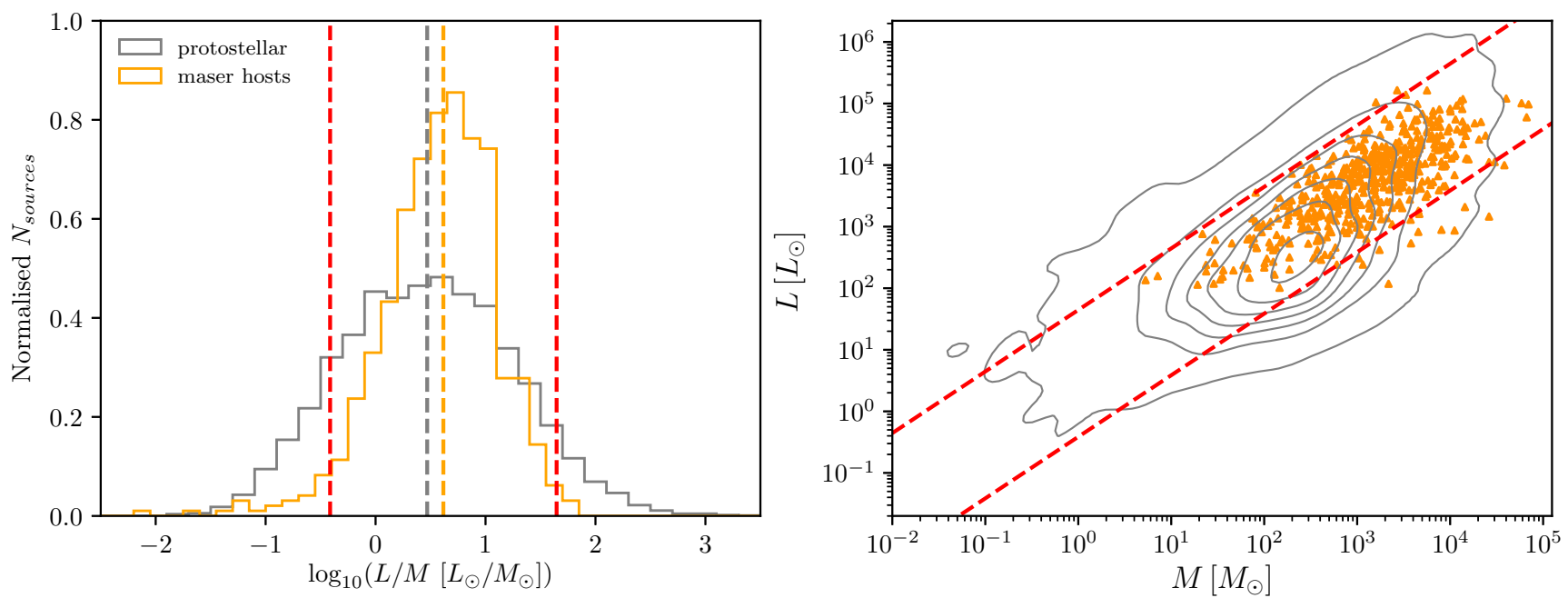

Figure 11. The distribution of luminosity-to-mass ratio as derived from the far-infrared bolometric luminosity and the mass from SED fitting for each source in the maser (orange, $L_{F I R}$ ) and protostellar (grey, $L_{b o l}$ ) sample. Left: The distributions of the two samples, with the medians of $4.1 \mathrm{~L}_{\odot} / \mathrm{M}_{\odot}$ (maser) and $2.9 \mathrm{~L}_{\odot} / \mathrm{M}_{\odot}$ (protostellar) marked and thresholds taken to be the minimum and maximum $L / M$ to host a methanol maser marked in red at $0.39 \mathrm{~L}_{\odot} / \mathrm{M}_{\odot}$ and $44.32 \mathrm{~L}_{\odot} / \mathrm{M}_{\odot}$ respectively. Right: The position of the sources in each sample in $L-M$ space, with the same assumed thresholds to host a maser marked as red lines. The maser hosts are marked in orange, with the grey contours showing the distribution of the protostellar sources. The contour levels are at intervals of $15 \%$ of the peak density in L-M space, with the lowest contour at $1 \%$ and the highest contour at $90 \%$.

Table 3. Median properties of the maser hosts and protostellar clumps at heliocentric distances $<8 \mathrm{kpc}$ (near) and $>8 \mathrm{kpc}$ (far). The values in brackets are the standard Gaussian errors.

\begin{tabular}{lcccc}
\hline Property & $\begin{array}{c}\text { Maser } \\
\text { Near }\end{array}$ & Far & Protostellar \\
& Near & Far \\
\hline$d[\mathrm{kpc}]$ & $3.8(1.5)$ & $11.4(1.8)$ & $3.5(1.5)$ & $10.7(2.1)$ \\
$r[\mathrm{pc}]$ & $0.2(0.1)$ & $0.6(0.1)$ & $0.2(0.1)$ & $0.5(0.2)$ \\
$\log _{10}\left(M\left[\mathrm{M}_{\odot}\right]\right)$ & $2.8(0.6)$ & $3.5(0.5)$ & $2.1(0.8)$ & $3.0(0.6)$ \\
$\log _{10}\left(L_{\mathrm{FIR}}\left[\mathrm{L}_{\odot}\right]\right)$ & $3.4(0.6)$ & $4.0(0.5)$ & $2.3(0.9)$ & $3.6(0.8)$ \\
$\log _{10}\left(L / M\left[\mathrm{~L}_{\odot} / \mathrm{M}_{\odot}\right]\right)$ & $0.7(0.5)$ & $0.6(0.5)$ & $0.4(0.8)$ & $0.7(0.7)$ \\
\hline
\end{tabular}

ble 3 reports the typical distance-dependent properties for the 'near' and 'far' objects for the maser and protostellar objects. As expected, sources at the far distances are found to have significantly larger radii than nearby sources, and are a factor of $\sim 3$ larger. Similarly, an increase in median mass and luminosity is also found. In many cases, the uncertainty on the distance to a source is also significant and corresponds to an increased uncertainty in parameters calculated from the measured distance relative to parameters that are not.

The primary focus of this work is to characterise the evolutionary status of MYSOs hosting class II methanol masers, and we are therefore concerned with the evolutionary bias between the near and far sources. Mass-luminosity diagrams are a common diagnostic used to place an MYSO along its evolutionary sequence, with the overall trend in the space for a sample characterised by the luminosity-tomass ratio, $L / M$. Again, we emphasise that this work defines this as the ratio between FIR luminosity and mass, whereas discussions in other work including Elia et al. (2017) discuss the luminosity-to-mass ratio using bolometric luminos- ity. Although luminosity and mass are both calculated from measured distance, this parameter is not (as both are proportional to $d^{2}$ ), giving an estimate of the evolutionary status of a clump without introducing significant uncertainties, and associated correlations, through the use of a distance value. Shown in the last row of Table 3 , the median $L / M$ for sources at the near and far distances are very similar for the maser sources. We therefore conclude that although we are sampling different sized clumps with heliocentric distances either side of Galactic centre, the two populations maser hosts are of similar evolutionary status and we do not separate them when discussing parameters that are not dependent on the scale of a clump.

Shown in the right panel Figure 11, the methanol maser hosts occupy a band within the protostellar regime, towards the upper end, in a mass-luminosity diagram. Although the median $L / M$ of the maser sample of 4.1 is close to the protostellar sample median of 2.9 , the resulting distribution is significantly narrower for the maser host clumps, as shown in the left panel of Figure 11. Both lower and upper limits are therefore set on the $L / M$ value of a clump that can host a methanol maser, at $0.39 \mathrm{~L}_{\odot} / \mathrm{M}_{\odot}$ and $44.32 \mathrm{~L}_{\odot} / \mathrm{M}_{\odot}$ respectively, marked in red on both panels. This threshold is low, given that Molinari et al. (2016b) find the expected range of $L / M$ for protostellar sources to be $1 \lesssim L / M \lesssim 10$. However, our estimate of $L / M$ is not directly comparable as we use the far-infrared luminosity only, rather than the bolometric luminosity over all frequencies. Combining the factors of 2 (due to the definition of luminosity, Section 4.2) and 1.4 (choice of reference dust mass opacity, Section 5.1) results in a factor of $\sim 3$ lower $L / M$ for the maser sources compared with the protostellar sample from Elia et al. (2017). Accounting for this factor enhances the offset in median between the 


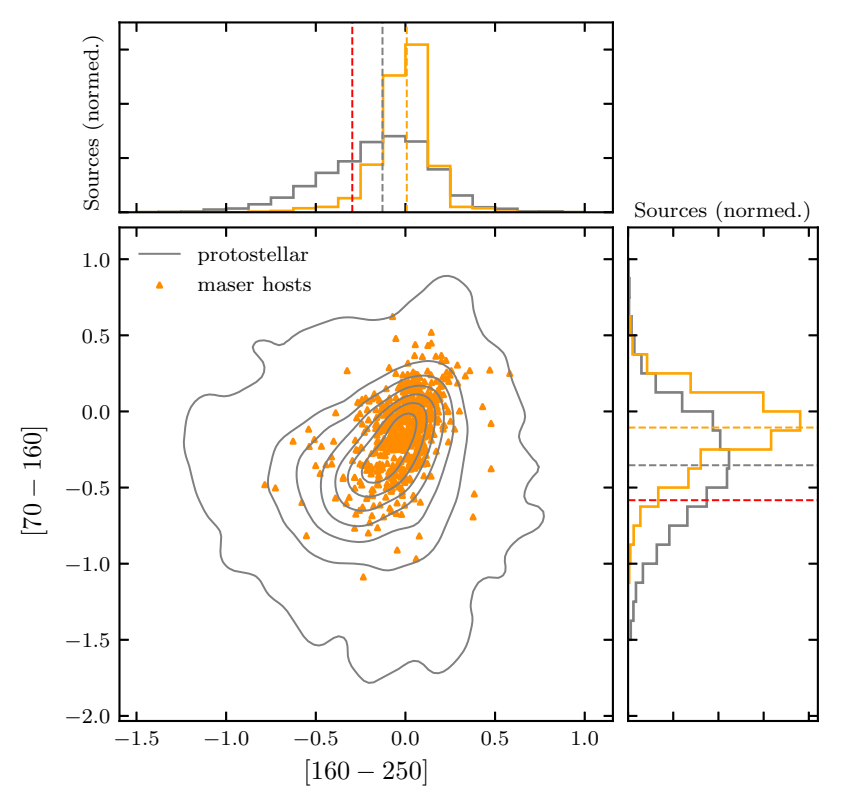

Figure 12. Colour-colour diagram showing the [70-160] $\mu \mathrm{m}$ colour against the [160-250] $\mu \mathrm{m}$ colour for the protostellar (grey contours, from lowest to highest density in colour-colour space: $1 \%, 15 \%, 30 \%, 45 \% 60 \%, 75 \%, 90 \%)$ and maser host (orange) sources respectively. The normalised distributions of each colour are shown to the right of and above the scatter plot respectively, with the medians marked in addition to the lower limit on each colour taken as a requirement to host a methanol maser.

two samples, strengthening the conclusion that the maser sources are, on average, found at greater $L / M$.

\subsection{Colours}

The temperature derived from fitting to the far-infrared SED will primarily trace the cold protostellar envelope of a clump rather than the temperature of the warm embedded protostar responsible for the excess of flux over the greybody value at $70 \mu \mathrm{m}$. The colour of a source, defined as the logarithmic ratio of two fluxes, can characterise the slope of an SED and therefore the temperature of an object without full knowledge of the form of the spectral energy distribution. Figure 12 displays the colour-colour diagram for the clumps from the [160-250] $\mu \mathrm{m}$ colour and the [70-160] $\mu \mathrm{m}$ colour, with the axis histograms showing the distribution of each. The $70 \mu \mathrm{m}$ flux of the maser clumps is on average stronger relative to the $160 \mu \mathrm{m}$ flux than the protostellar objects, resulting in a more positive [70-160] $\mu \mathrm{m}$ colour, as is the ratio of $160 \mu \mathrm{m}$ to $250 \mu \mathrm{m}$ flux. This implies that the warm component responsible for the emission at the shortest wavelengths is more significant relative to the total thermal emission from all components seen at longer wavelengths. A similar result is obtained when considering the [70-250] $\mu \mathrm{m}$ colour, with the medians and thresholds of the source colours for each sample summarised in Table 4.

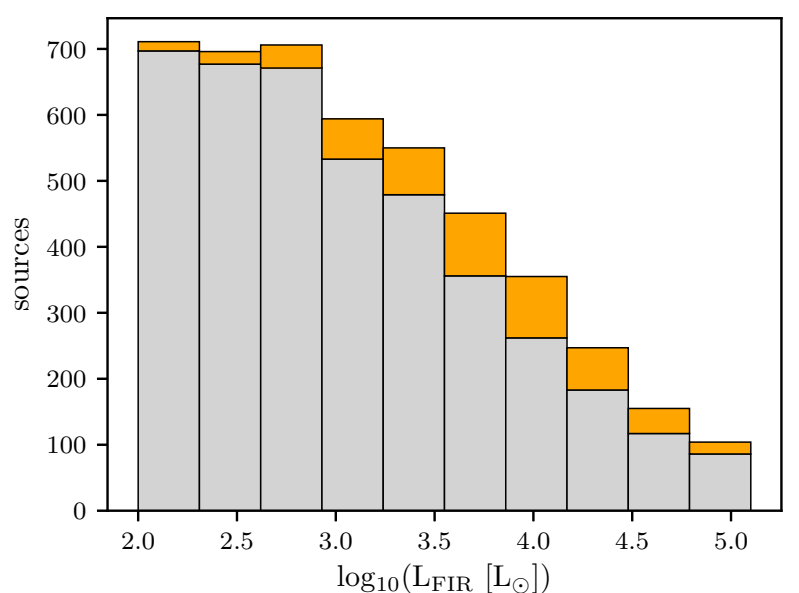

Figure 13. Stacked histogram of the protostellar sources (grey) and maser sources (orange) as a function of far-infrared luminosity.

\subsection{Completeness}

We find the majority of methanol masers to be associated with compact dust emission with over $95 \%$ of the maser sources detected in at least one wavelength of Hi-GAL. However, to associate a maser with a Hi-GAL source we require a detection in four Hi-GAL bands (70, 160, 250 and $350 \mu \mathrm{m})$ and $27 \%$ do not satisfy this requirement. Also, $18 \%$ of the maser sources are excluded from the analysis as they do not have a distance determined.

In the following analysis of the maser sources (Section 8.2), we derive a relation between the FIR clump luminosity and the integrated luminosity of its $6.7 \mathrm{GHz}$ maser. The line of best fit to the medians $L_{6.7}\left[\mathrm{Jy} \mathrm{km} \mathrm{s}^{-1} \mathrm{kpc}^{2}\right]$ of $\log _{10}\left(L_{6.7}\right)=(0.8 \pm 0.1) \log _{10}\left(L_{F I R}\right)+(0.4 \pm 0.2)$. For a given $L_{F I R}$ in $\mathrm{L}_{\odot}$, the average scatter in $6.7 \mathrm{GHz}$ luminosity is $10^{0.6}$. For a clump of $100 \mathrm{~L}_{\odot}$, the expected maser luminosity from this relation is $100 \mathrm{Jy} \mathrm{km} \mathrm{s}^{-1} \mathrm{kpc}^{2}$, decreasing to $10^{1.4} \mathrm{Jy} \mathrm{km} \mathrm{s}^{-1} \mathrm{kpc}^{2}$ when considering the quoted scatter. At $2 \mathrm{kpc}$, these masers would have integrated fluxes of $2.0 \mathrm{Jy} \mathrm{km} \mathrm{s}^{-1}$ and $0.5 \mathrm{Jy} \mathrm{km} \mathrm{s}^{-1}$ respectively.

Green et al. (2017) give the luminosity sensitivity of the MMB as $0.15 \mathrm{Jy} \mathrm{km} \mathrm{s}^{-1}$, assuming a maser line to span 3 spectral channels each with a sensitivity of $0.51 \mathrm{Jy}$. Both the average and lower bound on expected luminosity of a methanol maser in a $100 \mathrm{~L}_{\odot}$ clump are therefore detectable to greater than $3 \sigma$ with the MMB sensitivity out to a distance of $2.7 \mathrm{kpc}$ for a maser of average luminosity in a clump of $100 \mathrm{~L}_{\odot}$, so we consider the MMB to be complete out to this limit.

The completeness of the Hi-GAL catalogue is harder to assess. Elia et al. (2017) find the $90 \%$ mass completeness limit to be better than $10^{1.45} \mathrm{M}_{\odot}$ for all objects closer than $2.7 \mathrm{kpc}$. Adopting a minimum luminosity to mass ratio of -0.1 (Fig. 11), this mass limit corresponds to a luminosity limit of $22 \mathrm{~L}_{\odot}$, significantly below the lowest luminosity of one of the maser sources, $100 \mathrm{~L}_{\odot}$, suggesting that the completeness of the Hi-GAL catalogue is not an issue for our study. The dominant issue limiting the completeness of the 
protostellar sources is the large fraction missing a reliable distance, with only one in four sources assigned a reliable distance.

Figure 13 displays the number of maser sources relative to the number of protostellar sources as a function of luminosity for the entire sample. This shows a distinct lack of maser sources relative to the number of protostellar sources at low luminosities. Comparing the number of sources detected within $2.7 \mathrm{kpc}$ only (where the MMB is complete for low luminosity sources of $\sim 100 \mathrm{~L}_{\odot}$ ) we find 3 maser sources with luminosity between 80 and $120 \mathrm{~L}_{\odot}$ and 92 protostellar sources, a ratio of 1:31. Within the same distance, there are 24 maser sources with luminosities $>10^{3} \mathrm{~L} \odot$ and 64 protostellar sources, a ratio 1:3. This is considerably larger than for the lower luminosity sources and demonstrates that the paucity of low luminosity maser sources is not a result of incompleteness. Note that these values have not been corrected for the completeness of the Hi-GAL sample. However, this should not affect the conclusion as the Hi-GAL completeness is dominated by sources with poorly defined distances.

A final potential consideration for the completeness of maser studies is the effect of beaming, as the amplitude of emission from a maser 'spot' can be a strong function of the direction to the line of sight. Strong class II methanol masers have several spectral components or spots so that statistically the beaming can be assumed to be homogeneous. However, for lower luminosity maser sources with fewer components, beaming may have a more significant, but difficult to quantify, effect on the source counts.

Recent VLBI studies (e.g. Bartkiewicz et al. 2009, 2014, 2016; Pandian et al. 2011; Fujisawa et al. 2014) have found $6.7 \mathrm{GHz}$ masers with a range of morphologies consistent with several different physical origins of the maser emission within a protostellar clump. One of these maser morphologies is a ring structure which has been used to suggest that some methanol masers arise in the disks associated with young high-mass stars. Naively, it might be thought that masers associated with some of these structures, such as disks, for example, might have a limited range of preferred beaming angles. However, VLBI observations have shown ring-like structures at a range of inclination angles (e.g. Moscadelli et al. 2010; Bartkiewicz et al. 2016; Sanna et al. 2017). Therefore it is unlikely that there is a consistent preferred beaming direction within most maser sites and we consequently assume isotropic emission.

\subsection{Summary of results}

The above analysis finds, on average, that protostellar clumps hosting class II methanol masers are hotter, more luminous in both bolometric and single wavelength luminosities, more massive and at greater mass surface densities than the general Galactic population of protostars, although they are at a comparable luminosity-to-mass ratio with a narrower distribution of values. The median properties and standard deviations for all properties are listed in Table 4 for the maser hosting and protostellar objects, alongside the threshold set on each property as the clump requirements to host a methanol maser.

For comparison, the values obtained by Urquhart et al. (2015) in their study of the ATLASGAL $870 \mu \mathrm{m}$ dust clumps associated with class II methanol masers have also been in- cluded in this table. For their derivation of clump properties, a single-temperature greybody with fixed $T=20 \mathrm{~K}$ was assumed. As for the protostellar objects, Urquhart et al. (2015) use a difference dust mass opacity which results in systematically increasing our masses by a factor of $1.8\left(\sim 10^{0.3}\right)$ relative to their results. The average mass derived for maser host clumps here is slightly smaller than the value derived by Urquhart et al. (2015).

Converting their average $\mathrm{H}_{2}$ column density of $N_{\mathrm{H}_{2}}=$ $10^{22.74} \mathrm{~cm}^{-2}$ to a mass surface density using

$\Sigma=\frac{71}{\pi} \mathrm{M}_{\odot} \mathrm{pc}^{-2}\left[\frac{N_{\mathrm{H}_{2}}}{10^{21} \mathrm{~g} \mathrm{~cm}^{-2}}\right]$

and a conversion factor of $4800 \mathrm{M}_{\odot} \mathrm{pc}^{-2}=1 \mathrm{~g} \mathrm{~cm}^{-2}$ (Kauffmann et al. 2010), we obtain a mass surface density of $\log _{10}\left(\Sigma\left[\mathrm{g} \mathrm{cm}^{-2}\right]\right)$ of $-0.59 \pm 0.51$. Although both mass and mass surface density are consistent within errors, a direct comparison of the two results is not particularly meaningful as the significantly lower value can be mostly attributed to the relatively larger radius averaged over each clump (average size $0.93 \mathrm{pc}$ in Urquhart et al. 2015, compared with $0.42 \mathrm{pc}$ in the current work). The assumption of $T=20 \mathrm{~K}$ will also modify the results for individual sources, but as this is comparable to the median temperature for maser hosts of $19.5 \mathrm{~K}$, it is unlikely the cause of a systematic offset in mass surface density between the two studies.

\section{REQUIREMENTS TO HOST A METHANOL MASER}

From the results presented in Section 5, the higher temperature and stronger flux at $70 \mu \mathrm{m}$ that we find for maserbearing clumps particularly highlights that the differences in properties may be due to evolutionary differences. The distinct band occupied by the methanol maser hosts within the total $L / M$ range of the protostellar sample may imply that methanol masers are associated with a shorter-lived phase of protostellar evolution, appearing once an embedded protostar is sufficiently evolved to sustain the maser pumping but dispersing before the protostar reaches its final mass, possibly a result of the destruction of methanol in the circumstellar environment.

The thresholds set throughout Section 5 on each property are used to identify sources capable of hosting a class II methanol maser based on the FIR host clump properties. Applying each of the thresholds and removing clumps associated with methanol masers from the protostellar sample, which are subject to the selection criteria stated at the start of Section 5, 896 (out of 5497, 16 per cent) of the protostellar clumps are found to satisfy all requirements yet have no detected $6.7 \mathrm{GHz}$ maser within them. With 731 of methanol maser host clumps detected as protostellar sources with Hi-GAL, and 647 with SEDs appropriate for analysis, this implies that class II methanol masers are detected towards only approximately half of the clumps which, based on the properties discussed here, could host a maser.

We investigate the differences between maser hosting and this subset of non-maser clumps further by first searching for segregation between the types of objects in any combination of FIR properties, followed by a comparison of these two types of objects for sources of similar luminosities. The 


\begin{tabular}{|c|c|c|c|c|c|c|c|}
\hline \multirow[t]{2}{*}{ Property } & \multicolumn{2}{|c|}{ Maser } & \multicolumn{2}{|c|}{ Protostellar } & \multirow[t]{2}{*}{ Threshold } & \multicolumn{2}{|c|}{ Urquhart et al. (2015) } \\
\hline & Median & Std. dev. & Median & Std. dev. & & Median & Std. dev. \\
\hline$T[\mathrm{~K}]$ & 19.5 & 4.6 & 15.3 & 5.6 & 12.5 & $(20)$ & - \\
\hline $\log _{10}\left(\Sigma\left[\mathrm{g} \mathrm{cm}^{-2}\right]\right)$ & -0.12 & 0.50 & -0.47 & 0.60 & -1.12 & -0.59 & 0.51 \\
\hline$r[\mathrm{pc}]^{\dagger}$ & 0.42 & 0.23 & 0.19 & 0.21 & 0.11 & 0.93 & 0.96 \\
\hline $\log _{10}\left(M\left[\mathrm{M}_{\odot}\right]\right)^{\dagger}$ & 3.16 & 0.66 & 2.31 & 0.82 & 1.84 & 3.28 & 0.67 \\
\hline $\log _{10}\left(L_{\mathrm{FIR}}\left[\mathrm{L}_{\odot}\right]\right)^{\dagger}$ & 3.75 & 0.66 & 2.63 & 0.99 & 2.42 & - & - \\
\hline $\left.\log _{10}\left(L_{70} \mathrm{~L}_{\odot}\right]\right)$ & -8.85 & 0.71 & -10.52 & 1.08 & -10.27 & - & - \\
\hline $\left.\log _{10}\left(L_{160} \mathrm{~L}_{\odot}\right]\right)$ & -8.72 & 0.64 & -10.12 & 0.95 & -10.02 & - & - \\
\hline $\left.\log _{10}\left(\begin{array}{ll}L_{250} & \mathrm{~L}_{\odot}\end{array}\right]\right)$ & -8.74 & 0.62 & -9.95 & 0.86 & -9.97 & - & - \\
\hline $\log _{10}\left(L / M\left[\mathrm{~L}_{\odot} / \mathrm{M}_{\odot}\right]\right)^{\dagger}$ & 0.62 & 0.52 & 0.47 & 0.77 & $-0.41,1.65$ & - & - \\
\hline [70-160] colour & -0.11 & 0.24 & -0.35 & 0.37 & -0.58 & - & - \\
\hline [160-250] colour & 0.01 & 0.15 & -0.13 & 0.30 & -0.30 & - & - \\
\hline [70-250] colour & -0.11 & 0.33 & -0.52 & 0.52 & -0.77 & - & - \\
\hline
\end{tabular}

Table 4. Summary of all properties derived from the Hi-GAL fluxes for the clumps hosting $6.7 \mathrm{GHz}$ class II methanol masers, and the corresponding properties for the general Galactic protostellar population. Following the median and standard deviation of a property for each set of objects, the lower threshold set as the clump requirement to host a methanol maser given by the 2- $\sigma$ width of the maser host distribution are given. For $L / M$, an upper threshold is also given. The final two columns give the values derived from dust emission towards $6.7 \mathrm{GHz}$ host clumps from Urquhart et al. (2015), where a dust temperature of $20 \mathrm{~K}$ was assumed for all clumps and we have converted the reported average column density to a mass surface density by Equation 5 .

$\dagger$ These properties are given for sources at near $(<8 \mathrm{kpc})$ and far $(>8 \mathrm{kpc})$ distances separately in Table 3.

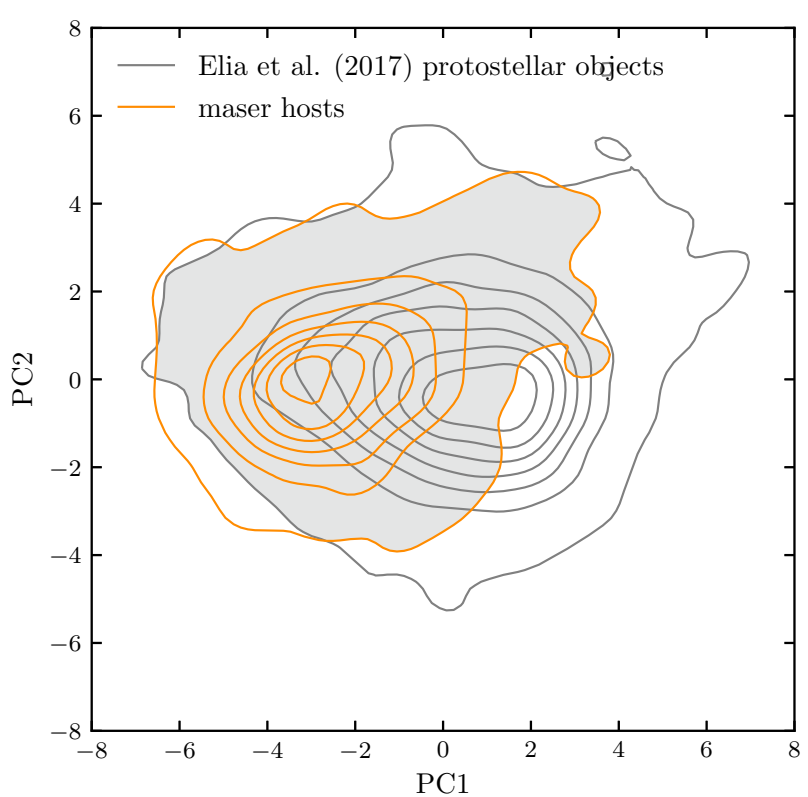

Figure 14. The distributions of maser (orange contours) and non-maser (grey contours) sources in the plane of the first and second principal component. The shaded overlap indicates the 896 sources maser and non-maser sources with identical properties in the 8-dimensional infrared parameter space defined by the input to the PCA. For each set of sources, the contour levels are at intervals of $15 \%$ between $1 \%$ and $90 \%$ of the peak density in PC1-PC2 space.

findings of this analysis and possible underlying physical causes for sources to lack a detected $6.7 \mathrm{GHz}$ methanol maser are then discussed in Section 6.3.

\subsection{Principal component analysis}

When studying objects defined by a large number of properties (high-dimensionality), identifying trends between every possible combination of parameters is an incredibly lengthy task. Although we have presented the individual properties of the methanol maser host clumps and a select few combinations of these in Section 5, this is not a full investigation of mutual trends between the properties. Failing to consider all combinations may result in missed significant differences between maser and non-maser protostellar objects, such as finding maser objects to be at greater mass and luminosity individually but finding them to be restricted to a narrow middle band in luminosity-to-mass ratio.

Principal component analysis (PCA) provides a method with which to reduce the dimensionality of the data by defining new variables along mutual trends in parameters, so that we only need to consider a few variables to fully define the clumps. In addition to identifying trends in several parameters within a set of data, PCA may also be used to identify differences in two sets of objects if they are sufficiently offset in the original parameter space. Due to the large overlap in each property seen in Section 5, we do not expect to find clear separation in the new parameter space but instead define a region of overlap between the two samples to identify a sample of objects matched in all properties to the methanol maser hosts, but lacking a maser.

Both the maser and non-maser protostellar clumps are taken to be defined by eight properties: $L_{70}, L_{250}$, [70 - 160] colour, [70 - 250] colour, $L_{\mathrm{FIR}}, M, r$ and $T$. These are used as the original variables, chosen as they are both intrinsic properties and not necessarily related through combinations of each other. PCA is a linear technique and to allow for power-law dependencies between parameters, logarithms of $L_{70}, L_{250}, L_{\mathrm{FIR}}, M$ and $r$ were taken prior to input to the PCA. We find only the first two principal components, PC1 and $\mathrm{PC} 2$, to be significant trends. PC1 represents a mutual increase in $L_{\mathrm{FIR}}, L_{70}, L_{250} M$ and $r$ and therefore represents the trend in observed parameters with the scale of a clump. 
An independent trend in increasing [70 - 160] and [70 - 250] colours and temperature with decreasing mass and radius forms $\mathrm{PC} 2$, and we interpret this as a likely evolutionary trend. Further discussion of the individual components and techniques is included in Appendix A.

Figure 14 displays the first and second principal components plotted against each other with points categorised by sample, showing clear separation in the peak location of the maser and non-maser objects, although there is significant overlap. The non-maser objects falling within the contours occupied by the maser sources are the 896 satisfying the conditions to host a methanol maser in all infrared parameters. This large overlap (an approximately equal numbers of maser and non-maser sources) confirms that whilst there are significant statistical differences between the populations of maser and non-maser protostellar objects, we cannot easily identify whether a MYSO will host a methanol maser through any combination of the derived infrared parameter.

\subsection{Luminosity matched comparison}

Although 896 of the non-maser sources meet all individual requirements on each IR-derived property to host a methanol maser, a comparison of these sources against maser sources of the same luminosity excludes the scatter introduced by considering objects of vastly different size, evolutionary state or energy output. This allows us to examine any subtle differences between protostellar clumps that are matched in their IR properties to methanol maser hosts, but lack a detection with the MMB survey.

The sources are split into three bins in $L_{F I R}: 10^{2}-$ $10^{3} \mathrm{~L}_{\odot}, 10^{3}-10^{4} \mathrm{~L}_{\odot}$ and $10^{4}-10^{5.5} \mathrm{~L}_{\odot}$. These bins were chosen to include a sufficient number of sources in each bin to determine differences with significance and to include the majority of the high-luminosity tail. The distributions of luminosity within each bin are found to be identical between the two types of object, therefore excluding any luminosity biases in each bin as a possible cause for any other property differences. However, the distance distribution is shown in Figure 15 for the MMB sources in each luminosity bin and the majority of sources in the low-luminosity bin are found at relatively small distances. As discussed in Section 5.3, small distances carry a much greater uncertainty and the distance-dependent properties of these sources, such as luminosity, are less reliable compared to sources at greater distances. The average properties derived here for the sources in the lowest luminosity bin are therefore unlikely to give an accurate characterisation of methanol maser sources with luminosities $<1000 \mathrm{~L}_{\odot}$.

The restricted non-maser sample is compared against the maser sources in each other IR property with the median values shown in Table 5 . Unlike the previous comparisons shown, the differences in the median value of each property between the non-maser and maser sources are less distinct in comparison to the offsets reported in Section 5. The KS test p-values between the two categories of objects are also given to determine whether a difference in median likely corresponds to a difference in the underlying distribution.

For the reasons above, we consider the statistics for the second and third luminosity bins to best reflect the physical conditions of each sample. The most notable difference between the maser and protostellar objects, both in difference

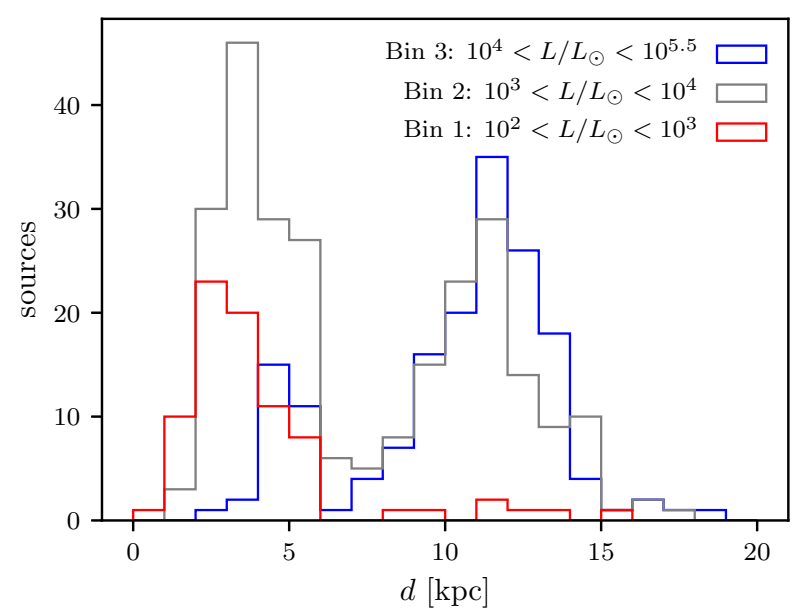

Figure 15. Heliocentric distance distributions of the MMB sources with far-infrared clump luminosities between $10^{2}-10^{3} \mathrm{~L}_{\odot}$, $10^{3}-10^{4} \mathrm{~L}_{\odot}$ and $10^{4}-10^{5.5} \mathrm{~L}_{\odot}$ for bins 1 (red), 2 (grey) and 3 (blue) respectively.

in the median and statistical significance, is seen in $70 \mu \mathrm{m}$ luminosity with a factor of 3.2 increase in the median for maser hosts over the protostellar objects in the mid luminosity bin between $10^{3}$ and $10^{4} \mathrm{~L} \odot$. We also see this for the other specific luminosities at 160 and $250 \mu \mathrm{m}$, although the contrast between the two median values is slightly smaller. As noted in Section 4.2, this may be due to the inclusion of additional wavelengths during the luminosity calculation for the protostellar sample. However, the factor of two due to this is not sufficient to account for the offset in the specific luminosities, and therefore this implies that the maser sources are stronger in these shorter wavelengths than the protostellar objects relative to their total luminosity. This trait is also reflected in the [70-160] and [70-250] colours which trace the inner colour temperature of a clump, and the overall envelope temperature although these results are less statistically significant.

\subsection{Physical interpretation}

A number of physical scenarios may lead to some clumps hosting class II methanol masers and others lacking this feature. Firstly, we discount the possibility that only the most extreme (e.g. most massive, most luminous, etc) sources may host a methanol maser as all sources fall above the thresholds on each property to host a methanol maser. Notably, we find that sources down to a threshold of $\sim 0.08 \mathrm{~g} \mathrm{~cm}^{-2}$ are capable of hosting a methanol maser and therefore a massive protostar, comparable to the threshold of approximately $0.05 \mathrm{~g} \mathrm{~cm}^{-2}$ for massive star formation found by Urquhart et al. (2018). As $6.7 \mathrm{GHz}$ methanol masers exclusively trace high-mass protostars, this suggests that this all sources above this threshold on mass surface density may form high-mass stars. We would also not expect to recover differences in typically evolutionary properties such as temperature and luminosity-to-mass ratio if this were the case. Additionally, if the two types of object are distinct and only those above some threshold in any combination of proper- 
Table 5. The median far-infrared derived properties of non-maser and maser sources when binned by luminosity to allow comparison of sources with an approximately equivalent energy output. For each bin, the median for each category is shown alongside the KS p-value for the two distributions.

\begin{tabular}{|c|c|c|c|c|c|c|c|c|c|}
\hline \multirow[t]{2}{*}{ Property } & \multicolumn{3}{|c|}{$\operatorname{Bin} 1\left(10^{2}-10^{3} \mathrm{~L}_{\odot}\right)$} & \multicolumn{3}{|c|}{$\operatorname{Bin} 2\left(10^{3}-10^{4} \mathrm{~L}_{\odot}\right)$} & \multicolumn{3}{|c|}{$\operatorname{Bin} 3\left(10^{4}-10^{5.5} \mathrm{~L}_{\odot}\right)$} \\
\hline & Non-maser & Maser & KS p-value & Non-maser & Maser & KS p-value & Non-maser & Maser & KS p-value \\
\hline No. sources & 96 & 80 & - & 567 & 258 & - & 217 & 165 & - \\
\hline $\mathrm{T}[\mathrm{K}]$ & 15.4 & 17.8 & $10^{-7}$ & 17.0 & 19.2 & $10^{-15}$ & 19.8 & 21.6 & $10^{-5}$ \\
\hline$\Sigma\left[\mathrm{g} \mathrm{cm}^{-2}\right]$ & 0.24 & 0.44 & 0.003 & 0.34 & 0.70 & $10^{-13}$ & 0.66 & 1.08 & $10^{-4}$ \\
\hline $\mathrm{M}\left[\mathrm{M}_{\odot}\right]$ & 232 & 185 & $10^{-4}$ & 465 & 991 & $10^{-17}$ & 1629 & 3873 & $10^{-12}$ \\
\hline $\mathrm{L}_{70}\left[10^{-10} \mathrm{~L}_{\odot}\right]$ & 0.74 & 1.17 & $10^{-9}$ & 2.84 & 9.11 & $10^{-34}$ & 21.72 & 56.26 & $10^{-21}$ \\
\hline $\mathrm{L}_{160}\left[10^{-10} \mathrm{~L}_{\odot}\right]$ & 1.43 & 1.61 & 0.002 & 4.48 & 12.71 & $10^{-39}$ & 25.43 & 63.73 & $10^{-32}$ \\
\hline $\mathrm{L}_{250}\left[10^{-10} \mathrm{~L}_{\odot}\right]$ & 1.74 & 2.00 & 0.020 & 4.55 & 13.74 & $10^{-39}$ & 25.13 & 58.34 & $10^{-23}$ \\
\hline$[70-160] \mu \mathrm{m}$ & -0.28 & -0.18 & $10^{-4}$ & -0.19 & -0.12 & $10^{-5}$ & -0.01 & -0.06 & 0.061 \\
\hline$[70-250] \mu \mathrm{m}$ & -0.38 & -0.24 & $10^{-4}$ & -0.19 & -0.13 & 0.009 & 0.02 & -0.01 & 0.198 \\
\hline$[160-250] \mu \mathrm{m}$ & -0.11 & -0.06 & 0.076 & -0.02 & 0.00 & 0.074 & 0.03 & 0.05 & 0.065 \\
\hline
\end{tabular}

ties host methanol masers, separation of the two categories in principal component space would be seen.

There are three scenarios that our analysis does not consider, as each would require follow-up spectral observations towards a large number of sources to confirm. A possible case is that the two types of objects are not distinct and that the non-maser sources do have masers but they are not present in the MMB catalogue due to low strengths or a beaming angle of the maser away from the line of sight. As $6.7 \mathrm{GHz}$ maser strength shows correlation with the $70 \mu \mathrm{m}$ luminosity (Section 8.2), it is possible that the non-maser sources host weak methanol masers due to their comparatively weak $70 \mu \mathrm{m}$ emission. The survey of Pandian et al. (2007) has a flux completeness limit a factor of 1.9 lower than the MMB and detected 12 additional weak maser sources which are not in the MMB survey (Breen et al. 2015). In the same longitude range the MMB survey detected 93 sources, so the weaker sources correspond to only $13 \%$ of the MMB population. This suggests that if the non-maser sources (which are similar in total number to the MMB sources) do in fact have weak masers, these masers are considerably below the MMB sensitivity, or indeed that of Pandian et al. (2007). The deeper 'piggyback' survey carried out with the MMB receiver will provide further insight on the population of weak masers (Ellingsen et al., in prep.).

The greater variability of weak $6.7 \mathrm{GHz}$ masers compared with strong masers may also contribute to weaker sources being missed by the MMB survey. However, even for weak maser sources, the typical variability is not extreme, with a measured mean variability of 36 per cent for sources of peak maser flux of < 1.5 Jy (Green et al. 2017).

Finally, due to either evolutionary effects or the properties of the natal environment of a clump, chemical differences in the circumstellar region may prevent a protostellar source from masing at $6.7 \mathrm{GHz}$. Although plausible, these scenarios cannot fully account for the differences in the distributions of IR properties that we find between the two categories.

Shown in Figure 16, we consider two evolutionary scenarios in this work that may cause the observed differences in the infrared properties of maser and non-maser sources, with evolution advancing from left to right:

1) Maser sources (middle) are at a more advanced stage of evolution and have had longer to irradiate and heat their envelopes than the protostellar objects (left).
2) The protostars in non-maser sources have cleared their immediate surroundings of dust and possibly methanol (right) and are more evolved than the maser sources (middle).

Scenario 1 is supported by our findings that maser sources have warmer envelope temperatures for a given luminosity and more prevalent emission from a warm inner region at $70 \mu \mathrm{m}$, as indicated by greater $L_{70},[70-160] \mu \mathrm{m}$ colour and [70-250] $\mu \mathrm{m}$ colour. Scenario 2 instead implies that the nonmaser sources are at a more advanced stage of evolution than maser hosts, and have sufficiently cleared either the methanol or hot dust from their surroundings required for methanol maser emission. As the $70 \mu \mathrm{m}$ emission from a MYSO originates from hot dust in the immediate protostellar environment, it is therefore also feasible that a decrease in $L_{70}$ indicates that a source is sufficiently evolved to remove this dust, rather than failing to strongly heat this dust. Removing the contribution of the warm inner component to the FIR SED of a source may also result in a lower clump-averaged temperatures recovered from fitting, and is a potential origin of the difference found in temperature between the non-maser and maser sources.

The MIR data can be used to probe which scenario is more likely correct. The emission from the central regions of a protostellar object is severely attenuated by any dusty envelope material along the line of sight. Initially, such as in the first scenario we are considering, very little of this emission is expected to escape. In the final evolutionary state shown in Figure 16, the cleared central region results in a reduced column density of material that the MIR emission must pass through to escape, resulting in brighter emission from the object. In addition to this, lines of sight through the envelope into the central regions may be cleared by outflows, also making brighter emission more likely towards objects in this evolutionary state. However, this may also result in the $8 \mu \mathrm{m}$ emission being extended, and so the source may not be included in the GLIMPSE point source catalogue. The bias towards nearer distances for the non-maser sources (Section 5) may also contribute to their $8 \mu \mathrm{m}$ counterparts being too extended to be included in the GLIMPSE point source catalogue.

Associating the non-maser sources with objects in the GLIMPSE $8 \mu \mathrm{m}$ catalogue, as done for the maser sources, 173 of the $896(19.3 \%)$ non-maser sample have an $8 \mu \mathrm{m}$ counterpart. This is notably smaller than the $48.3 \%$ of maser 


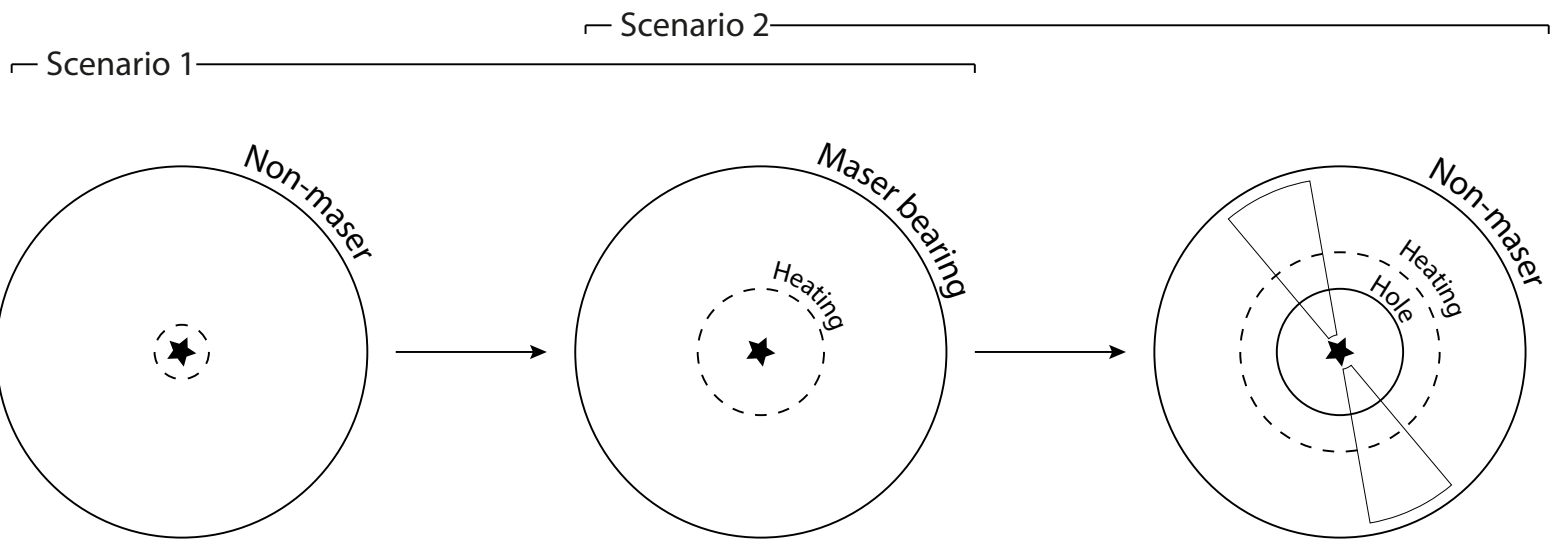

Figure 16. The two possible evolutionary scenarios considered in this work to account for the lack of detection of a methanol maser towards protostellar sources of identical infrared properties to clumps hosting methanol masers. Evolution increases from left to right, with the solid lines showing the location of the dusty envelope material and the dotted lines indicating heated regions around a central protostar (filled star). With a methanol maser source represented by the middle evolutionary state, scenario 1 considers the non-maser sources to fall at an earlier stage of evolution, and scenario 2 considers them to be at a later stage of evolution, in which a central hole in the dusty material has been created and outflows may be present (cones).

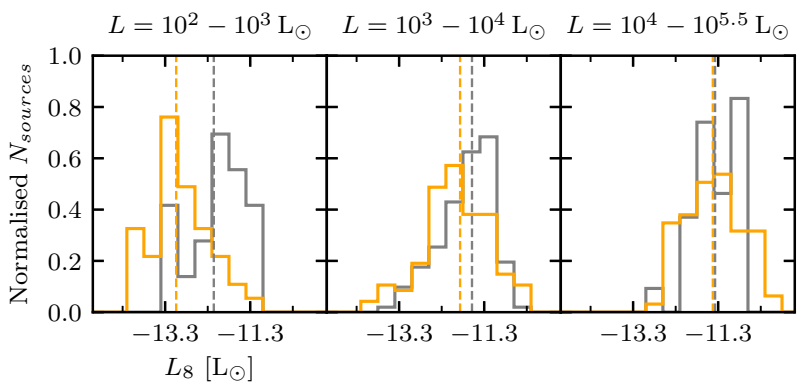

Figure 17. The distribution of $8 \mu \mathrm{m}$ spectral luminosities for maser (orange) and non-maser (grey) sources with a counterpart in the GLIMPSE point source catalogues, binned by FIR luminosity (increases left to right). The number of sources in each distribution and the values of the marked medians are given in Table 6.

sources found with $8 \mu \mathrm{m}$ GLIMPSE point sources counterparts. The distributions of $8 \mu \mathrm{m}$ luminosities for the maser and non-maser objects are shown in Figure 17 and summarised in Table 6 . On average the $8 \mu \mathrm{m}$ luminosities of the non-maser sources are found to be greater than the maser sources, a result evident as a significant difference through the use of KS tests. There is a more noticeable difference between the categories in the [8-70] $\mu \mathrm{m}$ colour, shown in Figure 18.

Finding the non-maser sources to have brighter $8 \mu \mathrm{m}$ counterparts is unlikely if the underlying evolutionary scenario is that these sources are less evolved. This therefore supports the suggestion that the matched non-maser sources are in a more evolved state. However, it should be noted that a significant number of objects in each sample are not associated with a compact $8 \mu \mathrm{m}$ counterpart.

As mentioned previously, there is a large overlap in all properties for the two types of clump and they are therefore not distinct types objects as seen in the infrared. A detailed investigation of all differences responsible for this behaviour

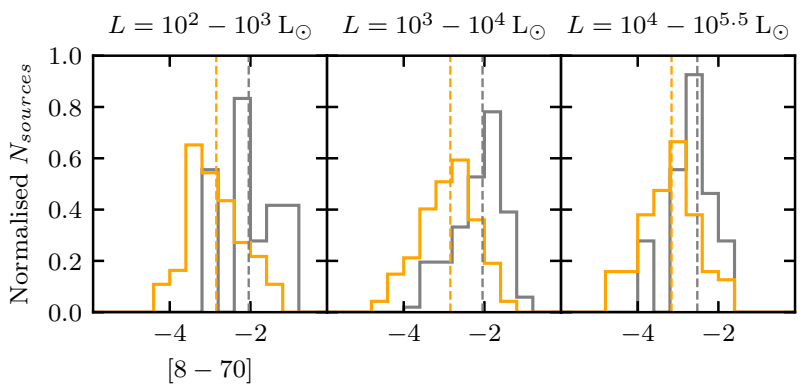

Figure 18. The distributions of [8-70] $\mu \mathrm{m}$ colour for maser (orange) and non-maser (grey) sources binned by FIR luminosity with a counterpart in the GLIMPSE point source. The distribution medians are given in Table 6.

requires follow-up observations. Observations to determine the chemistry of the two types of region would be beneficial, and detection of class II masers that are too weak to detect with the MMB or angled away from the line of sight would also result in re-categorisation of some non-maser objects.

To assess the plausibility of this evolutionary scenario, we consider the relative lifetimes of the two scenarios implied by the number of sources in each, taking the maser sources to occupy the mid evolutionary state in Figure 16 and the matched protostellar sources to occupy the final scenario. A comparison of the total number of sources in each category requires quantification of the sources missing from the total count for both the maser-bearing sources and the catalogue of Elia et al. (2017). In Section 5.6 we determined that the MMB is complete in methanol masers hosted by clumps of $>100 \mathrm{~L}_{\odot}$ within $2.7 \mathrm{kpc}$, and that we do not miss a significant number of nearby methanol maser sources due to sensitivity. However, the number of clumps hosting methanol maser sources requires correction for by the percentage not detected over multiple wavelengths in Hi-GAL as well as the percentage of the sample that lacks a reliable distance value. As we do not detect $33 \%$ of maser host clumps over all re- 
Table 6. Median values for the mid-infrared properties derived from $8 \mu \mathrm{m}$ data for the maser and non-maser objects with a counterpart in the GLIMPSE catalogues to investigate the likelihood of each scenario shown in Figure 16. The sources are binned in luminosity, with the sample median in each bin given for the two types of object, with the KS p-value reported in the bottom row. The corresponding distributions are shown in Figure 17 and 18.

\begin{tabular}{llllllllll}
\hline & \multicolumn{3}{c}{$\operatorname{Bin} 1\left(10^{2}-10^{3} \mathrm{~L}_{\odot}\right)$} & \multicolumn{3}{c}{ Bin $2\left(10^{3}-10^{4} \mathrm{~L}_{\odot}\right)$} & \multicolumn{3}{c}{ Bin $3\left(10^{4}-10^{5.5} \mathrm{~L}_{\odot}\right)$} \\
& No. & $L_{8}\left[10^{-12} \mathrm{~L}_{\odot}\right]$ & {$[8-70] \mu \mathrm{m}$} & No. & $L_{8}\left[10^{-12} \mathrm{~L}_{\odot}\right]$ & {$[8-70] \mu \mathrm{m}$} & No. & $L_{8}\left[10^{-12} \mathrm{~L}_{\odot}\right]$ & {$[8-70] \mu \mathrm{m}$} \\
\hline Non-maser & 18 & 0.70 & -2.04 & 128 & 2.59 & -2.05 & 27 & 4.24 & -2.52 \\
Maser & 46 & 0.09 & -2.85 & 118 & 1.34 & -2.84 & 79 & 3.75 & -3.16 \\
KS p-value & - & $10^{-8}$ & $10^{-8}$ & - & $10^{-9}$ & $10^{-14}$ & - & $10^{-11}$ & $10^{-11}$ \\
\hline
\end{tabular}

quired Hi-GAL bands and lack a distance for $18 \%$ of the sources in the sample, the total correction factor to obtain the true number of methanol maser hosts is 1.8. A factor of 4 correction is required for the protostars to account for those objects without reliable distance determinations.

Within $2.7 \mathrm{kpc}$ we find 50 maser sources and 9 matched non-maser sources, corrected to 90 and 36 sources respectively following the above. This implies the lifetimes to be within a factor of approximately 2.5, which we take to be reasonable for the proposed evolutionary scenario.

In the case that a significant population of $6.7 \mathrm{GHz}$ masers have a preferred beaming direction (such as a circumstellar disks with a restricted beaming angle centred on the disk planes), we would expect a population of matched maserless sources identical to the host clumps. However, the sources without a detected maser are found to be weaker at $70 \mu \mathrm{m}$, less likely to be associated with a point-like $8 \mu \mathrm{m}$ counterpart and more luminous at $8 \mu \mathrm{m}$, and so are not an identical population to the maser host clumps. This result suggests that it is unlikely that a significant fraction of the Galactic class II methanol maser population have a preferred beaming direction, consistent with recent VLBI observations (Sec. 5.6).

\section{MID-INFRARED RESULTS}

For the 292 maser host clumps with a counterpart identified at both $8 \mu \mathrm{m}$ and $22 / 24 \mu \mathrm{m}$, the colour-colour diagram between the [8-24] $\mu \mathrm{m}$ and [70-160] $\mu \mathrm{m}$ colour is shown in Figure 19, displaying no evidence of correlation. For the maser sources with $8 \mu \mathrm{m}$ and $24 \mu \mathrm{m}$ counterparts identified, the $[70-160] \mu \mathrm{m}$ colour is narrowly distributed with a $1-\sigma$ of 0.20 about a median of -0.10 . The [8-24] $\mu \mathrm{m}$ colour shows a larger scatter, with a median of -1.48 and standard deviation of 0.55 . A similar result is obtained for the [8-24] $\mu \mathrm{m}$ and [2470] $\mu \mathrm{m}$ colour-colour diagram shown in Figure 20, where the [24-70] $\mu \mathrm{m}$ colour has a median of -1.51 and standard deviation of 0.43 . A likely cause of the increasing scatter at 8 and $24 \mu \mathrm{m}$ is the dependence of flux on the viewing angle of the clump as the increase in opacity towards shorter wavelengths causes a high dependence on the amount of obscuring envelope material along the line of sight. For example, a line of sight cleared of dust through outflow activity results in a greater flux than if the same object was observed perpendicular to the outflow axis. Often observed to be associated with water maser emission, an outflow system such as this is a likely scenario for the clumps hosting methanol masers.

The final mid-infrared data associated with the methanol masers in this work is the catalogue of EGOs

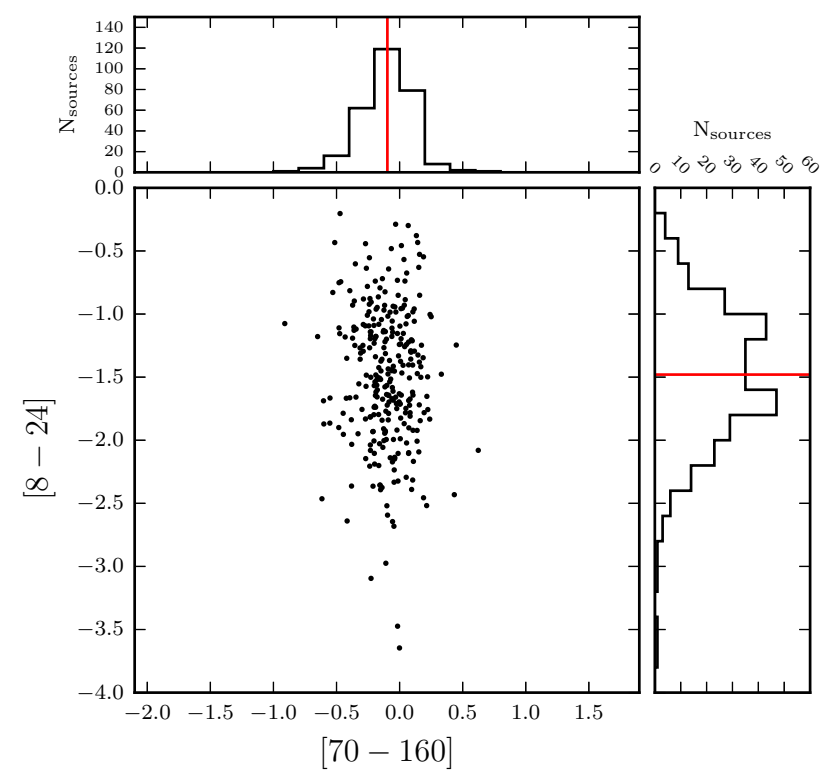

Figure 19. Colour-colour diagram of mid-infrared [8-24] $\mu \mathrm{m}$ colour against $[70-160] \mu \mathrm{m}$ colour shown in Section 5.5 with the axis scale set to be equal. The respective marginal distributions are shown for each property, with the medians marked in red at -1.48 and -0.1 for [8-24] $\mu \mathrm{m}$ and [70-160] $\mu \mathrm{m}$ colour respectively, with corresponding $1-\sigma$ widths of 0.55 and 0.20 .

produced by Cyganowski et al. (2008), with counterparts again identified through positional association with a maximum separation of 5 arcsec. The survey limits of GLIMPSE, from which this catalogue is derived, contain 528 of the 647 methanol masers in the sample. Of these, 86 are found to display EGO emission, yielding an association percentage of 16.3 per cent. The $6.7 \mathrm{GHz}$ masers with an associated EGO are found to be indistinct in every derived property of the host clump aside from the mass surface density. As shown in Figure 21, the clumps with an EGO are found at a median mass surface density of $1.61 \mathrm{~g} \mathrm{~cm}^{-2}$, which is significantly greater than that of those without an EGO (KS p-value $\sim 10^{-9}$ ) with a median value of $0.60 \mathrm{~g} \mathrm{~cm}^{-2}$. 


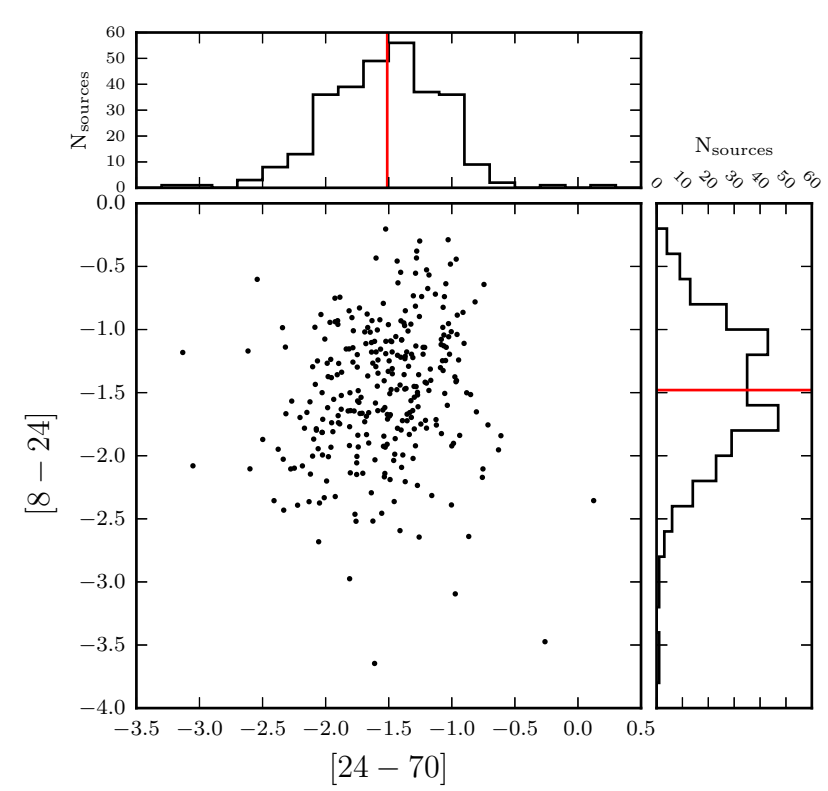

Figure 20. The [8-24] $\mu \mathrm{m}$ colour plotted against [24-70] $\mu \mathrm{m}$ colour with the axis scale set to be equal. As shown in the marginal distribution on the y-axis, the [8-24] $\mu \mathrm{m}$ colour has a median of -1.48 (red) and standard deviation of 0.55. Similarly, the $[24-70] /$ microns colour has a median of -1.51 and a standard deviation of 0.43 .

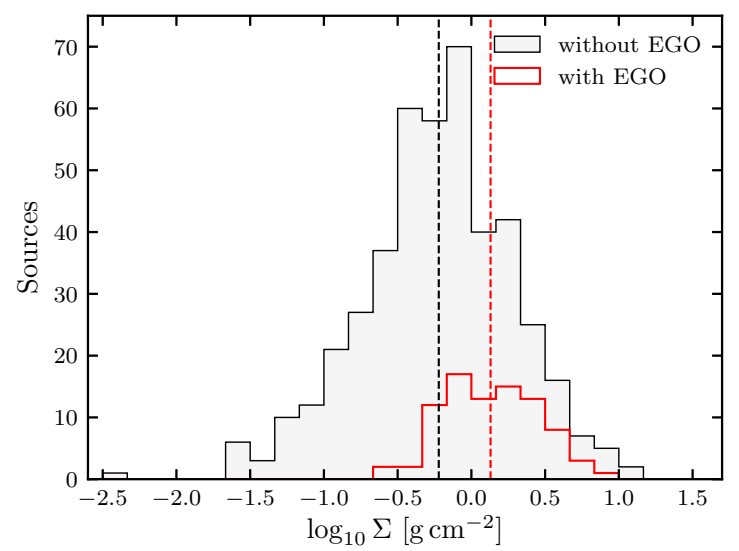

Figure 21. Distribution of surface densities for maser host clumps with (red) and without (grey) an associated EGO, with respective medians of $1.61 \mathrm{~g} \mathrm{~cm}^{-2}$ and $0.60 \mathrm{~g} \mathrm{~cm}^{-2}$ marked by the vertical lines.

\section{EVOLUTIONARY DIFFERENCES IN MASER ASSOCIATIONS}

\subsection{Identification of counterpart masers}

All maser catalogues detailed in Section 2.2 were used to find other maser emission arising from similar on-sky coordinates, and therefore the same protostellar host clump, for each $6.7 \mathrm{GHz}$ maser in the sample selected for this work. The survey range for both the $12.2 \mathrm{GHz}$ and excited hydroxyl masers matches that of the MMB survey as the $12.2 \mathrm{GHz}$ study was a targeted follow-up to all MMB sources by Breen et al. (2012a,b, 2014, 2016) and the ex-OH masers reported by Avison et al. (2016) which were co-observed during the MMB survey. An ex-OH maser is taken to be associated with a $6.7 \mathrm{GHz}$ source if the on-sky separation is less than $2.0^{\prime \prime}$. Breen et al. (2018) also identified associations between the 6.7 GHz MMB masers and the water masers from the HOPS data, with positional association a more complex task due to the less localised nature of water maser emission. The authors take this into account, and $6.7 \mathrm{GHz}$ methanol maser is associated with a water maser if a HOPS maser spot falls within 2.0 arcsec.

A full analysis of maser associations and their number statistics over the full Galactic Plane is presented in Breen et al. (2018). For this work, we are only concerned with investigating population differences in FIR-derived properties of protostellar clumps hosting $6.7 \mathrm{GHz}$ class II methanol masers based on the presence of additional maser emission, i.e. to allow determination of whether certain masers define a particular evolutionary stage or subset of class II methanol maser hosts. Within our sample of $647 \mathrm{Hi}-\mathrm{GAL}$ bright masers, 291 are listed to have observed $12.2 \mathrm{GHz}$ emission and from the sample of 127 ex-OH masers, 32 are associated with a clump in the Hi-GAL bright $6.7 \mathrm{GHz}$ host sample. Over a limited range of $20^{\circ}$ in Galactic longitude, 31 of 143 clumps are also associated with ground state hydroxyl maser sites detected with the SPLASH survey. From the 501 host clumps within the HOPS survey region, 122 are associated with water maser emission. The percentage of methanol masers also associated with a water maser improves with the addition of the more sensitive Titmarsh et al. (2014) and Titmarsh et al. (2016) studies towards a subset of 217 of these clumps, giving 110 associations.

\subsection{GHz class II methanol masers}

As for the $6.7 \mathrm{GHz}$ maser line, the $12.2 \mathrm{GHz}$ class II methanol masers are radiatively pumped by the re-emission of the strong UV radiation from the embedded protostar as infrared by nearby dust, and therefore are also co-located with the central protostellar object in a MYSO. This is the most common additional maser line observed towards the $6.7 \mathrm{GHz}$ host clumps, with approximately half (291 of 647) of the sample exhibiting both lines. We find no difference between the $12.2 \mathrm{GHz}$ maser properties in the sample in this paper and the complete sample of $43112.2 \mathrm{GHz}$ masers detected in the follow-up study (Breen et al. 2012a,b, 2014, 2016, Section 2.2). Similarly, the percentage of $6.7 \mathrm{GHz}$ masers with an additional $12.2 \mathrm{GHz}$ detection is not found to be a function of distance and is approximately constant at 45 per cent. Both of the above indicate that we have not biased the sample of $6.7 \mathrm{GHz}$ masers also with $12.2 \mathrm{GHz}$ detections by selecting only those with visible Hi-GAL counterparts, and our sample is representative of the entire population.

Figure 22 shows the distributions of the FIR-derived properties for the host clumps with and without $12.2 \mathrm{GHz}$ maser emission detected. The medians and KS p-values shown in the top row of Table 7 are used to determine which properties display a significant difference based on this categorisation. A significant difference is found in the mass surface density (Figure 22, second panel from left). Clumps emitting at $12.2 \mathrm{GHz}$ have at a median surface density of $0.94 \mathrm{~g} \mathrm{~cm}^{-2}$ whereas the clumps with no $12.2 \mathrm{GHz}$ emission 
Table 7. The median properties of $6.7 \mathrm{GHz}$ host clumps associated with a secondary maser of a given type, and for clumps lacking the same maser. The KS p-values are also given to assess whether the presence (or lack) of the maser holds any significance for each clump property.

\begin{tabular}{llcccccc}
\hline Maser & & Number & $T[\mathrm{~K}]$ & $\Sigma\left[\mathrm{g} \mathrm{cm}^{-2}\right]$ & $M\left[\mathrm{M}_{\odot}\right]$ & $L_{\mathrm{FIR}}\left[\mathrm{L}_{\odot}\right]$ & $L / M\left[\mathrm{~L}_{\odot} / \mathrm{M}_{\odot}\right]$ \\
\hline \multirow{2}{*}{$12.2 \mathrm{GHz} \mathrm{CH}_{3} \mathrm{OH}$} & with & 291 & 20.2 & 0.94 & 1440 & 6410 & 3.95 \\
& without & 356 & 19.2 & 0.60 & 1270 & 4420 & 4.50 \\
& p-value & - & 0.072 & $\sim 10^{-5}$ & 0.12 & 0.004 & 0.15 \\
$\mathrm{OH}$ & with & 31 & 22.0 & 1.53 & 1806 & 14330 & 7.02 \\
& without & 112 & 18.3 & 0.87 & 1538 & 4315 & 2.60 \\
\multirow{2}{*}{ Ex-OH } & p-value & - & $\sim 10^{-4}$ & 0.003 & 0.14 & 0.025 & $\sim 10^{-4}$ \\
& with & 32 & 22.2 & 1.61 & 1420 & 9040 & 7.82 \\
& without & 615 & 19.4 & 0.74 & 1340 & 5220 & 4.04 \\
$\mathrm{HOPS} \mathrm{H} \mathrm{H}_{2} \mathrm{O}$ & p-value & - & 0.012 & $\sim 10^{-4}$ & 0.97 & 0.12 & 0.005 \\
& with & 122 & 21.1 & 1.36 & 1570 & 9100 & 5.69 \\
& without & 379 & 19.3 & 0.67 & 1290 & 4800 & 3.96 \\
T14/T16 $\mathrm{H}_{2} \mathrm{O}$ & p-value & - & 0.002 & $\sim 10^{-8}$ & 0.26 & 0.006 & 0.004 \\
& with & 110 & 20.2 & 0.95 & 1884 & 8854 & 4.72 \\
& without & 107 & 17.9 & 0.72 & 2133 & 7649 & 2.79 \\
& p-value & - & 0.009 & 0.059 & 0.94 & 0.16 & 0.005 \\
\hline
\end{tabular}

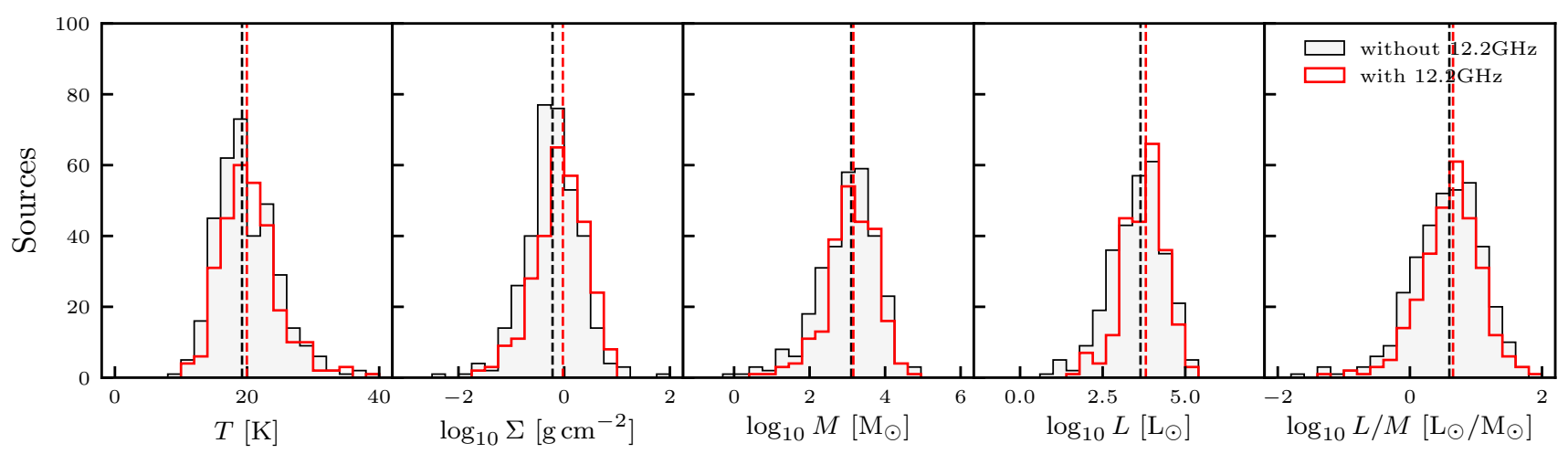

Figure 22. Distributions of the host clump properties derived in Section 5 categorised into clumps also associated with a $12.2 \mathrm{GHz}$ class II methanol maser detected (red) and those without (black). The median values of each distribution are given in Table 7 .

have a much lower sample median of $0.60 \mathrm{~g} \mathrm{~cm}^{-2}$, although this is still significantly higher than that of the general protostellar population. The median luminosities are 6410 and $4220 \mathrm{~L}_{\odot}$ respectively for clumps with and without $12.2 \mathrm{GHz}$ emission, and the host clumps with $12.2 \mathrm{GHz}$ are found to be more luminous (p-value of 0.004). Although there is a small increase in median temperature in sources which host a $12.2 \mathrm{GHz}$ maser, we do not find this to be statistically significant, similar to the result found by Breen et al. (2018) for similar samples.

\subsubsection{Hydroxyl masers}

Ground-state and excited-state $\mathrm{OH}$ maser lines are pumped by a combination of collisional and radiative processes (predominantly the latter, Field \& Gray 1988; Cragg et al. 2002). The ground state are found offset from the central protostar, typically associated with the edge of the enveloping ultracompact Hı region, whilst the excited-state are more closely bound to the protostar (e.g. Fish 2007; Caswell et al. 2009; Chanapote et al. 2019). Due to the association with the formation of an HiI region, we expect to find IR properties typical of the more advanced stages of protostellar evolution towards objects with a hydroxyl maser.

As described in Section 2.2, the excited state hydroxyl transition at $6035 \mathrm{MHz}$ was co-observed during the main MMB survey, therefore providing full coverage of the sample of Hi-GAL bright MMB masers for associations. The clumps associated with both a $6.7 \mathrm{GHz}$ methanol maser and an ex-OH maser are the smallest sample considered in this work with only 32 members, and statistically significant results are therefore more challenging to obtain. The distributions and median values of the IR properties are displayed in the bottom row of Figure 23 and Table 7 respectively for methanol masers associated with an ex-OH maser and those without. Despite the small sample size, we find statistically significant increases in T, $\Sigma$ and L/M for clumps also associated with an ex-OH maser, with large increases 
in median value for each. A median temperature of $22.1 \mathrm{~K}$ and mass surface density of $1.61 \mathrm{~g} \mathrm{~cm}^{-2}$ are consistent with the increase in temperature and surface density expected in the later stages of collapse, further supported by an increase in median $\mathrm{L} / \mathrm{M}$ to $7.82 \mathrm{~L}_{\odot} / \mathrm{M}_{\odot}$. There is no statistical evidence to suggest a difference in mass or luminosity between clumps with and without an ex-OH maser, and this IR analysis implies that the presence of an ex-OH maser is an evolutionary trait as opposed to occupying only the most luminous or most massive objects.

In addition to the ex-OH masers, the associations between SPLASH ground state $\mathrm{OH}$ maser sites and MMB masers performed by Qiao et al. (2016) and Qiao et al. (2018) are also used to separate the methanol maser sample into those with and without an association. Only the 143 clumps within the two SPLASH regions of $334^{\circ}<l<344^{\circ}$ and $-5^{\circ}<l<5^{\circ}$ through the Galactic centre are considered, giving a 31 masers associated with a ground-state $\mathrm{OH}$ transition and 112 without. As for the ex-OH masers, we find a comparable increase in median clump temperature, mass surface density and luminosity to mass ratio for $6.7 \mathrm{GHz}$ clumps associated with a ground state $\mathrm{OH}$ maser, with no distinction in clump mass. A significant increase in luminosity is also found towards methanol maser clumps with a secondary ground-state $\mathrm{OH}$ maser.

\subsubsection{Water masers}

The final maser association considered in this work is with water masers. These fundamentally differ from the previous maser types in that they are collisionally pumped, found in the regions associated with protostellar outflows and shocks in both high and low-mass star formation. This implies that the switch-on of secondary water maser emission in class II methanol maser sources may be at a slightly later evolutionary stage than the onset $6.7 \mathrm{GHz}$ maser emission as an outflow system must have sufficient time to develop from the protostellar feedback. However, the presence of a water maser can occur under other conditions aside from outflow activity in a massive star forming region, such as other shocks and PAH emission (Breen et al. 2014). To draw any conclusions about the switch-on of water masers in a $6.7 \mathrm{GHz}$ host clump, the assumption that they occupy a singular, albeit broad, stage of the evolutionary sequence of a massive star forming clump must be made. Breen et al. (2018) have shown that the inclusion of weak water masers in addition to those detected with HOPS would likely result in a Galactic water maser count in excess of the $6.7 \mathrm{GHz}$ population, even when accounting for those associated with low-mass star formation and evolved stars. This implies that this is a strong assumption, and any conclusions drawn about the placement of water masers in the evolutionary timeline for $6.7 \mathrm{GHz}$ sources is both only approximate and strictly only valid for sources also exhibiting luminous class II methanol maser emission.

Section 2.2 details the two studies considered in this work for water maser associations. The HOPS data provides the largest Galactic plane coverage for water maser detections, with 122 of the 501 maser host clumps within the survey range associated with a HOPS maser. However, the detection of water masers with HOPS is limited by the survey sensitivity of $\sim 5-10 \mathrm{Jy}$. The targeted follow-up study of water masers towards MMB sources presented in Titmarsh et al. (2014) and Titmarsh et al. (2016) reaches a sensitivity of $\sim 1.6 \mathrm{Jy}$ between Galactic longitudes $341^{\circ}$ and $20^{\circ}$ through the Galactic centre. For the 217 Hi-GAL bright methanol maser clumps in this restricted longitude region, the Titmarsh et al. studies recover all 54 HOPS masers associated with an FIR bright maser clump in our sample and provides an additional 56 associations (110 total). The distribution of peak flux for secondary masers assigned to the MMB host clumps for the two surveys is shown in Figure 24, showing the extra water masers that Titmarsh et al. (2014, 2016) find at low flux densities relative to HOPS when comparing the two regions that have been observed with both surveys. The distribution of peak flux densities of all HOPS masers found to be associated with a Hi-GAL bright $6.7 \mathrm{GHz}$ clump is also shown in shaded grey. Whilst the HOPS sample is likely to return more statistically significant results by providing broader coverage, we make use of the second sample to confirm whether observed differences in the properties of HOPS associated clumps are likely caused by the sensitivity of HOPS.

Restricted to the range of the HOPS survey to ensure uniform coverage of the sources for association, the sources with a water maser detected in HOPS are found to be hotter, at higher mass surface density, more luminous and at greater $L / M$ on average with statistical significance, although the median values shown in Table 7 are still somewhat lower than those obtained for the ex-OH clumps. The distributions of clump properties for sources with and without a secondary HOPS maser are shown in Figure 25.

Performing the same analysis with this restricted longitude range as for the HOPS associations, the sample medians and distributions for clumps associated with a Titmarsh et al. water maser are also shown in Table 7 and Figure 25. Whilst the statistically significant average increase in temperature and $L / M$ towards the clumps with a secondary water maser is also found for this sample, the clear statistical distinction in mass surface density and luminosity is not. This is plausibly due to the sensitivity of HOPS, as the FIR clump luminosity is found to be correlated with the $6.7 \mathrm{GHz}$ maser luminosity in Section 8.2, and the luminosity of a secondary water maser has in turn found to be correlated with the $6.7 \mathrm{GHz}$ methanol maser (Titmarsh et al. 2014, 2016). It is often similarly assumed that the water masers hosted in clumps with higher mass surface density are likely to be more luminous and so above the HOPS sensitivity threshold as higher densities enhance the collisional pumping of the maser line, although it should be noted that Elitzur et al. (1989) find that this is not consistent with their model.

\subsection{Relationship between infrared and maser properties}

The FIR properties of the host clumps may also be compared to the properties of a host maser itself. For each $6.7 \mathrm{GHz}$ maser, the MMB catalogue entry contains the peak flux, the flux integrated over the maser line and the maximum and minimum velocity obtained from the spectrum, giving an observed velocity range. Each of the above maser properties was checked for correlation with the FIR properties obtained, with maser luminosity $\mathrm{L}_{6.7}$ derived from the integrated flux of the line where a comparison of intrinsic quan- 


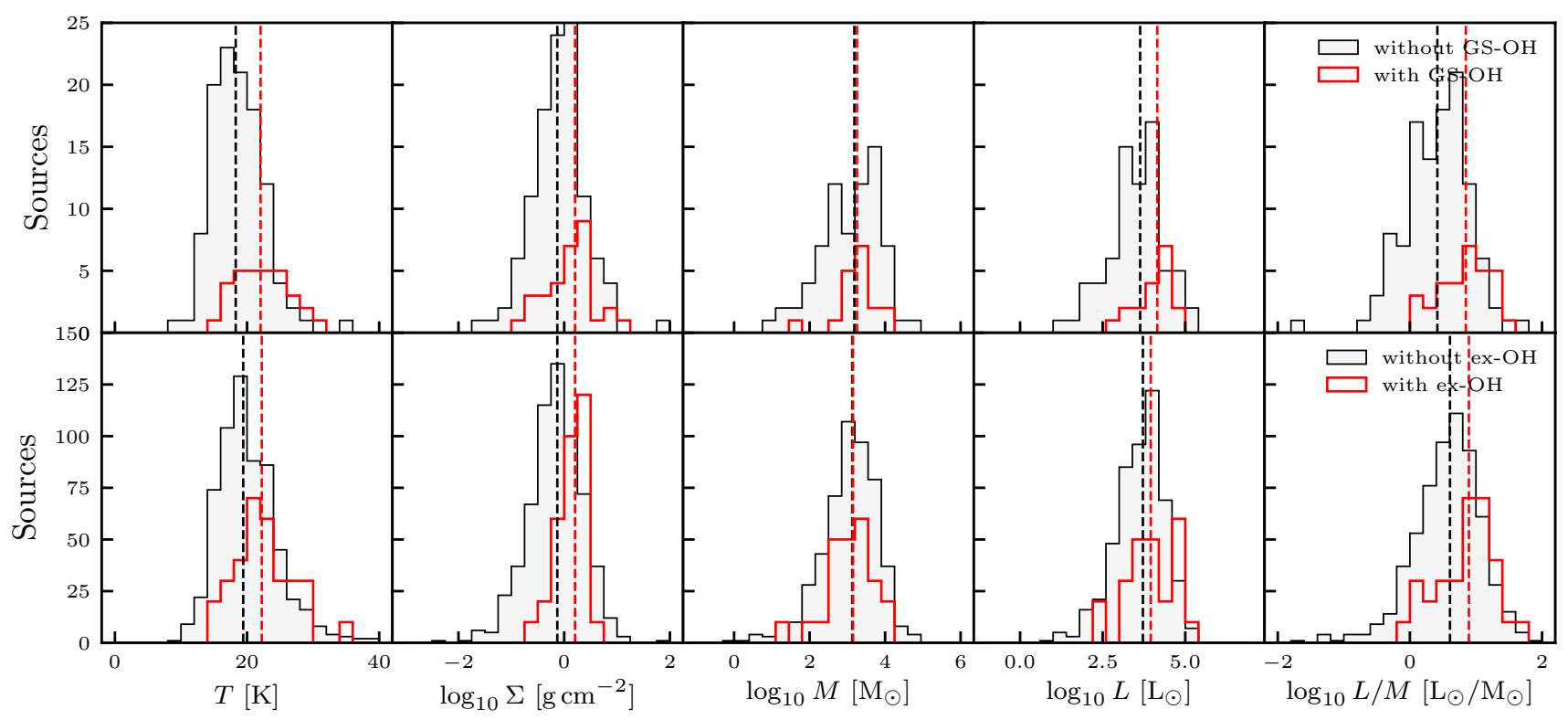

Figure 23. For clumps with (red) and without (black) a secondary hydroxyl maser detection, the distributions of host clump properties are shown with the marked median values given in Table 7 . The top row shows the distribution of secondary ground state hydroxyl masers detected for clumps within the SPLASH survey region. The bottom row displays the distribution of properties for clumps with excited state hydroxyl masers, covering all MMB sources. The number of masers in each bin with an ex-OH maser has been scaled up by a factor of 10 for visibility.

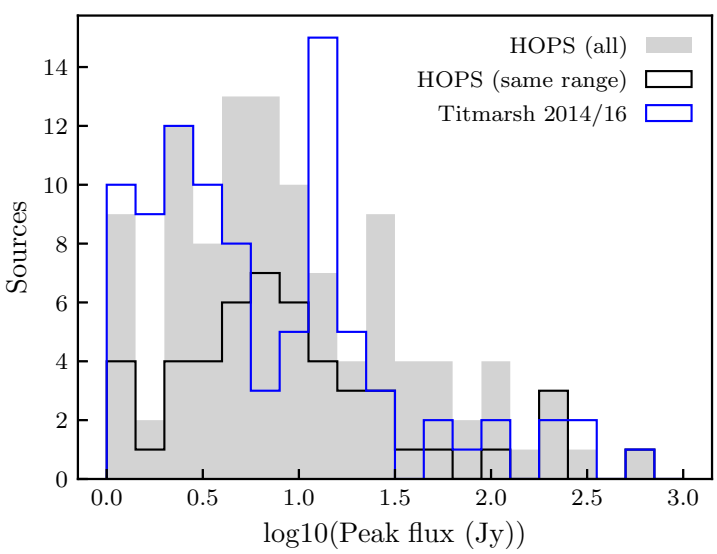

Figure 24. The peak flux density distributions for HOPS and the Titmarsh et al. samples of Galactic water masers that are associated with an MMB maser, showing a significant increase in the number of weak water masers recovered by the Titmarsh et al. samples (blue) relative to the HOPS detections in the same Galactic longitude range (black). The distribution of fluxes for the HOPS masers over the much larger entire survey range is also shown (grey shaded).

tities is required. We note here that we follow the convention of Green et al. (2017) and include a factor of $4 \pi$ when calculating maser luminosity.

No clear correlation is found for any maser property with $T, L_{\mathrm{FIR}}, M, \Sigma, L / M$ or any far-infrared or mid-infrared colour shown in Sections 5.5 and $7 . \mathrm{L}_{6.7}$ is shown against host clump $\mathrm{L}_{70}$ in Figure 26 and a general trend of increas- ing maser luminosity with $70 \mu \mathrm{m}$ luminosity is found for the sample. When logarithmically binned in $L_{6.7}$, the line of best fit through the median $L_{70}$ values returns a relationship of $\log _{10}\left(L_{70}\right)=(0.46 \pm 0.04) \log _{10}\left(L_{6.7}\right)+(3.1 \pm 0.1)$, with $70 \mu \mathrm{m}$ luminosity given in $\mathrm{L}_{\odot}$ and $6.7 \mathrm{GHz}$ maser luminosity in units of $\mathrm{Jy} \mathrm{km} \mathrm{s}^{-1} \mathrm{kpc}^{2}$. Perhaps more clearly than the overall trend is the appearance of an apparent minimum $70 \mu \mathrm{m}$ flux required to sustain a maser of a given luminosity. An approximation of this limit is found from taking the line of best fit through the $5^{\text {th }}$ percentile in each bin, returning a steeper line of $\log _{10}\left(L_{70}\right)=(0.59 \pm 0.03) \log _{10}\left(L_{6.7}\right)+(1.6 \pm 0.1)$ in the same units. As both properties are dependent on the square of the source distance, the slope in logarithmic space would be 1 if this minimum was simply an effect of the flux sensitivity limit of both surveys. Given that the slope is significantly less than 1 , we conclude that this effect is physical in origin.

Similarly, for the secondary masers present in a $6.7 \mathrm{GHz}$ host clump, a comparison of IR clump luminosity with maser luminosity can be made. In addition to the $6.7 \mathrm{GHz}$ masers, integrated maser luminosities have also been calculated for the $12.2 \mathrm{GHz}$ class II methanol masers, $\mathrm{H}_{2} \mathrm{O}$ masers from Titmarsh et al. and the SPLASH OH masers. As several $\mathrm{OH}$ masers detected in the SPLASH survey may be associated with a single MMB $6.7 \mathrm{GHz}$ maser, the integrated $\mathrm{OH}$ luminosity of a clump is taken to be the sum of the integrated luminosities over all of its individual $\mathrm{OH}$ masers. This shows correlation between the clump and maser luminosities in each case, but also that the scatter about the average trend is significant. We take the average absolute offset from the line of best fit in both maser luminosity (ydirection) and clump luminosity (x-direction) to quantify 


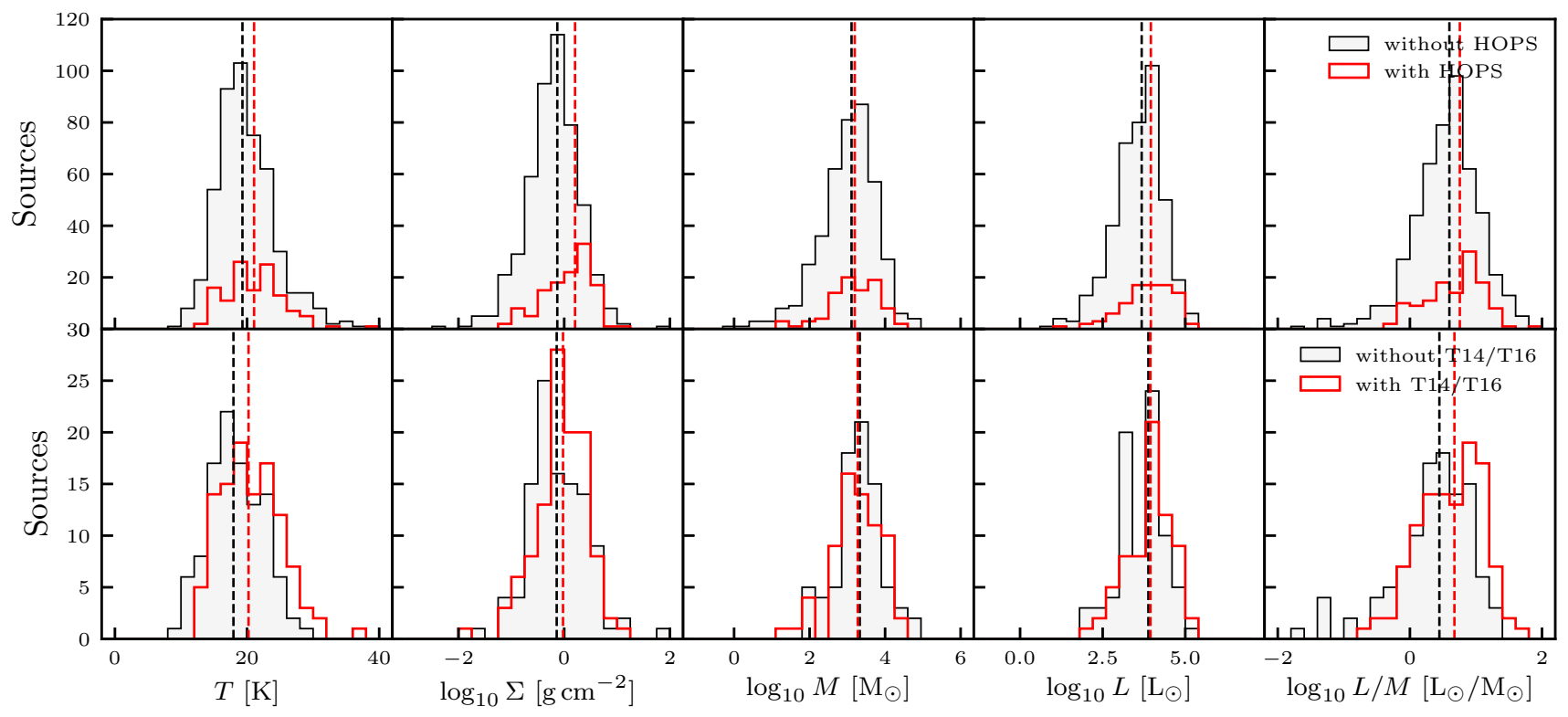

Figure 25. The distributions of properties for methanol maser clumps with (red) and without (black) a water maser detected in top: the HOPS survey and bottom: the Titmarsh et al. MMB follow-up studies. The median values for each distribution are given in Table 7 .

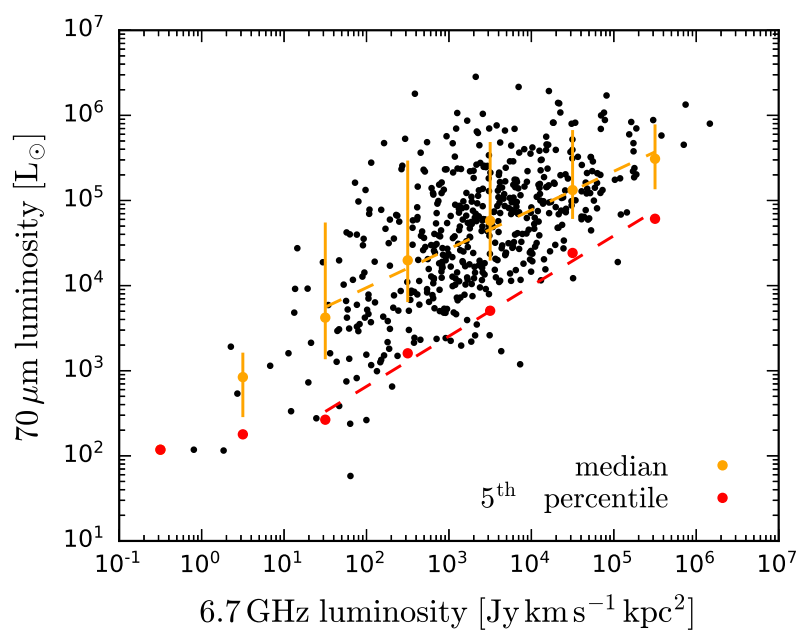

Figure 26. For maser host sources with a distance value available in the MMB catalogue, the $6.7 \dot{\mathrm{GH}} \mathrm{z}$ methanol maser luminosity (integrated over the maser line) is shown against the $70 \mu \mathrm{m}$ clump luminosity for each source. Binning in maser luminosity, the general trend between the two properties is characterised by the line through the median $70 \mu \mathrm{m}$ luminosity (yellow) in each bin, given by $\log _{10}\left(L_{70}\right)=(0.46 \pm 0.04) \log _{10}\left(L_{6.7}\right)+(3.1 \pm 0.1)$ and shown with the standard deviation in each bin. Similarly, an approximate lower threshold on the required $70 \mu \mathrm{m}$ flux to sustain a class II methanol maser of a given luminosity is given by the $5^{\text {th }}$ percentile in each bin (red), with a the line of best fit given by $\log _{10}\left(L_{70}\right)=(0.59 \pm 0.03) \log _{10}\left(L_{6.7}\right)+(1.6 \pm 0.1)$. The $70 \mu \mathrm{m}$ luminosity and maser luminosity are given in units of $\mathrm{L}_{\odot}$ and $\mathrm{Jy} \mathrm{km} \mathrm{s}{ }^{-1} \mathrm{kpc}^{2}$.
Table 8. The lines of best fit and average deviation from this line in each direction for maser luminosity against host clump FIR luminosity. From left to right, the columns are: maser type, number used to calculate the line, the line of best fit parameters of the form $\log _{10}\left(L_{\mathrm{m}}\right)=\mathrm{p}_{1} \log _{10}\left(L_{\mathrm{FIR}}\right)+\mathrm{p}_{2}$, and the average absolute logarithmic offset from this line in clump luminosity and maser luminosity ( $\mathrm{x}$ - and $\mathrm{y}$-direction respectively in Figure B1).

\begin{tabular}{lccccc}
\hline Maser & $\mathrm{N}$ & \multicolumn{2}{c}{ Fit parameters } & \multicolumn{2}{c}{ Average offset } \\
& & $\mathrm{p}_{1}$ & $\mathrm{p}_{2}$ & $\log _{10}\left(L_{\mathrm{FIR}}\right)$ & $\log _{10}\left(L_{\mathrm{m}}\right)$ \\
\hline $6.7 \mathrm{GHz}$ & 511 & $0.8 \pm 0.1$ & $0.4 \pm 0.2$ & 0.7 & 0.6 \\
$12.2 \mathrm{GHz}$ & 219 & $0.7 \pm 0.1$ & $0.0 \pm 0.3$ & 0.9 & 0.6 \\
$\mathrm{H}_{2} \mathrm{O}$ & 67 & $0.7 \pm 0.1$ & $1.5 \pm 0.5$ & 0.7 & 0.5 \\
$\mathrm{OH}$ & 18 & $0.9 \pm 0.2$ & $-0.5 \pm 0.9$ & 0.4 & 0.4 \\
\hline
\end{tabular}

this scatter, with each of these given alongside the line of best fit parameters in Table 8. In each case, approximately half an order of magnitude of scatter in maser luminosity is found for a given clump luminosity, and vice versa. This result is unsurprising given that maser luminosity depends on stochastic properties, such as coherence and beaming, and is consistent with the long-term variability found by Ellingsen (2007). This therefore implies that although a correlation is evident, the use of one given luminosity to predict another in a host clump from the line of best fit relationship is only accurate to an order of magnitude at best, and that a 1:1 correlation of the two properties is not representative of the data.

The correlation between the luminosity of the $6.7 \mathrm{GHz}$ maser and secondary masing species in a host MYSO has also been studied for the targeted MMB follow-ups, so as in Breen et al. (2016) and Titmarsh et al. (2014, 2016) for the $12.2 \mathrm{GHz}$ and water masers respectively, we present this for the host clumps in this sample and the SPLASH 
$\mathrm{OH}$ masers. As for the correlations between maser and host clump luminosity, the line of best fit is given by $\log _{10}\left(L_{\mathrm{OH}}\right)=$ $(0.6 \pm 0.2) \log _{10}\left(L_{6.7}\right)+(0.5 \pm 0.7)$, with an average logarithmic scatter of 0.7 in $6.7 \mathrm{GHz}$ luminosity and 0.4 in total $\mathrm{OH}$ luminosity from this line.

The accompanying plots for these correlations are presented in Appendix B

\subsection{Comparison of all associations}

Comparison between the types of secondary maser associations allows the relative evolutionary stage of the protostellar host clump in each category to be assessed through its typical IR properties. In addition to the above associations, we consider two more categories: 'solitary' $6.7 \mathrm{GHz}$ methanol masers for which no additional masers have been identified, and 'associated' masers, in which the host clump has one or more additional masers of any type detected.

To ensure that all methanol maser host clumps are equally checked for secondary masers, the longitude range of the associated and solitary categories must be equal to the smallest survey range considered. Due to the small onsky coverage of the Titmarsh et al. and SPLASH surveys, the sample sizes that would remain by restricting to their common coverage would be challenging to obtain statistically significant results from. We therefore do not use these surveys when determining whether a $6.7 \mathrm{GHz}$ host clump also contains a secondary maser and falls within the 'associated' category. As shown in Section 8.1.3, the HOPS survey recovers the strongest water masers. The 'associated' category is therefore characterised by the presence of a second class II methanol maser line at $12.2 \mathrm{GHz}$, an excited-state hydroxyl maser or a strong water maser, and the 'solitary' category may have weak water masers or ground-state hydroxyl masers present. The remaining survey with the smallest on-sky coverage is HOPS, and the new number of sources and median properties for each category when restricting to this region are displayed in Table 9 .

Figure 27 shows all properties as box-and-whisker plots to identify trends between the categories. We note that the mass distributions of each category are statistically indistinct from one another, implying that the appearance of any given secondary maser is not mass dependent given that a host clump is sufficiently massive to host a class II methanol maser. In each of the other properties, one or more secondary masers of any type present in a clump traces sources of greater $T, \Sigma, L$ and $L / M$ on average, relative to solitary $6.7 \mathrm{GHz}$ masers. As expected from previously proposed evolutionary schemes such as that of Breen et al. (2010) and taking $T, L$ and $\Sigma$ to increase simultaneously with evolution, clumps with $12.2 \mathrm{GHz}$ emission are found to be at an earlier evolutionary stage than those with secondary excited hydroxyl masers. Similarly, these results alone imply that the onset of water masing occurs at a later time than the switchon of $12.2 \mathrm{GHz}$ emission. However, Section 8.1.3 shows that the detection limit of HOPS limits this conclusion to the onset of strong secondary water masing. The use of the more sensitive Titmarsh et al. samples removes the statistically significant difference in the distribution of $\Sigma$ for sources with and without a water maser. Although the difference in $T$ and $L / M$ remains, constraining the evolutionary state of clumps with 6.7 and $22 \mathrm{GHz}$ water masers will require a greater lon- gitude coverage with a sensitive survey. When considering these results, it should still be noted that the overlap in both proposed evolutionary stage and the IR properties of host clumps in each association category is considerable.

\subsection{Timeline of secondary masers}

As in Breen et al. (2010), the statistical lifetime of each secondary maser phase within the $6.7 \mathrm{GHz}$ lifetime can be estimated from the number of clumps associated with each and a timeline constructed. The lifetimes estimated here are only for clumps that simultaneously host a class II methanol maser alongside each type of secondary maser, and we do not sample other evolutionary states which may be associated with these masers outside of the $6.7 \mathrm{GHz}$ lifetime. We calculate the relative lifetime of each secondary maser species from the percentage of $6.7 \mathrm{GHz}$ masers and the results are given in Table 10. As each of the input surveys has different detection limits due to varying sensitivities, each of these relative lifetimes is a lower bound on the true value as undetected weak secondary masers may be present. An example of this is the difference in lifetime estimates from the HOPS survey of only bright water masers and the more sensitive survey by Titmarsh et al. (2014, 2016), with the detection of weak water masers significantly increasing the estimated lifetime. Converting the relative timescales requires an estimate of the lifetime $6.7 \mathrm{GHz}$ maser. van der Walt (2005) estimate the lifetime to be between $2.5 \times 10^{4}$ and $4.5 \times 10^{4} \mathrm{yr}$, with Billington et al. (2019) recently deriving a consistent lifetime of $3.3 \times 10^{4} \mathrm{yr}$ from analysis of data from the ATLASGAL survey.

An estimate of the relative location of each secondary maser within the $6.7 \mathrm{GHz}$ lifetime is given by the statistical overlap between each species. For example, $60 \%$ of the ex$\mathrm{OH}$ masers are found in clumps that also host a $12.2 \mathrm{GHz}$ maser, and this implies that the two lifetimes should overlap by $60 \%$ of the ex-OH lifetime given in Table 10. As this method does not identify whether a maser species should overlap with the start or end of the lifetime of another species, the order of appearance within this scheme is given by the sequence of increasing $L_{F I R} / M$ shown in Figure 27. The switch-on time of secondary masing is also not constrained by this method, and so the location of this sequence within the lifetime of a $6.7 \mathrm{GHz}$ maser is not fixed.

Considering the overlap between multiple different secondary species also yields different locations within the timeline for both the $\mathrm{OH}$ and ex-OH masers. The black lines in Figure 28 are the locations calculated from the overlap with $12.2 \mathrm{GHz}$ masers for each species, and the red and orange from the overlap with water and $\mathrm{OH}$ similarly.

It has been assumed that all maser types to form an evolutionary sequence and occupy single sub-phases of the lifetime of $6.7 \mathrm{GHz}$ masing. As we have already selected sources in a constrained evolutionary state through the presence of a $6.7 \mathrm{GHz}$ maser, it seems unlikely that there are several distinct sub-categories of object within this sample each with the conditions required to host each type of radiative secondary maser. However, this may be less true for the water masers as the collisional pumping is dependent on the interaction of a protostar with its environment (Breen et al. 2014). For this reason, water masers are much more difficult to reliably place on an evolutionary timeline using only sta- 
Table 9. Median properties of $6.7 \mathrm{GHz}$ maser host clumps categorised by secondary maser assessed within the same range of Galactic latitudes, including a 'solitary' category for which no secondary masers are found and an 'associated' category for which any additional maser is identified.

\begin{tabular}{lcccccc}
\hline Category & Number $(\%)$ & $<\mathrm{T}>[\mathrm{K}]$ & $\left\langle\Sigma>\left[\mathrm{g} \mathrm{cm}^{-2}\right]\right.$ & $<\mathrm{M}>\left[\mathrm{M}_{\odot}\right]$ & $<\mathrm{L}>\left[\mathrm{L}_{\odot}\right]$ & $<\mathrm{L} / \mathrm{M}>\left[\mathrm{L}_{\odot} / \mathrm{M}_{\odot}\right]$ \\
\hline All & $501(100)$ & 19.6 & 0.80 & 1470 & 5340 & 4.14 \\
Solitary & $204(41)$ & 19.0 & 0.55 & 1440 & 4190 & 3.61 \\
Associated & $297(59)$ & 20.0 & 0.97 & 1470 & 6630 & 4.73 \\
$12.2 \mathrm{GHz}$ & $232(46)$ & 20.0 & 0.96 & 1360 & 6380 & 4.53 \\
$\mathrm{H}_{2} \mathrm{O}$ & $122(24)$ & 21.0 & 1.36 & 1570 & 9100 & 5.61 \\
ex-OH & $29(6)$ & 21.7 & 1.53 & 1360 & 8970 & 7.73 \\
\hline
\end{tabular}

Table 10. Relative lifetimes of each secondary maser found in clumps hosting a $6.7 \mathrm{GHz}$ methanol maser. The percentage of clumps associated with each secondary maser have been calculated from the values in Table 7 .

\begin{tabular}{lcc}
\hline Species & Percentage & Lifetime (lower-upper) $\left[10^{4} \mathrm{yr}\right]$ \\
\hline $6.7 \mathrm{GHz}$ & 100 (reference) & $2.5-4.5$ \\
$12.2 \mathrm{GHz}$ & 45.0 & $1.1-2.0$ \\
$\mathrm{H}_{2} \mathrm{O}(\mathrm{HOPS})$ & 24.4 & $0.6-1.1$ \\
$\mathrm{H}_{2} \mathrm{O}(\mathrm{T} 14 / \mathrm{T} 16)$ & 50.7 & $1.3-2.3$ \\
$\mathrm{OH}$ & 21.7 & $0.5-1.0$ \\
$\mathrm{ex}-\mathrm{OH}$ & 4.9 & $0.1-0.2$ \\
\hline
\end{tabular}

tistical overlaps, and this is seen in incorporating the water masers detected in Titmarsh et al. into Figure 28. Similarly, the narrow lifetime and the uncertainty in positioning in the timeline of the ex-OH masers perhaps suggests that their presence is linked to very specific conditions, which may not be simply related to the evolutionary state of a protostar.

The small sample sizes of clumps with $\mathrm{OH}$ and ex-OH secondary masers contributes significant uncertainty to the statistical lifetime estimates, and is also a likely source of the uncertainty in their location within the evolutionary timeline when considering different overlaps. Additionally, the location changes when calculated from the overlap with the HOPS masers as these sample only bright water maser sources. It is also important to note that the lifetime of the ex-OH masers is a lower limit as several host clumps are saturated in the Hi-GAL maps and are therefore not included in our sample. When considering the entire sample of ex$\mathrm{OH}$ masers, Avison et al. (2016) estimate a lifetime between $3.3 \times 10^{3}$ and $8.3 \times 10^{3}$ yr while the statistical lifetime of $1 \times 10^{3}$ to $2 \times 10^{3} \mathrm{yr}$ is found in this work.

Determining the switch-on time of a secondary maser species within the $6.7 \mathrm{GHz}$ lifetime requires a robust evolutionary tracer that can be mapped to time. In principle the luminosity to mass ratio, for the appropriate circumstellar mass, could provide such a tracer, but its relationship to time is not well determined and may be a function of initial mass, and/or the ultimate stellar mass (and luminosity).

\section{SUMMARY}

We have characterised a complete sample of MYSOs hosting $6.7 \mathrm{GHz}$ methanol masers through combination of $\sim 1000$ of these masers detected with the Methanol MultiBeam survey and compact source catalogues for each of the Hi-GAL wavelengths over the full Galactic plane. We associate $96 \%$ of all masers with a compact source in at least one Hi-GAL wave- length. We find 73 per cent of all MMB class II methanol masers to be associated with a compact source in four HiGAL bands, yielding a sample of 647 maser host clumps for further analysis following removal of sources with unreliable flux estimates. For maser-bearing clumps of far-infrared luminosity $\geq 100 \mathrm{~L}_{\odot}$, we find the MMB to be complete to $2.7 \mathrm{kpc}$, taking both weak methanol masers and beaming into consideration.

Our key results are as follows:

i) We have derived the typical properties of the massive protostellar clumps hosting MMB masers by fitting to their far-infrared spectral energy distributions. The maser host clumps on the near and far side of the Galactic centre (taken to be at a heliocentric distance of $8.0 \mathrm{kpc}$ ) represent different populations due to the selection angular size used to create the Hi-GAL compact source catalogues. The typical mass, radius and far-infrared luminosity of nearby sources (median distance $3.8 \mathrm{kpc}$ ) are $630 \mathrm{M}_{\odot}, 0.2 \mathrm{pc}$ and $2500 \mathrm{~L}_{\odot}$, whereas the same properties for the maser hosts on the far side of the Galaxy (median distance $11.4 \mathrm{kpc}$ ) are $3200 \mathrm{M}_{\odot}, 0.6 \mathrm{pc}$ and $10000 \mathrm{~L}_{\odot}$ respectively. We do not detect small clumps on the far side of the Galaxy, confirmed by the lack of a clear multiwavelength detection of a host clump for all of the MMB sources. Despite the difference in typical size, we do not find these two populations to be distinct in luminosity-to-mass ratio, and thus are in the same relative evolutionary state. The median $L_{F I R} / M$ for maser host clumps is $4.2 \mathrm{~L}_{\odot} / \mathrm{M}_{\odot}$, and the median temperature and mass surface density are 19.5 and $0.76 \mathrm{~g} \mathrm{~cm}^{-2}$ respectively.

ii) We find that $6.7 \mathrm{GHz}$ methanol masers, and therefore highmass protostars, are present in clumps down to mass surface densities of approximately $0.08 \mathrm{~g} \mathrm{~cm}^{-2}$. This value is similar to thresholds of $\sim 0.1 \mathrm{~g} \mathrm{~cm}^{-2}$ for massive star formation reported by other work, despite derivation from significantly different methods.

iii) We have identified a correlation between the $6.7 \mathrm{GHz}$ inte- 

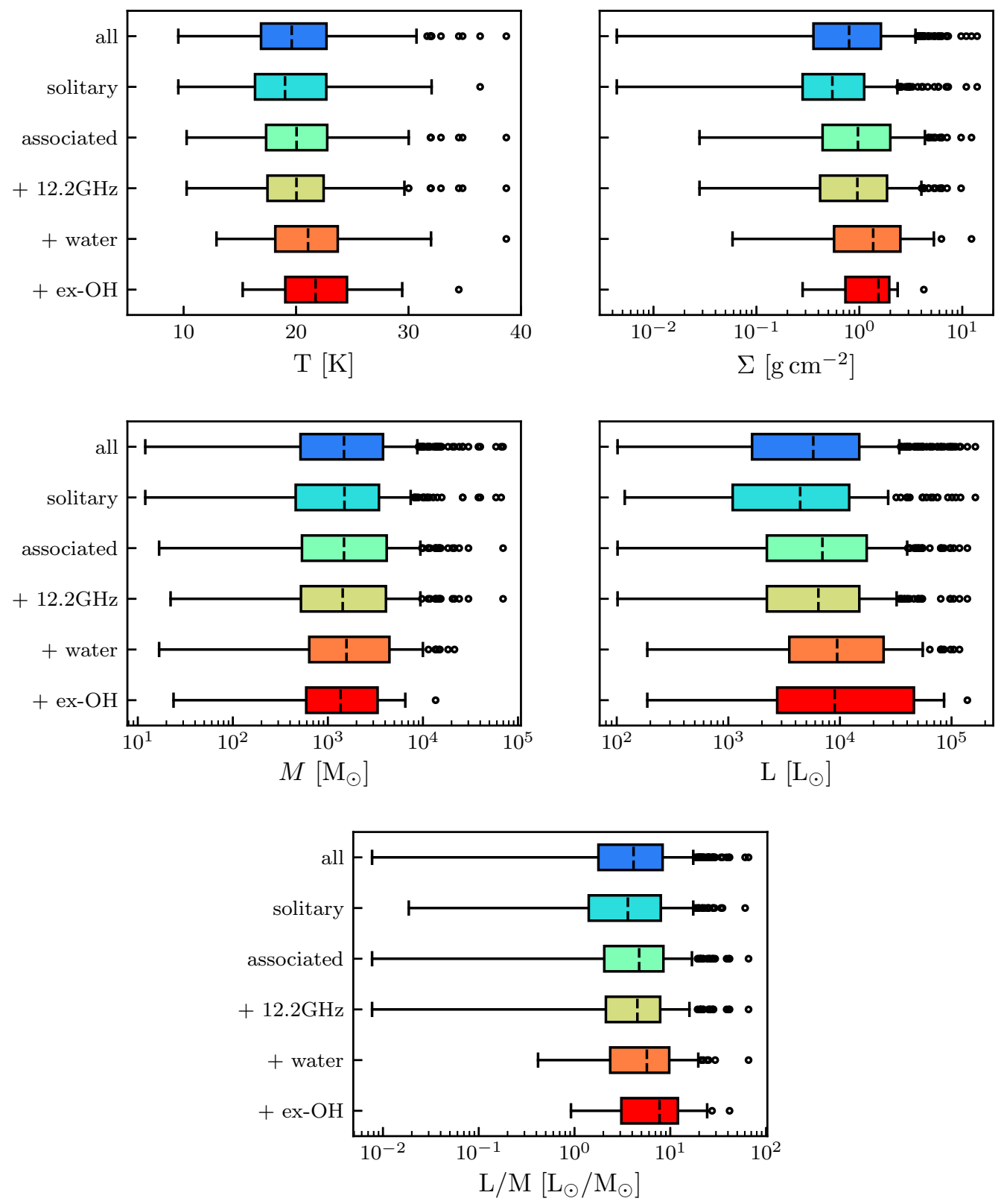

Figure 27. Box-and-whisker plots of the distribution of infrared-derived clump properties for objects hosting a $6.7 \mathrm{GHz}$ methanol maser, categorised by the presence of any given secondary maser. For each panel from top to bottom: the total maser sample, clumps hosting a methanol maser without any secondary masers found in the surveys used, clumps with at least one secondary maser of any type, and the distributions for clumps with each type of secondary maser $\left(12.2 \mathrm{GHz} \mathrm{CH}_{3} \mathrm{OH}, \mathrm{H}_{2} \mathrm{O}\right.$ from $\mathrm{HOPS}$, and excited-state hydroxyl). Each box is drawn between the lower (25th percentile) to upper (75th percentile) quartile value, with the length of the box defining the interquartile range (IQR) and a vertical dashed line at the median. The median values are given in Table 9. The whiskers (capped solid lines) give the range of the data, defined as $1.5 \times \mathrm{IQR}$ past each end of the box, and outliers beyond this are plotted as circles.

grated luminosity of a maser and the luminosity of its host clump at $70 \mu \mathrm{m}$, finding the minimum $70 \mu \mathrm{m}$ clump luminosity required to be associated with a maser of a given luminosity to be characterised by $L_{70} \propto L_{6.7}^{0.6}$.

iv) Class II methanol maser hosts are found to be at greater values in all clump properties relative to the population of all protostellar clumps visible with Hi-GAL. The range of $L_{F I R} / M$ covered by the maser sample is narrower relative to the spread in $L_{b o l} / M$ seen for the protostellar sample.
Identifying approximate thresholds on each property and through the use of PCA, we identify 896 protostellar objects, from a sample of 5497, that match the properties of the methanol maser host clumps but lack a detection of a strong $6.7 \mathrm{GHz}$ maser with the MMB survey. Accounting for completeness in both samples, we find a factor of 2.5 more maser sources than matched maserless clumps.

Finding a $70 \mu \mathrm{m}$ deficiency in the sources lacking a methanol maser, a comparison of the mid-infrared $8 \mu \mathrm{m}$ properties of 


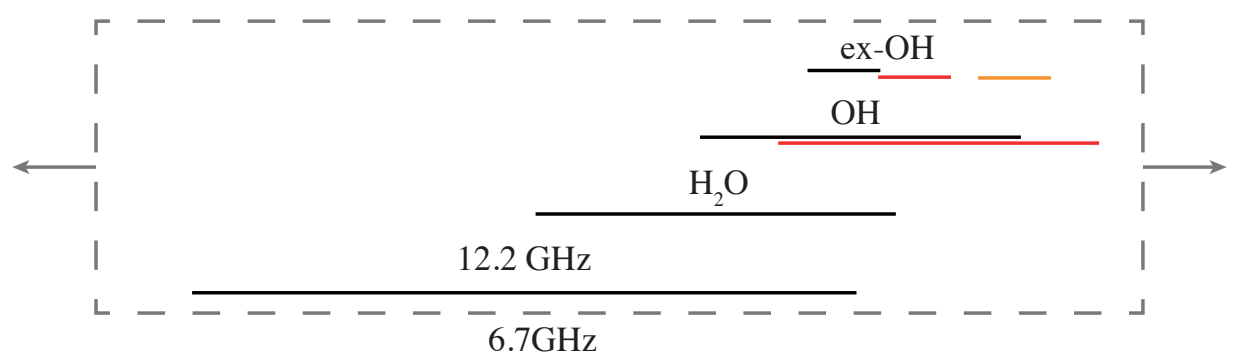

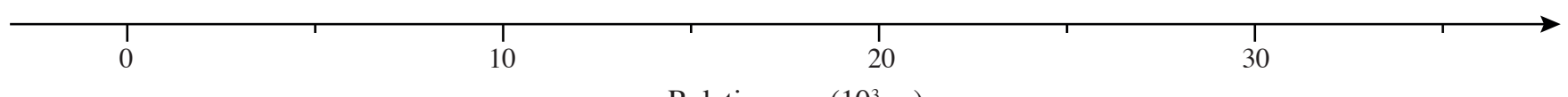

Relative age $\left(10^{3} \mathrm{yr}\right)$

Figure 28. Timeline of the relative duration and evolutionary offset of each secondary maser species found within $6.7 \mathrm{GHz}$ host clumps. The duration of the class II methanol maser phase is taken to be $3.5 \times 10^{4} \mathrm{yr}$ (van der Walt 2005) and length of the line is determined by the fraction of $6.7 \mathrm{GHz}$ methanol masers with this species. The offset between each species of secondary maser is given by the statistical overlap with the other secondary masers. The black, red and orange lines are found from considering the overlap with $12.2 \mathrm{GHz}$ class II methanol masers, water masers and ground-state hydroxyl masers respectively. The overall location of the secondary masers within a 6.7 $\mathrm{GHz}$ lifetime is not fixed, as indicated by the grey box.

these sources suggests that the objects lacking a $6.7 \mathrm{GHz}$ methanol maser may be in a later evolutionary stage. However, this conclusion is limited by the small number of sources in each sample with a detected GLIMPSE counterpart (19 and 47 per cent of sources in the non-maser and maser samples respectively). The origin of these low values needs further investigation. It is unlikely that the majority of these sources host weak methanol masers (§ 6.3). However, confirming this requires more sensitive observations while observations on smaller, core-scales will allow us to more fully understand the properties of the maser-less, embedded protostars and their apparent lack of methanol masers. This is the subject of future work.

v) Considering secondary water, hydroxyl and $12.2 \mathrm{GHz}$ class II methanol masers in each MMB maser host clump, we find that clumps associated with any secondary maser to be more evolved than those hosting a solitary $6.7 \mathrm{GHz}$ maser. Sources with a hydroxyl maser are found to be significantly more advanced in evolution. We find sources with water masers detected in HOPS to be at an intermediate evolutionary stage between the solitary $6.7 \mathrm{GHz}$ and hydroxyl-bearing clumps, although we do not find a statistically significant difference in luminosity or mass surface density relative to clumps associated with a water maser relative to those that are not when considering higher-sensitivity surveys. We find a $1 \mathrm{~K}$ median temperature increase of borderline statistical significance towards sources with a second class II methanol maser at $12.2 \mathrm{GHz}$. We present a maser timeline summarising these results.

vi) Although trends between FIR clump luminosity and maser luminosity are found for each type of secondary maser in a 6.7 GHz host clump, these relationships have order of magnitude scatter and are not 1:1 relationships. Any predictions of maser luminosity from infrared clump luminosities should account for this.

In summary, comparison of the sources identified in the Methanol MultiBeam (MMB) survey and the Herschel Infrared Galactic Plane Survey (Hi-GAL) has allowed the most comprehensive, Galaxy-wide, study of the properties and evolutionary status of the sources which host $6.7 \mathrm{GHz}$ methanol masers to date. The maser sources have median luminosity of $5600, \mathrm{~L}_{\odot}$ and are associated with clumps of median radius $0.42 \mathrm{pc}$ with median mass $1400 \mathrm{M}_{\odot}$, although these are influenced by the bi-modal distribution of distances to the sources. On the near side of the Galaxy the typical source has a luminosity of $2500, \mathrm{~L}_{\odot}$, lower than typically previously assumed for these sources. However, the clumps in which these sources are found have high surface densities, consistent with them eventually becoming high mass, and high luminosity, sources. Our analysis has identified for the first time a sample of sources which are similar to the maser sources in a range of properties but do not host masers. We show that these are consistent with being at a more evolved phase of evolution than the maser sources, but confirmation of this requires further comparative study of these objects.

\section{ACKNOWLEDGEMENTS}

BMJ acknowledges the support of a studentship grant from the UK Science and Technology Facilities Council (STFC). AA is funded by the STFC at the UK ALMA Regional Centre Node through grant ST/P000827/1. SPE acknowledges the support of an Australian Research Council Discovery Project (project number DP180101061). MM acknowledges support from the grant 2017/23708-0, São Paulo Research Foundation (FAPESP). This research has made use of the NASA/ IPAC Infrared Science Archive, which is operated by the Jet Propulsion Laboratory, California Institute of Technology, under contract with the National Aeronautics and Space Administration. We would like to thank Andrés Guzmán and Yanett Contreras for their very helpful discussion about the temperature of the maser sources. 


\section{REFERENCES}

Avison A., Peretto N., Fuller G. A., Duarte-Cabral A., Traficante A., Pineda J. E., 2015, Astronomy \& Astrophysics, 577, A30

Avison A., et al., 2016, Monthly Notices of the Royal Astronomical Society, 461, 136

Bartkiewicz A., Szymczak M., van Langevelde H. J., Richards A. M. S., Pihlström Y. M., 2009, Astronomy \& Astrophysics, 502,155

Bartkiewicz A., Szymczak M., van Langevelde H. J., 2014, Astronomy \& Astrophysics, 564, A110

Bartkiewicz A., Szymczak M., van Langevelde H. J., 2016, Astronomy \& Astrophysics, 587, A104

Benjamin R. A., et al., 2003, Publications of the Astronomical Society of the Pacific, 115, 953

Billington S. J., et al., 2019, preprint (arXiv:1907.00564)

Breen S. L., Ellingsen S. P., Caswell J. L., Lewis B. E., 2010, Monthly Notices of the Royal Astronomical Society, 401, 2219

Breen S. L., Ellingsen S. P., Caswell J. L., Green J. A., Voronkov M. A., Fuller G. A., Quinn L. J., Avison A., 2012a, Monthly Notices of the Royal Astronomical Society, 421, 1703

Breen S. L., Ellingsen S. P., Caswell J. L., Green J. A., Voronkov M. A., Fuller G. A., Quinn L. J., Avison A., 2012b, Monthly Notices of the Royal Astronomical Society, 426, 2189

Breen S. L., Ellingsen S. P., Contreras Y., Green J. A., Caswell J. L., Stevens J. B., Dawson J. R., Voronkov M. A., 2013, Monthly Notices of the Royal Astronomical Society, 435, 524

Breen S. L., et al., 2014, Monthly Notices of the Royal Astronomical Society, 438, 3368

Breen S. L., et al., 2015, Monthly Notices of the Royal Astronomical Society, 450, 4109

Breen S. L., Ellingsen S. P., Caswell J. L., Green J. A., Voronkov M. A., Avison A., Fuller G. A., Quinn L. J., 2016, Monthly Notices of the Royal Astronomical Society, 459, 4066

Breen S. L., et al., 2018, Monthly Notices of the Royal Astronomical Society, 474, 3898

Butler M. J., Tan J. C., 2012, The Astrophysical Journal, 754, 5

Carey S. J., et al., 2009, Publications of the Astronomical Society of the Pacific, 121, 76

Caswell J. L., 2003, Monthly Notices of the Royal Astronomical Society, 341, 551

Caswell J. L., Vaile R. A., 1995, Monthly Notices of the Royal Astronomical Society, 273, 328

Caswell J. L., Kramer B. H., Reynolds J. E., 2009, Monthly Notices of the Royal Astronomical Society, 398, 528

Caswell J. L., et al., 2010, Monthly Notices of the Royal Astronomical Society, 404, 1029

Caswell J. L., et al., 2011, Monthly Notices of the Royal Astronomical Society, 417, 1964

Cesaroni R., 2005, Proceedings of the International Astronomical Union, 1, 59

Chanapote T., Asanok K., Dodson R., Rioja M., Green J. A., Hutawarakorn Kramer B., 2019, Monthly Notices of the Royal Astronomical Society, 482, 1670

Chen X., Gan C.-G., Ellingsen S. P., He J.-H., Shen Z.-Q., Titmarsh A., 2013, The Astrophysical Journal Supplement Series, 206, 9

Churchwell E., et al., 2009, Publications of the Astronomical Society of the Pacific, 121, 213

Cragg D. M., Johns K. P., Godfrey P. D., Brown R. D., 1992, Monthly Notices of the Royal Astronomical Society, 259, 203

Cragg D. M., Sobolev A. M., Ellingsen S. P., Caswell J. L., Godfrey P. D., Salii S. V., Dodson R. G., 2001, Monthly Notices of the Royal Astronomical Society, 323, 939

Cragg D. M., Sobolev A. M., Godfrey P. D., 2002, Monthly Notices of the Royal Astronomical Society, 331, 521

Cyganowski C. J., et al., 2008, The Astronomical Journal, 136, 2391
Cyganowski C. J., Brogan C. L., Hunter T. R., Churchwell E., 2009, The Astrophysical Journal, 702, 1615

Cyganowski C. J., Brogan C. L., Hunter T. R., Churchwell E., Zhang Q., 2011, The Astrophysical Journal, 729, 124

Dawson J. R., et al., 2014, Monthly Notices of the Royal Astronomical Society, 439, 1596

De Buizer J. M., Vacca W. D., 2010, The Astronomical Journal, 140, 196

Deacon R. M., Chapman J. M., Green A. J., Sevenster M. N., 2007, The Astrophysical Journal, 658, 1096

Dunham M. M., Crapsi A., Evans II N. J., Bourke T. L., Huard T. L., Myers P. C., Kauffmann J., 2008, The Astrophysical Journal Supplement Series, 179, 249

Egan M. P., Price S. D., Kraemer K. E., 2003, American Astronomical Society Meeting Abstracts, 203, 57.08

Elia D., et al., 2013, The Astrophysical Journal, 772, 45

Elia D., et al., 2017, Monthly Notices of the Royal Astronomical Society, 471, 100

Elitzur M., Hollenbach D. J., McKee C. F., 1989, The Astrophysical Journal, 346, 983

Ellingsen S. P., 2006, The Astrophysical Journal, 638, 241

Ellingsen S. P., 2007, Monthly Notices of the Royal Astronomical Society, 377, 571

Ellingsen S. P., Voronkov M. A., Cragg D. M., Sobolev A. M., Breen S. L., Godfrey P. D., 2007, Astrophysical Masers and their Environments, Proceedings of the International Astronomical Union, IAU Symposium, Volume 242, p. 213-217, 242, 213

Field D., Gray M. D., 1988, Monthly Notices of the Royal Astronomical Society, 234, 353

Fish V. L., 2007, The Astrophysical Journal, 669, L81

Forster J. R., Caswell J. L., 1989, Astronomy and Astrophysics, 213,339

Fujisawa K., et al., 2014, Publications of the Astronomical Society of Japan, 66, 31

Gallaway M., et al., 2013, Monthly Notices of the Royal Astronomical Society, 430, 808

Green J. A., McClure-Griffiths N. M., 2011, Monthly Notices of the Royal Astronomical Society, 417, 2500

Green J. A., et al., 2009, Monthly Notices of the Royal Astronomical Society, 392, 783

Green J. A., et al., 2010, Monthly Notices of the Royal Astronomical Society, 409, 913

Green J. A., et al., 2012, Monthly Notices of the Royal Astronomical Society, 420, 3108

Green J. A., et al., 2017, Monthly Notices of the Royal Astronomical Society, 469, 1383

Gutermuth R. A., Heyer M., 2015, The Astronomical Journal, 149,64

Guzmán A. E., Sanhueza P., Contreras Y., Smith H. A., Jackson J. M., Hoq S., Rathborne J. M., 2015, The Astrophysical Journal, 815, 130

Juvela M., et al., 2015, Astronomy \& Astrophysics, 584, A94

Juvela M., et al., 2018, Astronomy \& Astrophysics, 612, A71

Kauffmann J., Pillai T., 2010, The Astrophysical Journal, 723, L7

Kauffmann J., Pillai T., Shetty R., Myers P. C., Goodman A. A., 2010, The Astrophysical Journal, 712, 1137

Krumholz M. R., 2014, Physics Reports, 539, 49

Krumholz M. R., McKee C. F., 2008, Nature, 451, 1082

Kudritzki R. P., 2002, The Astrophysical Journal, 577, 389

Kurtz S., 2005, Proceedings of the International Astronomical Union, 1, 111

Lada C. J., Lada E. A., 2003, Annual Review of Astronomy and Astrophysics, 41, 57

Mainzer A., et al., 2011, The Astrophysical Journal, 731, 53

Minier V., Ellingsen S. P., Norris R. P., Booth R. S., 2003, Astronomy \& Astrophysics, 403, 1095 
Molinari S., et al., 2010a, Publications of the Astronomical Society of the Pacific, 122, 314

Molinari S., et al., 2010b, Astronomy \& Astrophysics, 518, L100

Molinari S., Schisano E., Faustini F., Pestalozzi M., Di Giorgio A. M., Liu S., 2011, Astronomy \& Astrophysics, 530, A133

Molinari S., et al., 2016a, Astronomy \& Astrophysics, 591, A149

Molinari S., Merello M., Elia D., Cesaroni R., Testi L., Robitaille T., 2016b, The Astrophysical Journal, 826, L8

Moscadelli L., Xu Y., Chen X., Moscadelli L., Xu Y., Chen X., 2010, ApJ, 716, 1356

Motte F., et al., 2010, Astronomy \& Astrophysics, 518, L77

Nguyên Luong Q., et al., 2011, Astronomy \& Astrophysics, 535, A76

Pandian J. D., Goldsmith P. F., Deshpande A. A., 2007, The Astrophysical Journal, 656, 255

Pandian J. D., Momjian E., Xu Y., Menten K. M., Goldsmith P. F., 2011, The Astrophysical Journal, 730, 55

Persi P., Tapia M., Roth M., Elia D., López-Vázquez J. A., 2016, Monthly Notices of the Royal Astronomical Society, 459, 1946

Preibisch T., Ossenkopf V., Yorke H. W., Henning T., 1993, Astronomy and Astrophysics, 279, 577

Qiao H.-H., et al., 2016, The Astrophysical Journal Supplement Series, 227, 26

Qiao H.-H., et al., 2018, The Astrophysical Journal Supplement Series, 239, 15

Reach W. T., et al., 2006, The Astronomical Journal, 131, 1479

Reid M. J., Dame T. M., Menten K. M., Brunthaler A., 2016, The Astrophysical Journal, 823, 77

Sanna A., Moscadelli L., Surcis G., van Langevelde H. J., Torstensson K. J. E., Sobolev A. M., 2017, Astronomy \& Astrophysics, 603, A94

Slysh V. I., Kalenskii S. V., Val'tts I. E., Otrupcek R., 1994, Monthly Notices of the Royal Astronomical Society, 268, 464

Sobolev A. M., Ostrovskii A. B., Kirsanova M. S., Shelemei O. V., Voronkov M. A., Malyshev A. V., 2005, Proceedings of the International Astronomical Union, 1, 174

Svoboda B. E., et al., 2016, The Astrophysical Journal, 822, 59

Tan J. C., et al., 2014, Massive Star Formation. University of Arizona Press

Titmarsh A. M., Ellingsen S. P., Breen S. L., Caswell J. L., Voronkov M. A., 2014, Monthly Notices of the Royal Astronomical Society, 443, 2923

Titmarsh A. M., Ellingsen S. P., Breen S. L., Caswell J. L., Voronkov M. A., 2016, Monthly Notices of the Royal Astronomical Society, 459, 157

Traficante A., et al., 2011, Monthly Notices of the Royal Astronomical Society, 416, 2932

Traficante A., Fuller G. A., Peretto N., Pineda J. E., Molinari S., 2015, Monthly Notices of the Royal Astronomical Society, 451,3089

Traficante A., Fuller G. A., Billot N., Duarte-Cabral A., Merello M., Molinari S., Peretto N., Schisano E., 2017, Monthly Notices of the Royal Astronomical Society, 470, 3882

Traficante A., Fuller G. A., Smith R. J., Billot N., Duarte-Cabral A., Peretto N., Molinari S., Pineda J. E., 2018, Monthly Notices of the Royal Astronomical Society, 473, 4975

Urquhart J. S., et al., 2015, Monthly Notices of the Royal Astronomical Society, 446, 3461

Urquhart J. S., et al., 2018, Monthly Notices of the Royal Astronomical Society, 473, 1059

Wall J. V., Jenkins C. R., 2012, Practical statistics for astronomers. Cambridge University Press

Walsh A. J., et al., 2011, Monthly Notices of the Royal Astronomical Society, 416, 1764

Walsh A. J., Purcell C. R., Longmore S. N., Breen S. L., Green J. A., Harvey-Smith L., Jordan C. H., Macpherson C., 2014, Monthly Notices of the Royal Astronomical Society, 442, 2240

Wright E. L., et al., 2010, The Astronomical Journal, 140, 1868
Zinnecker H., Yorke H. W., 2007, Annual Review of Astronomy and Astrophysics, 45, 481

van der Walt J., 2005, Monthly Notices of the Royal Astronomical Society, 360, 153

\section{APPENDIX A: PRINCIPAL COMPONENT ANALYSIS}

PCA is a statistical technique with which to reduce the dimensionality to only a few new dimensions. The new variables, the principal components, are defined along mutual trends in the $\mathrm{N}$-dimensional parameter space, where $\mathrm{N}$ is the original number of variables, each of which can be mapped back to the original physical variables (Wall \& Jenkins 2012). The direction of a principal component is identified as the direction of maximum variation in the N-D parameter space. For example, if three parameters are all mutually correlated with a linear relationship when plotted in $3 \mathrm{D}$, the first principal component will be in the direction along this correlation as the scatter, and therefore variation, about this line is minimised. Each subsequent principal component is identified in the remaining direction of maximum correlation after the previous trend has been removed, and therefore each accounts for a decreasing fraction of the total variance in the original parameter space. Only the first few components accounting for the majority of the original variance require further consideration as the components beyond this are only very weak correlations.

In addition to correlations, PCA is often applied to identify clustering in data sets with which to separate categories of objects. For our case of maser and non-maser objects, if the two samples cluster into separate regions in the parameter space defined by all of their properties, PCA will identify a principal component in the direction of separation to remove the variance caused by their separation. The two samples will display a maximum offset along this principal component, and physical trend responsible for the offset can be identified. For example, although we have already found that the maser objects are more massive, luminous and hotter than generic protostellar hosts on average, there remains a large overlap between the two types of object in these distributions. It is also not clear whether the same non-maser clumps overlap with the maser sample in all three properties. However, if each methanol maser host is simultaneously more massive, luminous and hotter than a general protostellar object, separation between maser and non-maser objects will be seen along a principal component in this direction.

In our analysis, the returned explained variance ratios (the fraction of total variance accounted for by each component) indicate that only the first two components should be considered further (0.51 and 0.32 respectively). Figure A1 shows the correlation of each principal component with the individual parameters. A single principal component might describe a mutual change in several properties with evolution of an object or with the size of an object. This decomposition of a principal component into the original parameters allows the direction of the new component to be interpreted as a physical trend. The first principal component identifies the strong correlation between $L_{70}, L_{250}$ and $L_{\mathrm{FIR}}$, but also the trend of increasing luminosity with the mutual increase of the mass and radius of a clump. The second component 


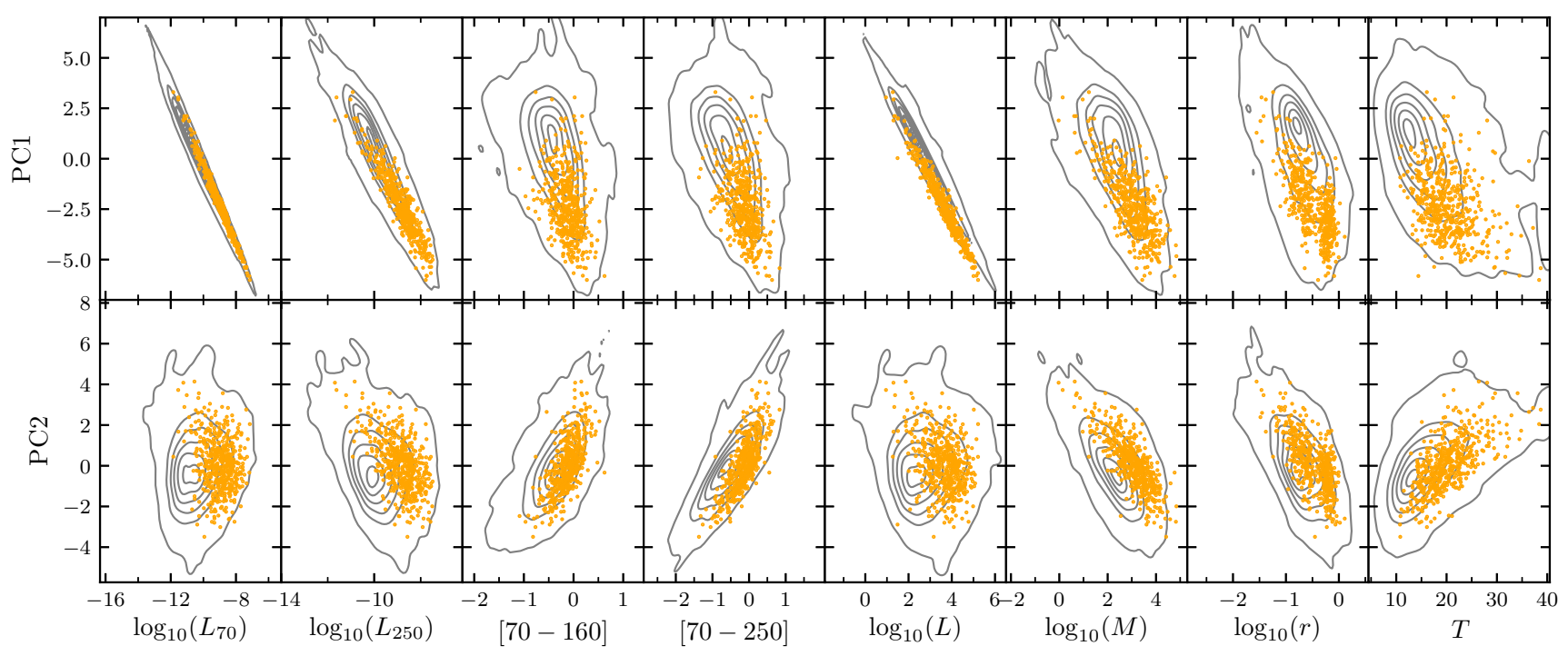

Figure A1. The decomposition of the first two principal components, PC1 (top row) and PC2(bottom row) into the physical source parameters used as input to the PCA. The correlation of each principal component with the source properties shows the direction of the principal component in the 8-dimensional infrared parameter space. The protostellar sources are shown as grey contours at density levels of $1 \%$ to $90 \%$ in intervals of $15 \%$, with the maser hosts marked in orange. The original infrared property units are $[\mathrm{L}: \mathrm{R}] \mathrm{L}_{\odot}, \mathrm{L}_{\odot}$, dimensionless, dimensionless, $\mathrm{L}_{\odot}, \mathrm{M}_{\odot}$, pc and $\mathrm{K}$ respectively, and the magnitudes of $\mathrm{PC} 1$ and $\mathrm{PC} 2$ are arbitrary.

is in the direction of increasing [70 - 160] and [70 - 250] colour with temperature alongside decreasing mass and radius. Although principal component analysis has identified that these trends are independent, the variance between the two samples is not sufficiently significant in comparison to the intra-sample variance in each property to identify a direction of separation between the two. PCA therefore selects the trends in parameter associated with evolutionary or scaling effects of the clumps. Non-linear clustering methods may be more appropriate for identifying small offsets, but these are much more challenging to map back to physical properties.

Making use of the independent clump property trends that have been identified through PCA, there are two possible explanations for the trend towards greater infrared colours seen in PC2. An increase in the amount of $70 \mu \mathrm{m}$ flux relative to longer wavelength fluxes for a source, indicated by an increase in [70-160] $\mu \mathrm{m}$ and [70-250] $\mu \mathrm{m}$ colours, may indicate an increase in the ability of short wavelength photons to escape from the inner regions of the clump, such as through holes in the envelope. The second possibility is that there is a greater amount of hot dust emitting at short wavelengths within the clump.

An enhancement in the ability of $70 \mu \mathrm{m}$ photons to escape from the inner regions should therefore correlate with an increase in the ability of photons to escape at $8 \mu \mathrm{m}$ if this is the underlying cause for this trend. In the case of removal of envelope material, the percentage of the inner surface area exposed by lines of sight with little or no intervening material determines the ability of photons to escape. We therefore expect a similar enhancement in the $70 \mu \mathrm{m}$ and $8 \mu \mathrm{m}$ emission through this effect and in such a case, the maser sources with $8 \mu \mathrm{m}$ counterparts would be expected to occupy the high IR colour regions of $\mathrm{PC} 2$, and therefore low-mass and radius regions. An increase in hot dust would also cause an increase in the amount of emission produced at $8 \mu \mathrm{m}$ by a protostar, although without the removal of envelope material, the $8 \mu \mathrm{m}$ emission is still much more strongly attenuated by the envelope material than emission at $70 \mu \mathrm{m}$. The correlation between increasing in $70 \mu \mathrm{m}$ emission and $8 \mu \mathrm{m}$ emission would be less pronounced in this case. When plotted the $8 \mu \mathrm{m}$ sources show no preferential location and are scattered throughout the entire regions occupied by the maser sources. This therefore supports the conclusion that an increase in the hot dust content of the clump is more likely responsible for this trend.

\section{APPENDIX B: CORRELATION OF MASER AND CLUMP LUMINOSITIES}

The figures to accompany Section 8.2 are presented here. Figure B1 displays the correlations between the luminosity of each $6.7 \mathrm{GHz}$ and secondary masers considered. Figure B2 shows the correlation and line of best fit between $6.7 \mathrm{GHz}$ and secondary $\mathrm{OH}$ maser luminosity.

This paper has been typeset from a $\mathrm{T}_{\mathrm{E} X} / \mathrm{LAT}_{\mathrm{E}} \mathrm{X}$ file prepared by the author. 


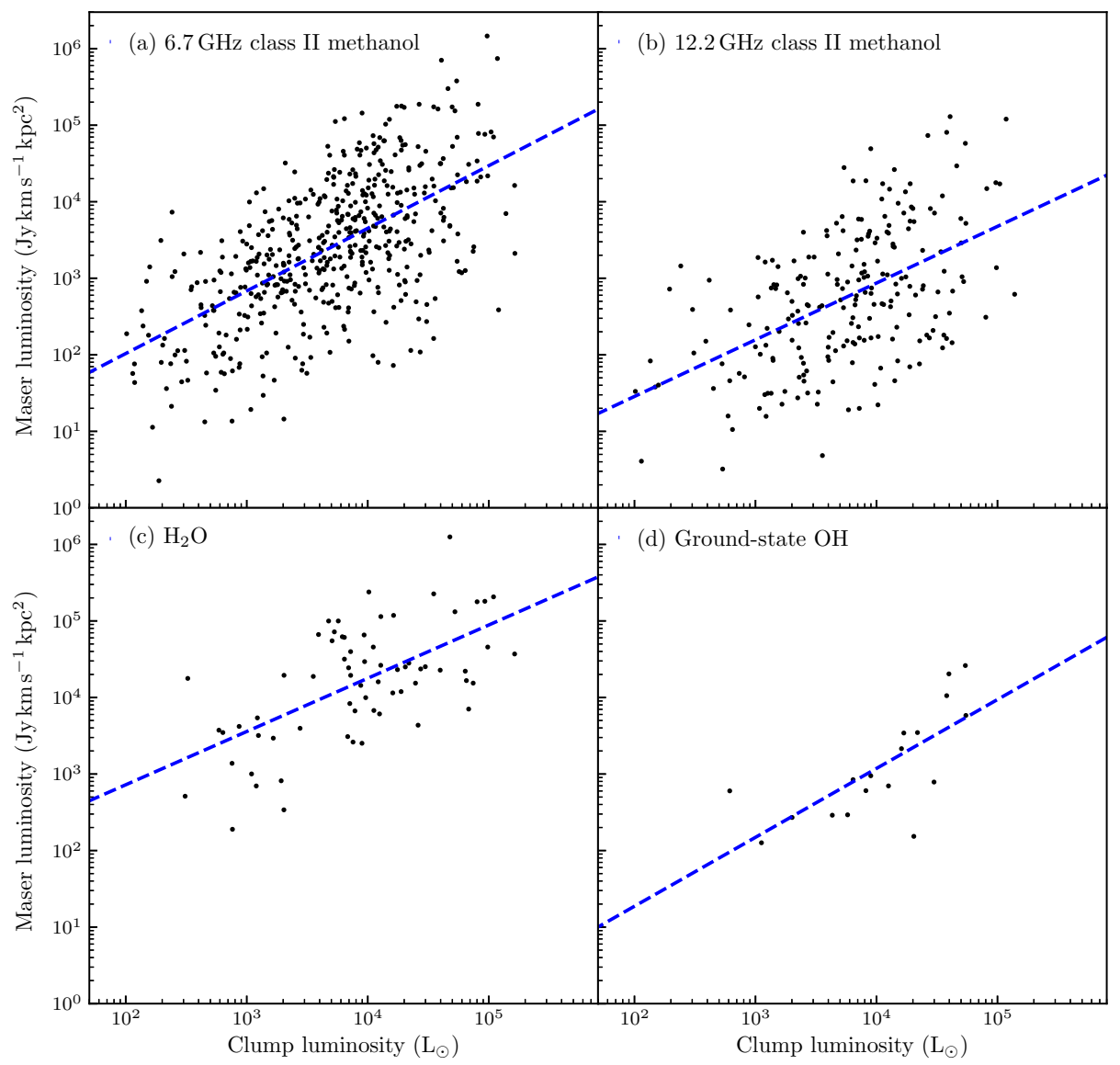

Figure B1. The correlation between integrated maser luminosity and host clump FIR luminosity for the $6.7 \mathrm{GHz}$ and other secondary masers within the clump. The panels show: (a) $6.7 \mathrm{GHz}$ luminosity and (b) $12.2 \mathrm{GHz}$ luminosity from the follow-up MMB study by Breen et al. (2012a,b, 2014, 2016), (c) the water maser luminosity from the study by Titmarsh et al. (2014, 2016) and (d) the OH maser luminosity from the SPLASH survey (Qiao et al. 2016, 2018), where the total integrated OH maser luminosity is calculated as the sum of the integrated luminosities of all individual $\mathrm{OH}$ masers in a clump. The lines of best fit are shown in blue dashed lines and the properties are given in Table 8 . 


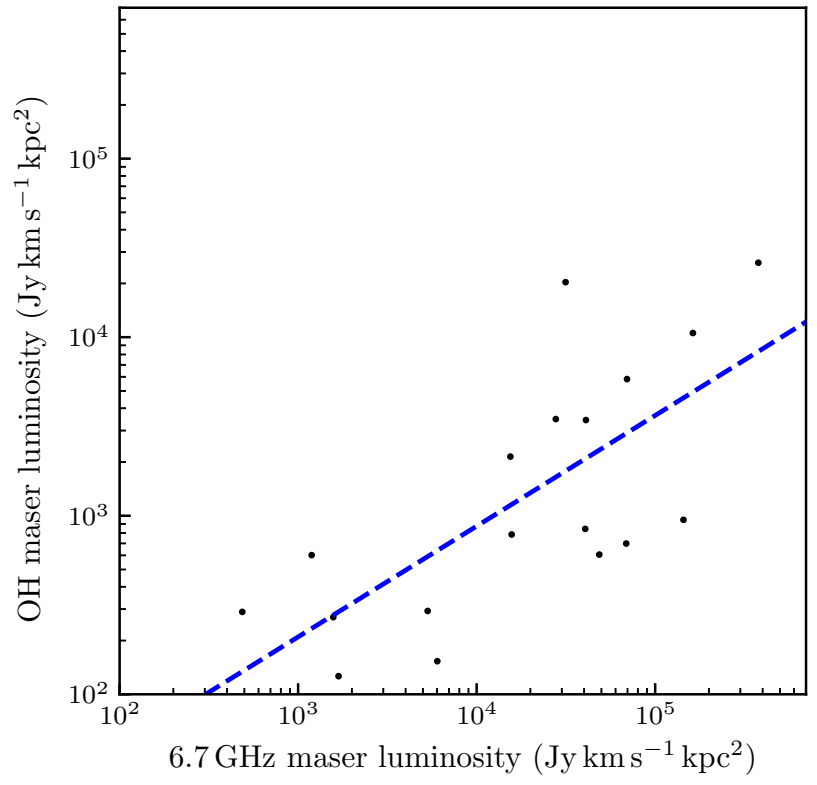

Figure B2. The correlation between integrated $6.7 \mathrm{GHz}$ maser luminosity and total integrated $\mathrm{OH}$ luminosity for the 18 clumps with a secondary $\mathrm{OH}$ maser and a reliable distance. The line of best fit (blue dashed) is given by $\log _{10}\left(L_{\mathrm{OH}}\right)=0.62 \log _{10}\left(L_{6.7}\right)+$ 0.46 . 